\title{
Delta-Nabla Type Maximum Principles for Second-Order Dynamic Equations on Time Scales and Applications
}

\author{
Jiang Zhu and Dongmei Liu \\ School of Mathematics and Statistics, Jiangsu Normal University, Xuzhou 221116, China \\ Correspondence should be addressed to Jiang Zhu; jzhuccy@163.com
}

Received 6 February 2014; Accepted 6 April 2014; Published 11 May 2014

Academic Editor: Dragoş-Pătru Covei

Copyright ( 2014 J. Zhu and D. Liu. This is an open access article distributed under the Creative Commons Attribution License, which permits unrestricted use, distribution, and reproduction in any medium, provided the original work is properly cited.

\begin{abstract}
Some delta-nabla type maximum principles for second-order dynamic equations on time scales are proved. By using these maximum principles, the uniqueness theorems of the solutions, the approximation theorems of the solutions, the existence theorem, and construction techniques of the lower and upper solutions for second-order linear and nonlinear initial value problems and boundary value problems on time scales are proved, the oscillation of second-order mixed delat-nabla differential equations is discussed and, some maximum principles for second order mixed forward and backward difference dynamic system are proved.
\end{abstract}

\section{Introduction}

Maximum principles are a well known tool for studying differential equations, which can be used to receive prior information about solutions of differential inequalities and to obtain lower and upper solutions of differential equations and so on. Maximum principles include continuous maximum principles and discrete maximum principles. It is well known that there are many results and applications for continuous and discrete maximum principles. For example, about these theories and applications, we can refer to [1-15] and the references therein. On the other hand, Hilger [16] established the theory of time scales calculus to unify the continuous and discrete calculus in 1990. After that, ordinary dynamic equations and partial dynamic equations on time scales have been extensively studied by some authors. For example, about these, we can refer to [17-23] and the references therein. However, the study on the maximum principles on time scales is very little, about these, we can refer to Stehik and Thompson's recent works [24, 25].

Inspired by the above works, we will be devoted to study delta-nabla type maximum principles for second-order dynamic equations on one-dimensional time scales and the applications of these maximum principles.

This paper is organized as follows. In Section 2, we state and prove some basic notations and results on time scales. In Section 3, we will first prove some delta-nabla type maximum principles for second-order dynamic equations on time scales; then, by using these maximum principles, we get some maximum principles for second-order mixed forward and backward difference dynamic system and discuss the oscillation of second-order mixed delta-nabla differential equations. In Section 4, we apply the maximum principles proved in Section 3 to obtain uniqueness of the solutions, the approximating techniques of the solutions, the existence theorem, and construction techniques of the lower and upper solutions for second-order linear initial value problems. In Section 5, we apply the maximum principles proved in Section 3 to obtain uniqueness of the solutions, the approximating techniques of the solutions, the existence theorem, and construction techniques of the lower and upper solutions for second-order linear boundary value problems. Finally, in Section 6, we extended the results of linear operator established in Sections 4 and 5 to nonlinear operators.

\section{Preliminaries}

Definition 1 (see [22]). A time scale $\mathbb{T}$ is a nonempty closed subset of the real numbers. Throughout this paper, $\mathbb{T}$ denotes a time scale.

Definition 2 (see [22]). Let $\mathbb{T}$ be a time scale. For $t \in \mathbb{T}$ one defines the forward jump operator $\sigma: \mathbb{T} \rightarrow \mathbb{T}$ by 
$\sigma(t):=\inf \{s \in \mathbb{T}: s>t\}$, while the backward jump operator $\rho: \mathbb{T} \rightarrow \mathbb{T}$ is defined by $\rho(t):=\sup \{s \in \mathbb{T}: s<t\}$. If $\sigma(t)>t$, one says that $t$ is right-scattered, while if $\rho(t)<t$, we say that $t$ is left-scattered. Points that are right-scattered and left-scattered at the same time are called isolated. Also, if $t<\sup \mathbb{T}$ and $\sigma(t)=t$, then $t$ is called right-dense, and if $t>\inf \mathbb{T}$ and $\rho(t)=t$, then $t$ is called left-dense. Finally, the graininess function $\mu(t): \mathbb{T} \rightarrow[0, \infty)$ is defined by

$$
\mu(t):=\sigma(t)-t
$$

Definition 3 (see [22]). If $\mathbb{T}$ has a left-scattered maximum $M$, then one defines $\mathbb{T}^{k}=\mathbb{T}-\{M\}$; otherwise $\mathbb{T}^{k}=\mathbb{T}$. Assume $f: \mathbb{T} \rightarrow R$ is a function and let $t \in \mathbb{T}^{k}$. Then one defines $f^{\Delta}(t)$ to be the number (provided it exists) with the property that, given any $\varepsilon>0$, there is a neighborhood $U$ of $t$ (i.e., $U=(t-\delta, t+\delta) \cap \mathbb{T}$ for some $\delta>0)$ such that

$$
\begin{aligned}
& \left|[f(\sigma(t))-f(s)]-f^{\Delta}(t)[\sigma(t)-s]\right| \\
& \quad \leq \varepsilon|\sigma(t)-s| \quad \forall s \in U .
\end{aligned}
$$

We call $f^{\Delta}(t)$ the delta derivative of $f$ at $t$.

Definition 4 (see [22]). If $\mathbb{T}$ has a right-scattered minimum $m$, then one defines $\mathbb{T}_{k}=\mathbb{T}-\{m\}$; otherwise $\mathbb{T}_{k}=\mathbb{T}$. The backwards graininess function $v(t): \mathbb{T} \rightarrow[0, \infty)$ is defined by

$$
\nu(t):=t-\rho(t)
$$

Assume $f: \mathbb{T} \rightarrow R$ is a function and let $t \in \mathbb{T}_{k}$. Then we define $f^{\nabla}(t)$ to be the number (provided it exists) with the property that, given any $\varepsilon>0$, there is a neighborhood $U$ of $t$ (i.e., $U=(t-\delta, t+\delta) \cap \mathbb{\mathbb { T }}$ for some $\delta>0)$ such that

$$
\begin{aligned}
& \left|[f(\rho(t))-f(s)]-f^{\nabla}(t)[\rho(t)-s]\right| \\
& \quad \leq \varepsilon|\rho(t)-s| \quad \forall s \in U .
\end{aligned}
$$

We call $f^{\nabla}(t)$ the nabla derivative of $f$ at $t$. Define the second derivative by $u^{\Delta \nabla}=\left(u^{\Delta}\right)^{\nabla}$.

Definition 5 (see [21]). Let $f: \mathbb{T} \rightarrow \mathbb{R}$. Define and denote $f \in C_{\mathrm{rd}}(\mathbb{T} ; \mathbb{R})$ as right-dense continuous if for each $t \in \mathbb{T}$

$\lim _{s \rightarrow t^{+}} f(s)=f(t), \quad$ if $t \in \mathbb{T}$ is right-dense,

$\lim _{s \rightarrow t^{-}} f(s)$ exists and is finite if $t \in \mathbb{T}$ is left-dense.

Definition 6 (see[21]). Let $f: \mathbb{T} \rightarrow \mathbb{R}$. Define and denote $f \in C_{\mathrm{ld}}(\mathbb{T} ; \mathbb{R})$ as left-dense continuous if for each $t \in \mathbb{T}$

$\lim _{s \rightarrow t^{-}} f(s)=f(t), \quad$ if $t \in \mathbb{T}$ is left-dense,

$\lim _{s \rightarrow t^{+}} f(s)$ exists and is finite if $t \in \mathbb{T}$ is right-dense.

Theorem 7 (see [21]). Assume that $f: f: \mathbb{T} \rightarrow \mathbb{R}$ and let $t \in \mathbb{T}^{k}$. (i) If $f$ is $\Delta$-differentiable at then $f$ is continuous at $t$.

(ii) If $f$ is continuous at $t$ and $t$ is right-scattered then $f$ is $\Delta$-differentiable at $t$ with

$$
f^{\Delta}(t)=\frac{f(\sigma(t))-f(t)}{\sigma(t)-t} .
$$

(iii) Ift is right-dense, then $f$ is differentiable at $t$ if and only if the limit

$$
\lim _{s \rightarrow t} \frac{f(t)-f(s)}{t-s}
$$

exists as a finite number. In this case

$$
f^{\Delta}(t)=\lim _{s \rightarrow t} \frac{f(t)-f(s)}{t-s} .
$$

(iv) If $f$ is $\Delta$-differentiable at $t$, then

$$
f(\sigma(t))=f(t)+\mu(t) f^{\Delta}(t) .
$$

Theorem 8 (see [22]). Assume that $f: f: \mathbb{T} \rightarrow \mathbb{R}$ and let $t \in \mathbb{T}_{k}$.

(i) If $f$ is nabla differentiable at $t$ then $f$ is continuous at $t$.

(ii) If $f$ is continuous at $t$ and $t$ is left-scattered then $f$ is nabla differentiable at $t$ with

$$
f^{\nabla}(t)=\frac{f(\rho(t))-f(t)}{\rho(t)-t} .
$$

(iii) Ift is left-dense, then $f$ is nabla differentiable at $t$ if and only if the limit

$$
\lim _{s \rightarrow t} \frac{f(t)-f(s)}{t-s}
$$

exists as a finite number. In this case

$$
f^{\nabla}(t)=\lim _{s \rightarrow t} \frac{f(t)-f(s)}{t-s} .
$$

(iv) If $f$ is nabla differentiable at $t$, then

$$
f(\rho(t))=f(t)-v(t) f^{\nabla}(t) .
$$

Theorem 9 (see [22]). If $f: \mathbb{T} \rightarrow \mathbb{R}$ is $\Delta$-differentiable and $f^{\Delta}$ is right-dense continuous on $\mathbb{T}^{k}$, then $f$ is $\nabla$-differentiable, and

$$
f^{\nabla}(t)= \begin{cases}f^{\Delta}(\rho(t)) & t \in \mathbb{T}_{A} \\ \lim _{s \rightarrow t^{-}} f^{\Delta}(s) & t \in A .\end{cases}
$$

If $g: \mathbb{T} \rightarrow \mathbb{R}$ is $\nabla$-differentiable and $g^{\nabla}$ is left-dense continuous on $\mathbb{T}_{k}$, then $f$ is $\Delta$-differentiable, and

$$
g^{\Delta}(t) \begin{cases}g^{\nabla}(\sigma(t)) & t \in \mathbb{T}_{B} \\ \lim _{s \rightarrow t^{+}} g^{\nabla}(s) & t \in B,\end{cases}
$$


where

$A:=\{t \in \mathbb{T}: t$ is left-dense and right-scattered $\}$,

$$
\mathbb{T}_{A}:=\mathbb{T} \backslash A,
$$

$B:=\{t \in \mathbb{T}: t$ is left-scattered and right-dense $\}$,

$$
\mathbb{T}_{B}:=\mathbb{T} \backslash B .
$$

Corollary 10 (see [22]). If $f: \mathbb{T} \rightarrow \mathbb{R}$ is $\Delta$-differentiable and $f^{\Delta}$ is continuous on $\mathbb{T}^{k}, g: \mathbb{T} \rightarrow \mathbb{R}$ is $\nabla$-differentiable, and $g^{\nabla}$ is continuous on $\mathbb{T}_{k}$, then

$$
\begin{array}{ll}
f^{\nabla}(t)=f^{\Delta}(\rho(t)) & \text { for } t \in \mathbb{T}_{k}, \\
g^{\Delta}(t)=g^{\nabla}(\sigma(t)) & \text { for } t \in \mathbb{T}^{k} .
\end{array}
$$

Theorem 11 (see [21]). Assume $f, g: \mathbb{T} \rightarrow \mathbb{R}$ are differentiable at $t \in \mathbb{T}^{k}$. Then

(i) the sum $f+g: \mathbb{T} \rightarrow \mathbb{R}$ is differentiable at $t$ with

$$
(f+g)^{\Delta}(t)=f^{\Delta}(t)+g^{\Delta}(t) ;
$$
with

(ii) for any constant $\alpha, \alpha f: \mathbb{T} \rightarrow \mathbb{R}$ is differentiable at $t$

$$
(\alpha f)^{\Delta}(t)=\alpha f^{\Delta}(t)
$$

(iii) the product $f g: \mathbb{T} \rightarrow \mathbb{R}$ is differentiable at $t$ with

$$
\begin{aligned}
(f g)^{\Delta}(t) & =f^{\Delta}(t) g(t)+f(\sigma(t)) g^{\Delta}(t) \\
& =f(t) g^{\Delta}(t)+f^{\Delta}(t) g(\sigma(t)) ;
\end{aligned}
$$

(iv) if $g(t) g(\sigma(t)) \neq 0$, then $\mathrm{f} / \mathrm{g}$ is differentiable at $t$ with

$$
\left(\frac{f}{g}\right)^{\Delta}(t)=\frac{f^{\Delta}(t) g(t)-f(t) g^{\Delta}(t)}{g(t) g(\sigma(t))} .
$$

Theorem 12 (see [22]). Assume $f, g: \mathbb{T} \rightarrow \mathbb{R}$ are nabla differentiable at $t \in \mathbb{T}_{k}$. Then

(i) the sum $f+g: \mathbb{T} \rightarrow \mathbb{R}$ is nabla differentiable at $t$ with

$$
(f+g)^{\nabla}(t)=f^{\nabla}(t)+g^{\nabla}(t)
$$

(ii) for any constant $\alpha, \alpha f: \mathbb{T} \rightarrow \mathbb{R}$ is nabla differentiable at $t$ with

$$
(\alpha f)^{\nabla}(t)=\alpha f^{\nabla}(t)
$$
with

(iii) the product $f g: \mathbb{T} \rightarrow \mathbb{R}$ is nabla differentiable at $t$

$$
\begin{aligned}
(f g)^{\nabla}(t) & =f^{\nabla}(t) g(t)+f(\rho(t)) g^{\nabla}(t) \\
& =f(t) g^{\nabla}(t)+f^{\nabla}(t) g(\rho(t)) ;
\end{aligned}
$$
with

(iv) if $g(t) g(\rho(t)) \neq 0$, then $\mathrm{f} / \mathrm{g}$ is nabla differentiable at $t$

$$
\left(\frac{f}{g}\right)^{\nabla}(t)=\frac{f^{\nabla}(t) g(t)-f(t) g^{\nabla}(t)}{g(t) g(\rho(t))}
$$

Theorem 13 (see [22]). If $f, f^{\Delta}$, and $f^{\nabla}$ are continuous, then

$$
\begin{aligned}
\text { (i) }\left[\int_{a}^{t} f(t, s) \Delta s\right]^{\Delta} & =\int_{a}^{t} f^{\Delta}(t, s) \Delta s+f(\sigma(t), t) ; \\
\text { (ii) }\left[\int_{a}^{t} f(t, s) \Delta s\right]^{\nabla} & =\int_{a}^{t} f^{\nabla}(t, s) \Delta s+f(\rho(t), \rho(t)) ; \\
\text { (iii) }\left[\int_{a}^{t} f(t, s) \nabla s\right]^{\Delta} & =\int_{a}^{t} f^{\Delta}(t, s) \nabla s+f(\sigma(t), \sigma(t)) ; \\
\text { (iv) }\left[\int_{a}^{t} f(t, s) \nabla s\right]^{\nabla} & =\int_{a}^{t} f^{\nabla}(t, s) \nabla s+f(\rho(t), t) .
\end{aligned}
$$

Definition 14 (see [21]). One says that a function $p: \mathbb{T} \rightarrow \mathbb{R}$ is regressive provided $1+\mu(t) p(t) \neq 0$ for all $t \in \mathbb{T}^{k}$ holds. The set of all regressive and rd-continuous functions $f: \mathbb{T} \rightarrow \mathbb{R}$ will be denoted by $\mathfrak{R}=\mathfrak{R}(\mathbb{T})=\mathfrak{R}(\mathbb{T}, \mathbb{R})$.

Definition 15 (see [21]). One defines $\xi_{h}(z)=(1 / h) \log (1+$ $z h)\left(\xi_{h}: \mathbb{C}_{h} \rightarrow \mathbb{Z}_{h}\right)$, where $h>0$. If $p \in \mathfrak{R}$, then one defines the exponential function by

$$
e_{p}(t, s)=\exp \left(\int_{s}^{t} \xi_{\mu(\tau)}(p(\tau)) \Delta \tau\right) \quad \text { for } s, t \in \mathbb{T} \text {. }
$$

If $p \in \Re$, then the first-order linear dynamic equation

$$
y^{\Delta}=p(t) y
$$

is called regressive.

Theorem 16 (see [21]). Suppose (28) is regressive and fix $t_{0} \in$ $\mathbb{T}$. Then $e_{p}\left(\cdot, t_{0}\right)$ is a solution of the initial value problem

$$
y^{\Delta}=p(t) y, \quad y\left(t_{0}\right)=1
$$

on $\mathbb{T}$.

Theorem 17 (see [21]). Suppose (28) is regressive; then the only solution of $(29)$ is given by $e_{p}\left(\cdot, t_{0}\right)$.

Theorem 18 (see [21]). If $p \in \mathfrak{R}$, then

(i) $e_{0}(t, s)=1$ and $e_{p}(t, t)=1$;

(ii) $e_{p}(\sigma(t), s)=(1+\mu(t) p(t)) e_{p}(t, s)$;

(iii) $e_{p}(t, s)=1 / e_{p}(s, t)$;

(iv) $\left(1 / e_{p}(\cdot, s)\right)^{\Delta}=-p(t) /\left(e_{p}^{\sigma}(\cdot, s)\right.$.

According to the above theorems and definitions, we can obtain the following corollary.

Corollary 19. Suppose (28) is regressive and fix $x_{0} \in \mathbb{T}_{k}^{k}$, and if one chooses $p(t)=\alpha$, where $\alpha$ is a positive constant, then the following equality holds on $\mathbb{T}_{k}^{k}$.
(a) $e_{\alpha}^{\nabla}\left(x, x_{0}\right)=\alpha e_{\alpha}\left(\rho(x), x_{0}\right)=\alpha \cdot\left(e_{\alpha}\left(x, x_{0}\right) /(1+\alpha \nu(x))\right)$.
(b) $e_{\alpha}^{\Delta \nabla}\left(x, x_{0}\right)=\alpha^{2} \cdot\left(e_{\alpha}\left(x, x_{0}\right) /(1+\alpha \nu(x))\right)$.

Proof. (a) Since

$$
\begin{gathered}
e_{\alpha}^{\nabla}\left(x, x_{0}\right)=e_{\alpha}^{\Delta}\left(\rho(x), x_{0}\right)=\alpha e_{\alpha}\left(\rho(x), x_{0}\right), \\
e_{\alpha}\left(\rho(x), x_{0}\right)=e_{\alpha}\left(x, x_{0}\right)-\nu(x) e_{\alpha}^{\nabla}\left(x, x_{0}\right),
\end{gathered}
$$


we have

$$
e_{\alpha}\left(\rho(x), x_{0}\right)=\frac{e_{\alpha}\left(x, x_{0}\right)}{1+\alpha \nu(x)},
$$

and thus

$$
e_{\alpha}^{\nabla}\left(x, x_{0}\right)=\alpha \cdot \frac{e_{\alpha}\left(x, x_{0}\right)}{1+\alpha \nu(x)} .
$$

(b) Obviously,

$$
\begin{aligned}
e_{\alpha}^{\Delta \nabla}\left(x, x_{0}\right) & =\left(\alpha \cdot e_{\alpha}\left(x, x_{0}\right)\right)^{\nabla} \\
& =\alpha^{2} e_{\alpha}\left(\rho(x), x_{0}\right) \\
& =\alpha^{2} \cdot \frac{e_{\alpha}\left(x, x_{0}\right)}{1+\alpha \nu(x)} .
\end{aligned}
$$

Definition 20 (see [22]). One defines $\widehat{\xi}_{h}(z)=-(1 / h) \log (1-$ $z h)\left(\widehat{\xi}_{h}: \mathbb{C}_{h} \rightarrow \mathbb{Z}_{h}\right)$, where $h>0$. If $p \in \mathfrak{R}_{v}$, then one defines the exponential function by

$$
\widehat{e}_{p}(t, s)=\exp \left(\int_{s}^{t} \widehat{\xi}_{\nu(\tau)}(p(\tau)) \nabla \tau\right) \quad \text { for } s, t \in \mathbb{T} .
$$

If $p \in \mathfrak{R}_{v}$, then the first-order linear dynamic equation

$$
y^{\nabla}=p(t) y
$$

is called regressive.

Theorem 21 (see [22]). Suppose (35) is regressive and fix $t_{0} \in$ $\mathbb{T}$. Then $\widehat{e}_{p}\left(\cdot, t_{0}\right)$ is a solution of the initial value problem

$$
y^{\nabla}=p(t) y, \quad y\left(t_{0}\right)=1
$$

on $\mathbb{T}$.

Theorem 22 (see [22]). Suppose (35) is regressive; then the only solution of (36) is given by $\widehat{e}_{p}\left(\cdot, t_{0}\right)$.

Theorem 23 (see [22]). If $p \in \mathfrak{R}_{v}$, then

(i) $\hat{e}_{0}(t, s)=1$ and $\widehat{e}_{p}(t, t)=1$;

(ii) $\widehat{e}_{p}(\rho(t), s)=(1-v(t) p(t)) \widehat{e}_{p}(t, s)$;

(iii) $\widehat{e}_{p}(t, s)=1 / \widehat{e}_{p}(s, t)$;

(iv) $\widehat{e}_{p}(t, u) \widehat{e}_{p}(u, s)=\widehat{e}_{p}(t, s)$;

(v) $\left(1 / \widehat{e}_{p}(\cdot, s)\right)^{\nabla}=-p(t) / e_{p}^{\rho}(\cdot, s)$.

Definition 24 (see [22]). One defines the set $\mathfrak{R}_{v}^{+}$of all positively $\nu$-regressive elements of $\Re_{v}$ by

$$
\mathfrak{R}_{v}^{+}=\left\{p \in \mathfrak{R}_{v}:(1-v(t) p(t))>0 \forall t \in \mathbb{T}\right\} .
$$

Corollary 25 (see [22]). If $p \in \mathfrak{R}_{v}^{+}$and $t_{0} \in \mathbb{T}$, then $\widehat{e}_{p}\left(t, t_{0}\right)>0$.
According to the above theorems and definitions, we can obtain the following corollary.

Corollary 26. Suppose (35) is regressive and fix $x_{0} \in \mathbb{T}_{k}^{k}$, and if one chooses $p(t)=\alpha$, where $\alpha$ is a negative constant, then the following equality holds on $\mathbb{T}_{k}^{k}$.

(a) $\widehat{e}_{\alpha}^{\Delta}\left(x, x_{0}\right)=\alpha \widehat{e}_{\alpha}\left(\sigma(x), x_{0}\right)=\alpha\left(\widehat{e}_{\alpha}\left(x, x_{0}\right) /(1-\alpha \mu(x))\right.$.

(b) $\hat{e}_{\alpha}^{\nabla}\left(x_{0}, x\right)=-\alpha \widehat{e}_{\alpha}\left(x_{0}, \rho(x)\right)=-\alpha\left(\widehat{e}_{\alpha}\left(x_{0}, x\right) /(1-\right.$ $\alpha \nu(x)))$.

(c) $\hat{e}_{\alpha}^{\Delta}\left(x_{0}, x\right)=-\alpha \cdot \hat{e}_{\alpha}\left(x_{0}, x\right)$.

(d) $\hat{e}_{\alpha}^{\Delta \nabla}\left(x_{0}, x\right)=\alpha^{2} \widehat{e}_{\alpha}\left(x_{0}, \rho(x)\right)=\alpha^{2}\left(\widehat{e}_{\alpha}\left(x_{0}, x\right) /(1-\right.$ $\alpha \nu(x)))$.

Proof. (a) It is easy to see that

$$
\widehat{e}_{\alpha}^{\Delta}\left(x, x_{0}\right)=\widehat{e}_{\alpha}^{\nabla}\left(\sigma(x), x_{0}\right)=\alpha \widehat{e}_{\alpha}\left(\sigma(x), x_{0}\right),
$$

and we have

$$
\begin{aligned}
\widehat{e}_{\alpha}\left(\sigma(x), x_{0}\right) & =\widehat{e}_{\alpha}\left(x, x_{0}\right)+\mu(x) \widehat{e}_{\alpha}^{\Delta}\left(x, x_{0}\right) \\
& =\widehat{e}_{\alpha}\left(x, x_{0}\right)+\mu(x) \alpha \widehat{e}_{\alpha}\left(\sigma(x), x_{0}\right),
\end{aligned}
$$

which can obtain

$$
\widehat{e}_{\alpha}\left(\sigma(x), x_{0}\right)=\frac{\widehat{e}_{\alpha}\left(x, x_{0}\right)}{1-\alpha \mu(x)},
$$

and thus

$$
\widehat{e}_{\alpha}^{\Delta}\left(x, x_{0}\right)=\alpha \widehat{e}_{\alpha}\left(\sigma(x), x_{0}\right)=\alpha \frac{\widehat{e}_{\alpha}\left(x, x_{0}\right)}{1-\alpha \mu(x)} .
$$

(b) Obviously,

$$
\begin{aligned}
\widehat{e}_{\alpha}^{\nabla}\left(x_{0}, x\right) & =\left(\frac{1}{\widehat{e}_{\alpha}\left(x, x_{0}\right)}\right)^{\nabla}=\frac{-\alpha}{\hat{e}_{\alpha}\left(\rho(x), x_{0}\right)} \\
& =-\alpha \widehat{e}_{\alpha}\left(x_{0}, \rho(x)\right),
\end{aligned}
$$

and we have

$$
\begin{aligned}
\widehat{e}_{\alpha}\left(x_{0}, \rho(x)\right) & =\widehat{e}_{\alpha}\left(x_{0}, x\right)-v(x) \widehat{e}_{\alpha}^{\nabla}\left(x_{0}, x\right) \\
& =\widehat{e}_{\alpha}\left(x_{0}, x\right)-v(x)\left[-\alpha \widehat{e}_{\alpha}\left(x_{0}, \rho(x)\right)\right],
\end{aligned}
$$

which can obtain

$$
\widehat{e}_{\alpha}\left(x_{0}, \rho(x)\right)=\frac{\widehat{e}_{\alpha}\left(x_{0}, x\right)}{1-\alpha \nu(x)},
$$

and therefore, we get

$$
\widehat{e}_{\alpha}^{\nabla}\left(x_{0}, x\right)=-\alpha \frac{\widehat{e}_{\alpha}\left(x_{0}, x\right)}{1-\alpha \nu(x)} .
$$

(c) We have

$$
\begin{aligned}
\hat{e}_{\alpha}^{\Delta}\left(x_{0}, x\right) & =\left(\frac{1}{\widehat{e}_{\alpha}\left(x, x_{0}\right)}\right)^{\Delta} \\
& =\frac{-\widehat{e}_{\alpha}^{\Delta}\left(x, x_{0}\right)}{\widehat{e}_{\alpha}\left(\sigma(x), x_{0}\right) \widehat{e}_{\alpha}\left(x, x_{0}\right)},
\end{aligned}
$$


and then

$$
\widehat{e}_{\alpha}^{\Delta}\left(x_{0}, x\right)=\frac{-\alpha \widehat{e}_{\alpha}\left(\sigma(x), x_{0}\right)}{\widehat{e}_{\alpha}\left(\sigma(x), x_{0}\right) \widehat{e}_{\alpha}\left(x, x_{0}\right)}=-\alpha \widehat{e}_{\alpha}\left(x_{0}, x\right) .
$$

(d) Obviously,

$$
\begin{aligned}
\widehat{e}_{\alpha}^{\Delta \nabla}\left(x_{0}, x\right) & =\left(\widehat{e}_{\alpha}^{\Delta}\left(x_{0}, x\right)\right)^{\nabla} \\
& =\left(-\alpha \widehat{e}_{\alpha}\left(x_{0}, x\right)\right)^{\nabla} \\
& =\alpha^{2} \widehat{e}_{\alpha}\left(x_{0}, \rho(x)\right) .
\end{aligned}
$$

And hence, we get

$$
\widehat{e}_{\alpha}^{\Delta \nabla}\left(x_{0}, x\right)=\alpha^{2} \widehat{e}_{\alpha}\left(x_{0}, \rho(x)\right)=\alpha^{2} \frac{\widehat{e}_{\alpha}\left(x_{0}, x\right)}{1-\alpha \nu(x)} .
$$

Theorem 27 (see [22]). Let $f$ be a continuous function on $[a, b]_{\mathbb{T}}$, that is, delta differentiable on $[a, b)_{\mathbb{T}}$. Then $f$ is increasing, decreasing, nondecreasing, and nonincreasing on $[a, b)_{\mathbb{T}}$ if $f^{\Delta}>0, f^{\Delta}<0, f^{\Delta} \geq 0, f^{\Delta} \leq 0$ for all $t \in[a, b)_{\mathbb{T}}$, respectively.

Definition 28. One says that a function $f: \mathbb{T} \rightarrow \mathbb{R}$ is leftincreasing at $t_{0} \in \mathbb{T}_{k}$ provided

(i) if $t_{0}$ is left-scattered, then $f\left(t_{0}\right)>f\left(\rho\left(t_{0}\right)\right)$;

(ii) if $t_{0}$ is left-dense, then there is a neighbourhood $U$ of $t_{0}$ such that $f\left(t_{0}\right)>f(t)$ for all $t \in U$ with $t_{0}>t$.

Similarly, we say that $f$ is left-decreasing if in the above (i) $f\left(t_{0}\right)<f\left(\rho\left(t_{0}\right)\right)$ and in (ii) $f\left(t_{0}\right)<f(t)$.

Theorem 29. Suppose $f: \mathbb{T} \rightarrow \mathbb{R}$ is nabla differentiable at $t_{0} \in \mathbb{T}_{k}$. If $f^{\nabla}\left(t_{0}\right)>0$, then $f$ is left-increasing. If $f^{\nabla}\left(t_{0}\right)<0$, then $f$ is left-decreasing.

Proof. We only show $f^{\nabla}\left(t_{0}\right)>0$ as the second statement can be shown similarly. If $t_{0}$ is left-scattered, then

$$
f^{\nabla}\left(t_{0}\right)=\frac{f\left(\rho\left(t_{0}\right)\right)-f\left(t_{0}\right)}{\rho\left(t_{0}\right)-t_{0}}
$$

and hence $f\left(\rho\left(t_{0}\right)\right)<f\left(t_{0}\right)$; that is, $f$ is left-increasing. Let now $t_{0}$ be left-dense. Then

$$
f^{\nabla}\left(t_{0}\right)=\lim _{t \rightarrow t_{0}} \frac{f\left(t_{0}\right)-f(t)}{t_{0}-t}
$$

and therefore for $\varepsilon=f^{\nabla}\left(t_{0}\right)$ there is a neighbourhood $U$ of $t_{0}$ such that

$$
\left|\frac{f\left(t_{0}\right)-f(t)}{t_{0}-t}-f^{\nabla}\left(t_{0}\right)\right|<\varepsilon
$$

for all $t \in U$ with $t \neq t_{0}$. Hence

$$
0<\frac{f\left(t_{0}\right)-f(t)}{t_{0}-t}<2 f^{\nabla}\left(t_{0}\right)
$$

Therefore, $f(t)<f\left(t_{0}\right)$ for all $t \in U$ with $t<t_{0}$. Combining what we have proved, we can get that if $f^{\nabla}\left(t_{0}\right)>0$, then $f$ is left-increasing.

Definition 30. We say that a function $f: \mathbb{T} \rightarrow \mathbb{R}$ assumes its local left-minimum at $t_{0} \in \mathbb{T}_{k}$ provided

(i) if $t_{0}$ is left-scattered, then $f\left(t_{0}\right) \leq f\left(\rho\left(t_{0}\right)\right)$;

(ii) if $t_{0}$ is left-dense, then there is a neighbourhood $U$ of $t_{0}$ such that $f\left(t_{0}\right) \leq f(t)$ for all $t \in U$ with $t_{0}>t$.

Similarly, we say that $f$ assumes its local left-maximum if in the above (i) $f\left(t_{0}\right) \geq f\left(\rho\left(t_{0}\right)\right)$ and in (ii) $f\left(t_{0}\right) \geq f(t)$.

Theorem 31. Suppose $f: \mathbb{T} \rightarrow \mathbb{R}$ is nabla differentiable at $t_{0} \in \mathbb{T}_{k}$. If $f$ attains its local left-minimum at $t_{0}$, then $f^{\nabla}\left(t_{0}\right) \leq$ 0. If $f$ attains its local left-maximum at $t_{0}$, then $f^{\nabla}\left(t_{0}\right) \geq 0$.

Proof. Suppose that $f$ attains its local left-minimum at $t_{0}$. To show that $f^{\nabla}\left(t_{0}\right) \leq 0$, we assume the opposite, that is, $f^{\nabla}\left(t_{0}\right)>0$. Then $f$ is left-increasing by Theorem 29 , contrary to the assumption that $f$ attains its local left-minimum at $t_{0}$. Thus, we must have $f^{\nabla}\left(t_{0}\right) \leq 0$. The second statement can be shown similarly.

Theorem 32. Let $f$ be a continuous function on $[a, b]_{\mathbb{T}}$, that $i$, nabla differentiable on $(a, b]_{\mathbb{T}}$ (the differentiability at $b$ is understood as left-sided) and satisfies $f(a)=f(b)$. Then, there exists $\xi, \tau \in(a, b]_{\mathbb{T}}$ such that

$$
f^{\nabla}(\xi) \leq 0 \leq f^{\nabla}(\tau)
$$

Proof. Since $f$ is continuous function on $[a, b]_{\mathbb{T}}, f$ attains its minimum $m$ and its maximum $M$. Therefore, here exists $\xi, \tau \in[a, b]_{\mathbb{T}}$ such that $m=f(\xi), M=f(\tau)$. Since $f(a)=$ $f(b)$, we may assume that $\xi, \tau \in(a, b]_{\mathbb{T}}$. Clearly $f$ attains its local left-minimum at $\xi$ and its local left-maximum at $\tau$. Then, by Theorem 31 we have $f^{\nabla}(\xi) \leq 0$ and $f^{\nabla}(\tau) \geq 0$.

Theorem 33. Let $f$ be a continuous function on $[a, b]_{\mathbb{T}}$, that is, nabla differentiable on $(a, b]_{\mathbb{T}}$. Then $f$ is increasing, decreasing, nondecreasing, and nonincreasing on $(a, b]_{\mathbb{T}}$ if $f^{\nabla}>0, f^{\nabla}<0$, $f^{\nabla} \geq 0, f^{\nabla} \leq 0$ for all $t \in(a, b]_{\mathbb{T}}$, respectively.

Proof. Suppose the function $\varphi(t)$ defined on $[a, c]_{\mathbb{T}} \in[a, b]_{\mathbb{T}}$ by

$$
\varphi(t)=f(t)-f(a)-\frac{f(c)-f(a)}{c-a}(t-a) .
$$

Clearly $\varphi(t)$ is continuous on $[a, c]_{\mathbb{T}}$ and nabla differentiable on $(a, c]_{\mathbb{V}}$. Also $\varphi(a)=\varphi(c)=0$, and so

$$
\varphi^{\nabla}(\xi) \leq 0 \leq \varphi^{\nabla}(\tau)
$$

for some $\xi, \tau \in(a, c]_{\mathbb{T}}$ by Theorem 32 . Hence, taking into account that

$$
\varphi^{\nabla}(t)=f^{\nabla}(t)-\frac{f(c)-f(a)}{c-a},
$$


then we have

$$
f^{\nabla}(\xi) \leq \frac{f(c)-f(a)}{c-a} \leq f^{\nabla}(\tau)
$$

for some $\xi, \tau \in(a, b]_{\mathbb{T}}$.

If $f^{\nabla}>0, f^{\nabla}<0, f^{\nabla} \geq 0, f^{\nabla} \leq 0$ for all $t \in(a, c]_{\mathbb{T}}$, then $f(c)-f(a)>0, f(c)-f(a)<0, f(c)-f(a) \geq 0$, $f(c)-f(a) \leq 0$, respectively. Considering the arbitrary of $c$, we arrive at the statement of the theorem.

\section{Delta-Nabla Type Maximum Principles}

In this paper, we denote $\Lambda:=[a, b]_{\mathbb{V}}$ as an interval on time scales. We study those functions defined on $\Lambda$ which belong to $\mathscr{D}(\Lambda)$, where $\mathscr{D}(\Lambda)$ is the set of all functions $u: \Lambda \rightarrow$ $\mathbb{R}$, such that $u^{\Delta}$ is continuous on $[a, b)_{\mathbb{T}}, u^{\nabla}$ is continuous on $(a, b]_{\mathbb{T}}$, and $u^{\Delta \nabla}$ exists in $(a, b)_{\mathbb{T}}$.

First we give a necessary condition that $u(x) \in \mathscr{D}(\Lambda)$ attains its maximum at some point $x_{0} \in(a, b)_{\mathbb{T}}$.

Lemma 34. If $u(x) \in \mathscr{D}(\Lambda)$ attains a maximum at a point $x_{0} \in(a, b)_{\mathbb{T}}$, then

$$
\begin{aligned}
& u^{\Delta \nabla}\left(x_{0}\right) \leq 0, \\
& u^{\nabla}\left(x_{0}\right) \geq 0, \\
& u^{\Delta}\left(x_{0}\right) \leq 0 .
\end{aligned}
$$

The strict inequality in the last two inequalities can occur only at left-scattered points.

Proof. Let us divide our proof into three parts.

(i) If $x_{0}$ is left-scattered, then the maximality of $u$ at $x_{0}$ implies that $u^{\nabla}\left(x_{0}\right) \geq 0$ and $u^{\Delta}\left(x_{0}\right) \leq 0$ and consequently

$$
\begin{aligned}
u^{\Delta \nabla}\left(x_{0}\right) & =\frac{u^{\Delta}\left(x_{0}\right)-u^{\Delta}\left(\rho\left(x_{0}\right)\right)}{v\left(x_{0}\right)} \\
& =\frac{u^{\Delta}\left(x_{0}\right)-u^{\nabla}\left(x_{0}\right)}{v\left(x_{0}\right)} \leq 0 .
\end{aligned}
$$

(ii) If $x_{0}$ is left-dense and right-scattered, in this case, we have $u^{\Delta}\left(x_{0}\right) \leq 0$. If there is no positive number sequence $\left\{h_{n}\right\}$ such that $\lim _{n \rightarrow \infty} h_{n}=0$ and $u^{\Delta}\left(x_{0}-h_{n}\right) \geq 0$, then there exists a $\delta>0$ such that $u^{\Delta}(x)<0$ for each $x \in\left[x_{0}-\delta, x_{0}\right)_{\mathbb{T}}$; by Theorem 27, a contraction with $u$ attains its maximum at interior point $x_{0}$ of $(a, b)_{\mathbb{T}}$. Thus, there exists $\left\{h_{n}\right\}$ such that $\lim _{n \rightarrow \infty} h_{n}=0$ and $u^{\Delta}\left(x_{0}-h_{n}\right) \geq 0$. This yields

$$
u^{\Delta \nabla}\left(x_{0}\right)=\lim _{h \rightarrow 0} \frac{u^{\Delta}\left(x_{0}\right)-u^{\Delta}\left(x_{0}-h\right)}{h} \leq 0 .
$$

Furthermore, the continuity of the delta derivative $u^{\Delta}(x)$ implies that

$$
0 \leq \lim _{h \rightarrow 0} u^{\Delta}\left(x_{0}-h\right)=u^{\Delta}\left(x_{0}\right) \leq 0,
$$

and consequently $u^{\Delta}\left(x_{0}\right)=0$. Then by using Corollary 10 we have that

$$
u^{\nabla}\left(x_{0}\right)=u^{\Delta}\left(\rho\left(x_{0}\right)\right)=u^{\Delta}\left(x_{0}\right)=0 .
$$

(iii) If $x_{0}$ is left-dense and right-dense, in this case the maximality of $u$ at $x_{0}$ and standard continuous necessary conditions imply that

$$
u^{\Delta \nabla}\left(x_{0}\right) \leq 0, \quad u^{\nabla}\left(x_{0}\right)=u^{\Delta}\left(x_{0}\right)=0 .
$$

According to Lemma 34, we can obtain the first simple maximum principle for the time scale.

Corollary 35. Assuming that $u(x) \in \mathscr{D}(\Lambda)$, if $u^{\Delta \nabla}\left(x_{0}\right)>0$ at some point $x_{0} \in(a, b)_{\mathbb{T}}$, then $u$ cannot attain its maximum at $x_{0}$. Moreover, if $u^{\Delta \nabla}(x)>0$ in $(a, b)_{\mathbb{T}}$, then $u$ cannot attain its maximum in $(a, b)_{\mathbb{T}}$.

We give a variant of Corollary 35 where we weaken the condition $u^{\Delta \nabla}>0$.

Theorem 36. Let $u(x) \in \mathscr{D}(\Lambda)$. If $u^{\Delta \nabla}(x) \geq 0$ in $(a, b)_{\mathbb{T}}$, then $u$ cannot attain its maximum in $(a, b)_{\mathbb{T}}$, unless $u \equiv M$.

Proof. We suppose that the result is false. Then there are $x_{0}, x_{1} \in(a, b)_{\mathbb{T}}$, such that $u\left(x_{0}\right)=M$ and $u\left(x_{1}\right)<M$. Let us assume first that $x_{0}<x_{1}$ and let us define a function $z(x) \in \mathscr{D}(\Lambda)$ by

$$
z(x):=e_{\alpha}\left(x, x_{0}\right)-1,
$$

where $\alpha>0$ and $e_{\alpha}\left(x, x_{0}\right)$ is an exponential function on $\mathbb{T}$ (see Section 2), and then

$$
z(x) \begin{cases}>0, & x>x_{0} \\ =0, & x=x_{0} \\ <0, & x<x_{0} .\end{cases}
$$

Considering $\alpha>0$ and the positivity of $e_{\alpha}$, we obtain

$$
z^{\Delta \nabla}(x)=\frac{\alpha^{2} e_{\alpha}\left(x, x_{0}\right)}{1+\alpha \nu}>0 .
$$

Let us define a function $w(x) \in \mathscr{D}(\Lambda)$ by

$$
w(x):=u(x)+\varepsilon z(x),
$$

where $\varepsilon>0$ is chosen so that

$$
\varepsilon<\frac{M-u\left(x_{1}\right)}{z\left(x_{1}\right)} .
$$

Since $e_{\alpha}\left(a, x_{0}\right)<1$, we have

$$
\begin{aligned}
w(a) & =u(a)+\varepsilon z(a) \\
& <u(a) \\
& \leq M .
\end{aligned}
$$


Furthermore, the definition of $\varepsilon$ yields that

$$
\begin{aligned}
w\left(x_{1}\right) & =u\left(x_{1}\right)+\varepsilon z\left(x_{1}\right) \\
& <u\left(x_{1}\right)+M-u\left(x_{1}\right)=M .
\end{aligned}
$$

Finally, $e_{\alpha}\left(x_{0}, x_{0}\right)=1$ derives

$$
w\left(x_{0}\right)=M
$$

It shows that $w$ attains its maximum in $\left(a, x_{1}\right)_{\mathbb{T}}$.

However,

$$
w^{\Delta \nabla}=u^{\Delta \nabla}+\varepsilon z^{\Delta \nabla}>0
$$

which contradicts the statement of Corollary 35. If $x_{0}>x_{1}$,

$$
z(x):=\int_{x}^{b}\left(\int_{s}^{b} \nabla \tau\right) \Delta s-\int_{x_{0}}^{b}\left(\int_{s}^{b} \nabla \tau\right) \Delta s .
$$

Then we have that

$$
\begin{gathered}
z(x) \begin{cases}<0, & x>x_{0} \\
=0, & x=x_{0} \\
>0, & x<x_{0}\end{cases} \\
z^{\Delta \nabla}(x)=1>0 .
\end{gathered}
$$

Let us define a function $w(x) \in \mathscr{D}(\Lambda)$ by

$$
w(x):=u(x)+\varepsilon z(x),
$$

where $\varepsilon>0$ is chosen so that

$$
\varepsilon<\frac{M-u\left(x_{1}\right)}{z\left(x_{1}\right)} .
$$

Since $z(b):=-\int_{x_{0}}^{b}\left(\int_{s}^{b} \nabla \tau\right) \Delta s<0$, we have

$$
\begin{aligned}
w(b) & =u(b)+\varepsilon z(b) \\
& <u(b) \\
& \leq M .
\end{aligned}
$$

Furthermore, the definition of $\varepsilon$ yields that

$$
w\left(x_{1}\right)=u\left(x_{1}\right)+\varepsilon z\left(x_{1}\right)<u\left(x_{1}\right)+M-u\left(x_{1}\right)=M .
$$

Finally, since $z\left(x_{0}\right)=0$, we derive

$$
w\left(x_{0}\right)=M
$$

It shows that $w$ attains its maximum in $\left(x_{1}, b\right)_{\mathbb{T}}$.

However,

$$
w^{\Delta \nabla}=u^{\Delta \nabla}+\varepsilon z^{\Delta \nabla}>0
$$

which is a contradiction with Corollary 35 . The proof is completed.
As a natural extension of the above simple maximum principle, we consider the operator of the following type:

$$
L[u]:=u^{\nabla \Delta}+g_{1} u^{\Delta}+g_{2} u^{\nabla}
$$

By the above results, we can obtain Theorem 37.

Theorem 37. Assume that the functions $g_{1}, g_{2}:[a, b]_{\mathbb{T}} \rightarrow R$ satisfy

$$
\begin{aligned}
& 1+v g_{1} \geq 0, \quad \text { on }(a, b)_{\mathbb{T}}, \\
& -1+v g_{2} \leq 0, \quad \text { on }(a, b)_{\mathbb{T}} .
\end{aligned}
$$

Letting $u(x) \in \mathscr{D}(\Lambda)$, if $L[u]\left(x_{0}\right)>0$ at some point $x_{0} \in$ $(a, b)_{\mathbb{T}}$, then $u$ cannot attain its maximum $M$ at $x_{0}$. Moreover, if $L[u]:=u^{\Delta \nabla}+g_{1} u^{\Delta}+g_{2} u^{\nabla}>0$, for each $x \in(a, b)_{\mathbb{T}}$, then $u$ cannot attain its maximum in $(a, b)_{\mathbb{T}}$.

Proof. We suppose that $L[u]\left(x_{0}\right)>0$ at some point $x_{0} \in$ $(a, b)_{\mathbb{T}}$ and $u$ attains its maximum at a point $x_{0}$. We divide our proof into two parts.

(i) If $x_{0}$ is left-scattered, in this case, we have

$$
\begin{aligned}
u^{\Delta \nabla}\left(x_{0}\right) & =\frac{u^{\Delta}\left(x_{0}\right)-u^{\Delta}\left(\rho\left(x_{0}\right)\right)}{v\left(x_{0}\right)} \\
& =\frac{u^{\Delta}\left(x_{0}\right)-u^{\nabla}\left(x_{0}\right)}{v\left(x_{0}\right)} .
\end{aligned}
$$

Multiplying $L[u]\left(x_{0}\right)$ by $\nu\left(x_{0}\right)$, we obtain

$$
\begin{aligned}
v\left(x_{0}\right) L[u]\left(x_{0}\right) \\
=v\left(x_{0}\right)\left(u^{\Delta \nabla}\left(x_{0}\right)+g_{1} u^{\Delta}\left(x_{0}\right)+g_{2} u^{\nabla}\left(x_{0}\right)\right) \\
=\left(1+v\left(x_{0}\right) g_{1}\right) u^{\Delta}\left(x_{0}\right) \\
\quad+\left(-1+v\left(x_{0}\right) g_{2}\right) u^{\nabla}\left(x_{0}\right) \\
>0 .
\end{aligned}
$$

However, it follows from Lemma 34 and the conditions

$$
\begin{aligned}
& 1+v g_{1} \geq 0, \quad \text { on }(a, b)_{\mathbb{T}}, \\
& -1+v g_{2} \leq 0, \quad \text { on }(a, b)_{\mathbb{T}},
\end{aligned}
$$

that $v\left(x_{0}\right) L[u]\left(x_{0}\right) \leq 0$, which is a contradiction.

(ii) If $x_{0}$ is left-dense, then by Lemma 34 we know that

$$
u^{\Delta}\left(x_{0}\right)=u^{\nabla}\left(x_{0}\right)=0 .
$$

Therefore, $L[u]\left(x_{0}\right)$ reduces to

$$
L\left[u\left(x_{0}\right)\right]=u^{\Delta \nabla}\left(x_{0}\right)>0,
$$

which is a contradiction with Lemma 34. Combining the proof of (i) and (ii), we get that $u$ cannot attain its maximum at $x_{0}$. The proof is completed. 
Next, we weaken the condition

$$
L[u]:=u^{\Delta \nabla}+g_{1} u^{\Delta}+g_{2} u^{\nabla}>0
$$

to

$$
L[u]:=u^{\Delta \nabla}+g_{1} u^{\Delta}+g_{2} u^{\nabla} \geq 0 .
$$

Theorem 38. Assume that the functions $g_{1}, g_{2}:[a, b]_{\mathbb{T}} \rightarrow R$ satisfy

$$
\begin{aligned}
& 1+v g_{1}>0, \quad \text { on }(a, b)_{\mathbb{T}}, \\
& -1+v g_{2}<0, \quad \text { on }(a, b)_{\mathbb{T}},
\end{aligned}
$$

$\frac{g_{1}+g_{2}}{1+g_{1} v}$ is bounded on any closed subinterval of $(a, b)_{\mathbb{T}}$.

Let $u(x) \in \mathscr{D}(\Lambda)$ satisfy $L[u]:=u^{\Delta \nabla}+g_{1} u^{\Delta}+g_{2} u^{\nabla} \geq 0$, for each $x \in(a, b)_{\mathbb{T}}$. Then $u$ cannot attain its maximum in $(a, b)_{\mathbb{T}}$, unless $u \equiv M$.

Proof. Assume that $u$ attains its maximum at a point $x_{0}$ in $(a, b)_{\mathbb{T}}$ but does not identically equal $M$. That is, $u\left(x_{0}\right)=M$, and there exists $x_{p} \in(a, b)_{\mathbb{T}}$ such that $u\left(x_{p}\right)<M$. Let us assume first that $x_{0}<x_{p}$ and let us define a function $z(x) \in$ $\mathscr{D}(\Lambda)$ by

$$
z(x)=e_{\alpha}\left(x, x_{0}\right)-1
$$

Therefore, we have

$$
\begin{aligned}
L[z] & =z^{\Delta \nabla}+g_{1} z^{\Delta}+g_{2} z^{\nabla} \\
& =\left[\left(1+v g_{1}\right) \alpha^{2}+\alpha \cdot\left(g_{1}+g_{2}\right)\right] \frac{e_{\alpha}\left(x, x_{0}\right)}{1+\alpha \nu} \\
& =\alpha\left[\alpha+\frac{g_{1}+g_{2}}{1+v g_{1}}\right] \frac{e_{\alpha}\left(x, x_{0}\right)}{1+\alpha \nu}\left(1+v g_{1}\right) .
\end{aligned}
$$

Thus, by (93) we can take arbitrary $\alpha>0$, such that $\alpha>-\left(g_{1}+\right.$ $\left.g_{2}\right) /\left(1+g_{1} v\right)$ in $\left[a_{1}, x_{p}\right]_{\mathbb{T}}$, where $a_{1}=\sigma(a)$ if $\sigma(a)>a$ and $a_{1} \in\left(a, x_{0}\right)_{\mathbb{T}}$ if $\sigma(a)=a$. Then we have $L[z]>0$ in $\left[a_{1}, x_{p}\right]_{\mathbb{T}}$. Let us define a function $w(x) \in \mathscr{D}(\Lambda)$ by

$$
w(x):=u(x)+\varepsilon z(x),
$$

where $\varepsilon>0$ is chosen so that

$$
\varepsilon<\frac{M-u\left(x_{p}\right)}{z\left(x_{p}\right)} .
$$

If $a_{1}=\sigma(a)=x_{0}$, since $e_{\alpha}\left(a, x_{0}\right)<1$, we have that $z(a)<0$ and

$$
\begin{aligned}
w(a) & =u(a)+\varepsilon z(a) \\
& <u(a) \\
& \leq M
\end{aligned}
$$

Moreover, the definition of $\varepsilon$ yields that

$$
\begin{gathered}
w\left(x_{p}\right)=u\left(x_{p}\right)+\varepsilon z\left(x_{p}\right)<u\left(x_{p}\right) \\
+M-u\left(x_{p}\right)=M .
\end{gathered}
$$

Finally, $e_{\alpha}\left(x_{0}, x_{0}\right)=1$ implies that $w\left(x_{0}\right)=M$. It follows that $w$ has a maximum in $\left(a, x_{p}\right)_{\mathbb{T}}$. However,

$$
L[w]=L[u]+\varepsilon L[z]>0,
$$

which is a contradiction with Theorem 37. If $a_{1}<x_{0}$, then we have $w\left(a_{1}\right)<M$. It follows that $w$ has a maximum in $\left(a_{1}, x_{p}\right)_{\pi}$. This is again a contradiction with Theorem 37 . Thus, we have proved that if $x_{0} \in(a, b)_{\mathbb{T}}$ is a maximum point, then $u(x)=$ $M$ for any $x \geq x_{0}$. Let

$$
m=\inf \left\{x \geq a: u(t)=M, \forall t \in\left[x, x_{0}\right]_{\mathbb{T}}\right\} .
$$

From this, we obtain that $u(m)=M$ and $u^{\Delta}(m)=0$. Then we have that $x_{0} \geq m>x_{p}$ and $u(t)<M$ for any $x_{0} \in(a, m)_{\mathbb{T}}$. If $m$ is left-scattered, then

$$
\begin{gathered}
\rho(m)<m, \\
u^{\Delta}(\rho(m))=\frac{u(m)-u(\rho(m))}{m-\rho(m)}>0 \\
u^{\Delta \nabla}(m)=\frac{u^{\Delta}(\rho(m))-u^{\Delta}(m)}{\rho(m)-m} \\
=\frac{u^{\Delta}(\rho(m))}{\rho(m)-m} .
\end{gathered}
$$

Since $L[u](m) \geq 0$, we multiply $L[u](m)$ by $v(m)$ and get that

$$
\begin{aligned}
0 & \leq L[u](m) \cdot v(m) \\
& =-u^{\Delta}(\rho(m))+u^{\nabla}(m) g_{2}(m) v(m) \\
& =-u^{\Delta}(\rho(m))+u^{\Delta}(\rho(m)) g_{2}(m) v(m) \\
& =u^{\Delta}(\rho(m))\left[g_{2}(m) v(m)-1\right]<0 .
\end{aligned}
$$

This is a contradiction. If $m$ is left-dense, let

$$
z(x)=e_{-\alpha}(x, m)-1
$$

where $\alpha>0$, such that $\alpha>\left(g_{1}+g_{2}\right) /\left(1+g_{1} \nu\right)$ in $\left[x_{p}, m\right]_{\mathbb{T}}$. We choose $d$ closely enough to $m$, such that $1-\alpha \mu>0,1-\alpha \nu>0$ on $[d, m]_{\mathbb{T}}$, and

$$
w(x):=u(x)+\varepsilon z(x),
$$

where $\varepsilon>0$ such that

$$
0<\varepsilon<\frac{M-u(d)}{z(d)} .
$$


Therefore, we have

$$
\begin{aligned}
L[z] & =z^{\Delta \nabla}+g_{1} z^{\Delta}+g_{2} z^{\nabla} \\
& =\left[\alpha^{2}\left(1+\nu g_{1}\right)-\alpha\left(g_{1}+g_{2}\right)\right] \frac{e_{-\alpha}(x, m)}{1-\alpha \nu} \\
& =\alpha\left[\alpha-\frac{g_{1}+g_{2}}{1+\nu g_{1}}\right] \frac{e_{-\alpha}(x, m)}{1-\alpha \nu}\left(1+\nu g_{1}\right)>0 .
\end{aligned}
$$

Thus, $L[w]=L[u]+\varepsilon L[z]>0$ on $[d, m]_{\mathbb{V}}$. By Theorem 37 we know that $w$ cannot attain its maximum in $(d, m)_{\mathbb{T}}$. Note that

$$
\begin{aligned}
w(d) & =u(d)+\varepsilon z(d) \\
& <u(d)+M-u(d)=M .
\end{aligned}
$$

We get that $w(m)=u(m)=M$ is the maximum of $w$ on $[d, m]_{\mathbb{T}}$. Since $u(x)=M$ for any $x \geq m$ and $z(x)$ is increasing for $x \geq m$, we have that $w^{\Delta}(m) \geq 0$; however, we also have that

$$
\begin{aligned}
w^{\Delta}(m) & =u^{\Delta}(m)+\varepsilon z^{\Delta}(m) \\
& =\varepsilon z^{\Delta}(m)=-\alpha \varepsilon<0 .
\end{aligned}
$$

This is a contradiction. The proof is completed.

In Theorem 38, if we take $\mathbb{T}=\mathbb{R}$, we have the following corollary which is the result that appeared in [3].

Corollary 39. Assuming that the function $g:[a, b] \rightarrow \mathbb{R}$ is bounded on any closed subinterval of $(a, b)$, if $u(x) \in \mathscr{D}(\Lambda)$ satisfies $(L+h)[u]:=u^{\prime \prime}+g u^{\prime \prime} \geq 0$ in $(a, b)$, then $u$ cannot attain its maximum $M$ in $(a, b)$, unless $u \equiv M$.

In Theorem 38, if we take $\mathbb{T}=\mathbb{Z}$, where $\mathbb{Z}$ is the set of all integral numbers, we can obtain the following new maximum principle for second-order mixed $\Delta$ and $\nabla$ difference dynamic system.

Corollary 40. Assume that the functions $g_{1}$ and $g_{2}$ : $[a, b]_{\mathbb{Z}} \rightarrow \mathbb{R}$ satisfy

$$
1+g_{1}(k)>0, \quad 1-g_{2}(k)>0, \quad \forall k \in(a, b)_{\mathbb{Z}}
$$

and let $u(x) \in \mathscr{D}(\Lambda)$; if

$$
\begin{aligned}
& \nabla \Delta u(k)+g_{1}(k) \Delta u(k) \\
&+g_{2}(k) \nabla u(k) \geq 0, \quad \forall k \in(a, b)_{\mathbb{Z}},
\end{aligned}
$$

then $u$ cannot attain its maximum $M$ in $(a, b)_{\mathbb{Z}}$, unless $u \equiv M$.

To show that conditions (91), (92), and (93) are necessary for the validity of our results, we give the following examples.

Example 41. Let $\mathbb{T}=\left\{q^{n}: n \in \mathbb{Z}\right\} \cup\{0\}$, where $\mathbb{Z}$ is the set of all integral numbers and $q>1$, and $u$ is defined by

$$
u(x)= \begin{cases}x, & \text { if } x \leq q^{8} \\ q^{8}, & \text { if } x>q^{8}\end{cases}
$$

Then

$$
\begin{gathered}
u^{\Delta}(x)= \begin{cases}1, & \text { if } x<q^{8} ; \\
0, & \text { if } x>q^{8}, \\
0, & \text { if } x=q^{8},\end{cases} \\
u^{\nabla}(x)= \begin{cases}1, & \text { if } x<q^{8} ; \\
0, & \text { if } x>q^{8}, \\
1, & \text { if } x=q^{8},\end{cases} \\
u^{\Delta \nabla}(x)= \begin{cases}0, & \text { if } x<q^{8} ; \\
0, & \text { if } x>q^{8}, \\
\frac{1}{q^{7}(1-q)}, & \text { if } x=q^{8} .\end{cases}
\end{gathered}
$$

Letting $g_{1}(x) \equiv 1, g_{2}(x) \equiv 1 / \nu(x), x \in[a, b]_{\mathbb{T}}=\left[1, q^{9}\right]_{\mathbb{T}}$, then

$$
\begin{gathered}
u^{\Delta \nabla}(x)+g_{1}(x) u^{\Delta}(x)+g_{2}(x) u^{\nabla}(x) \\
=u^{\Delta \nabla}(x)+u^{\Delta}(x)+u^{\nabla}(x) \geq 0
\end{gathered}
$$

$1+v(x) g_{1}(x)=1+1>0,1-v(x) g_{2}(x)=1-1=0$ for any $x \in\left[1, q^{9}\right]_{\mathbb{T}}$, and

$$
\frac{g_{1}(x)+g_{2}(x)}{1+g_{1}(x) \nu(x)}=\frac{1+(1 / \nu(x))}{1+\nu(x)}=\frac{1}{\nu(x)}
$$

is bounded on any closed subinterval of $\left[1, q^{9}\right]_{\mathbb{T}}$. Thus, conditions (91) and (93) hold, but (92) does not hold. The conclusion of Theorem 38 also does not hold, since $u$ attains its maximum $q^{8}$ in $\left(1, q^{9}\right)_{\mathbb{Z}}$, but $u$ is not constant.

Example 42. Let $\mathbb{T}=\left\{q^{n}: n \in \mathbb{Z}\right\} \cup\{0\}$, where $\mathbb{Z}$ is the set of all integral numbers and $q>1$, and $u$ is defined by

$$
u(x)= \begin{cases}q^{5}, & \text { if } x \leq q^{5} \\ 2 q^{5}-x, & \text { if } x>q^{5}\end{cases}
$$

Then

$$
\begin{gathered}
u^{\Delta}(x)= \begin{cases}0, & \text { if } x<q^{5} ; \\
-1, & \text { if } x>q^{5}, \\
-1, & \text { if } x=q^{5},\end{cases} \\
u^{\nabla}(x)= \begin{cases}0, & \text { if } x<q^{5} ; \\
-1, & \text { if } x>q^{5}, \\
0, & \text { if } x=q^{5},\end{cases} \\
u^{\Delta \nabla}(x)= \begin{cases}0, & \text { if } x<q^{5} ; \\
0, & \text { if } x>q^{5}, \\
\frac{1}{q^{4}(1-q)}, & \text { if } x=q^{5} .\end{cases}
\end{gathered}
$$

Letting $g_{1}(x)=g_{2}(x) \equiv-1 / \nu(x),[a, b]_{\mathbb{T}}=\left[1, q^{7}\right]_{\mathbb{T}}$, then

$$
\begin{gathered}
u^{\Delta \nabla}(x)+g_{1}(x) u^{\Delta}(x)+g_{2}(x) u^{\nabla}(x) \\
=u^{\Delta \nabla}(x)-u^{\Delta}(x)-u^{\nabla}(x) \geq 0
\end{gathered}
$$


$1+\nu(x) g_{1}(x)=1-1=0,1-v(x) g_{2}(x)=1+1>0$ for any $x \in\left[1, q^{7}\right]_{\mathbb{T}}$, and $\left(g_{1}+g_{2}\right) /\left(1+g_{1} v\right)=1$ is bounded on any closed subinterval of $\left[1, q^{7}\right]_{\mathbb{T}}$. Thus, condition (92) holds, but (91) does not hold; thus (93) does hold. The conclusion of Theorem 38 also does not hold, since $u$ attains its maximum $q^{5}$ in $\left(1, q^{7}\right)_{\mathbb{Z}}$, but $u$ is not constant.

Example 43. Let $\mathbb{T}=\{1 / n: n \in \mathbb{Z}\} \cup\{0\}, u(x)=-e_{\alpha}(x, 0)$, $[a, b]_{\mathbb{T}}=[-1 / 2,1 / 2]_{\mathbb{T}}$, and $2<\alpha<3$. Then

$$
\begin{aligned}
u^{\Delta}(x) & =-\alpha e_{\alpha}(x, 0), \\
u^{\nabla}(x) & =-\frac{\alpha}{1+\alpha \nu(x)} e_{\alpha}(x, 0), \\
u^{\Delta \nabla}(x) & =-\frac{\alpha^{2}}{1+\alpha \nu(x)} e_{\alpha}(x, 0) .
\end{aligned}
$$

Let

$$
\begin{gathered}
g_{1}(x)= \begin{cases}\frac{-\alpha}{|x|}, & \text { if } x=\frac{1}{n} \\
0 & \text { if } x=0\end{cases} \\
g_{2}(x)=-\alpha,
\end{gathered}
$$

then

$$
\begin{gathered}
u^{\Delta \nabla}(x)+g_{1}(x) u^{\Delta}(x)+g_{2}(x) u^{\nabla}(x) \\
=\left[-\frac{\alpha^{2}}{1+\alpha \nu(x)}-\alpha g_{1}(x)+\frac{\alpha^{2}}{1+\alpha \nu(x)}\right] \\
\times e_{\alpha}(x, 0) \\
\geq 0, \\
1+v(x) g_{1}(x)= \begin{cases}1+\frac{-\alpha}{|n+1|}>0, & \text { if } x=\frac{1}{n}, \\
1>0 & \text { if } x=0,\end{cases} \\
1-v(x) g_{2}(x)= \begin{cases}1+\frac{\alpha}{n(n+1)}>0, & \text { if } x=\frac{1}{n}, \\
1>0 & \text { if } x=0,\end{cases} \\
\frac{g_{1}(x)+g_{2}(x)}{1+v(x) g_{1}(x)}= \begin{cases}\frac{-\alpha n-\alpha}{1+(-\alpha /|n+1|)}, & \text { if } x=\frac{1}{n}, \\
-\alpha & \text { if } x=0 .\end{cases}
\end{gathered}
$$

These show that conditions (91) and (92) hold, but (93) does not hold on $[-1 / 2,1 / 2]_{\mathbb{T}}$. The conclusion of Theorem 38 also does not hold on $[-1 / 2,1 / 2]_{\mathbb{T}}$, since $u$ attains its maximum -1 in $(-1 / 2,1 / 2)_{\mathbb{T}}$, but $u$ is not constant.

Now, we establish a generalized maximum principle.

Theorem 44. Assume that the functions $g_{1}$ and $g_{2}:[a, b]_{\mathbb{T}} \rightarrow \mathbb{R}$ satisfy (91), (92), and let $u(x) \in \mathscr{D}(\Lambda)$ and

$$
(L+h)[u]:=u^{\Delta \nabla}+g_{1} u^{\Delta}+g_{2} u^{\nabla}+h u,
$$

where $h(x) \leq 0$. If $(L+h)[u]\left(x_{0}\right)>0$ at some point $x_{0} \in$ $(a, b)_{\mathbb{T}}$, then $u$ cannot attain its maximum $M$ at $x_{0}$. Moreover, if $(L+h)[u](x)>0$, for each $x \in(a, b)_{\mathbb{T}}$, then $u$ cannot attain its maximum in $(a, b)_{\mathbb{T}}$.

Proof. Assume that $u$ attains its nonnegative maximum $M$ at a point $x_{0}$ in $(a, b)_{\mathbb{T}}$ and $(L+h)[u]\left(x_{0}\right)>0$. If $x_{0}$ is leftscattered, then by Lemma 34 , we have that

$$
\begin{aligned}
\rho\left(x_{0}\right)< & x_{0}, \\
u^{\nabla}\left(x_{0}\right) & =u^{\Delta}\left(\rho\left(x_{0}\right)\right) \\
& =\frac{u\left(x_{0}\right)-u\left(\rho\left(x_{0}\right)\right)}{x_{0}-\rho\left(x_{0}\right)} \geq 0, \\
u^{\Delta \nabla}\left(x_{0}\right) & =\frac{u^{\Delta}\left(\rho\left(x_{0}\right)\right)-u^{\Delta}\left(x_{0}\right)}{\rho\left(x_{0}\right)-x_{0}}, \\
u^{\Delta}\left(x_{0}\right) & \leq 0
\end{aligned}
$$

and then

$$
\begin{aligned}
0< & (L+h)[u]\left(x_{0}\right) \cdot v\left(x_{0}\right) \\
= & -u^{\Delta}\left(\rho\left(x_{0}\right)\right)+u^{\Delta}\left(x_{0}\right)+u^{\Delta}\left(x_{0}\right) g_{1}\left(x_{0}\right) v\left(x_{0}\right) \\
& +u^{\nabla}\left(x_{0}\right) g_{2}\left(x_{0}\right) v\left(x_{0}\right)+h\left(x_{0}\right) u\left(x_{0}\right) v\left(x_{0}\right) \\
= & -u^{\nabla}\left(x_{0}\right)+u^{\Delta}\left(x_{0}\right)+u^{\Delta}\left(x_{0}\right) g_{1}\left(x_{0}\right) v\left(x_{0}\right) \\
& +u^{\nabla}\left(x_{0}\right) g_{2}\left(x_{0}\right) v\left(x_{0}\right)+h\left(x_{0}\right) u\left(x_{0}\right) v\left(x_{0}\right) \\
= & u^{\nabla}\left(x_{0}\right)\left[g_{2}\left(x_{0}\right) v\left(x_{0}\right)-1\right] \\
& +u^{\Delta}\left(x_{0}\right)\left[1+g_{1}\left(x_{0}\right) v\left(x_{0}\right)\right] \\
& +h\left(x_{0}\right) u\left(x_{0}\right) v\left(x_{0}\right) \leq 0 .
\end{aligned}
$$

This is a contradiction. If $x_{0}$ is left-dense, by Lemma 34 we have that $u^{\Delta}\left(x_{0}\right)=u^{\nabla}\left(x_{0}\right)=0$ and $u^{\Delta \nabla}\left(x_{0}\right) \leq 0$. Then

$$
(L+h)[u]\left(x_{0}\right)=u^{\Delta \nabla}\left(x_{0}\right)+h\left(x_{0}\right) u\left(x_{0}\right)>0,
$$

and $h\left(x_{0}\right) u\left(x_{0}\right) \leq 0$ imply that $u^{\Delta \nabla}\left(x_{0}\right)>0$. This is also a contradiction with Corollary 35 . Thus, we have that $u$ cannot attain its maximum $M$ at $x_{0}$. The proof is completed.

In Theorem 44 , if we take $\mathbb{T}=\mathbb{R}$, we have the following corollary which is an improvement for the result that appeared in [3].

Corollary 45. Let $g, h:[a, b] \rightarrow \mathbb{R}$ be functions, $h(x) \leq 0$ on $[a, b]$ and $(L+h)[u]:=u^{\prime \prime}+g u^{\prime}+h u$. If $(L+h)[u]\left(x_{0}\right)>0$ at some point $x_{0} \in(a, b)$, then $u$ cannot attain its maximum $M$ at $x_{0}$. Moreover, if $(L+h)[u](x)>0$, for each $x \in(a, b)$, then $u$ cannot attain its maximum in $(a, b)_{\mathbb{T}}$.

In Theorem 44 , if we take $\mathbb{T}=\mathbb{Z}$, where $\mathbb{Z}$ is the set of all integral numbers, we can obtain the following new maximum principle for second-order mixed $\Delta$ and $\nabla$ difference dynamic system. 
Corollary 46. Assume that the functions $h, g_{1}$, and $g_{2}$ : $[a, b]_{\mathbb{Z}} \rightarrow \mathbb{R}$ satisfy

$$
1+g_{1}(k)>0, \quad 1-g_{2}(k)>0, \quad \forall k \in(a, b)_{\mathbb{Z}}
$$

and $h(k) \leq 0$ on $[a, b]_{\mathbb{Z}}$. Let $u(x) \in \mathscr{D}(\Lambda)$. If for some $k \in$ $(a, b)_{\mathbb{Z}}$

$$
\nabla \Delta u(k)+g_{1}(k) \Delta u(k)+g_{2}(k) \nabla u(k)+h(k) u(k)>0,
$$

then $u$ cannot attain its maximum $M$ at $k$. If

$$
\begin{gathered}
\nabla \Delta u(k)+g_{1}(k) \Delta u(k)+g_{2}(k) \nabla u(k) \\
+h(k) u(k)>0, \quad \forall k \in(a, b)_{\mathbb{Z}},
\end{gathered}
$$

then $u$ cannot attain its maximum $M$ in $(a, b)_{\mathbb{Z}}$.

Theorem 47. Assume that the functions $g_{1}$ and $g_{2}:[a, b]_{\mathbb{T}} \rightarrow$ $\mathbb{R}$ satisfy (91), (92), and (93), and let $u(x) \in \mathscr{D}(\Lambda)$ satisfy

$$
\begin{array}{r}
(L+h)[u]:=u^{\Delta \nabla}+g_{1} u^{\Delta}+g_{2} u^{\nabla}+h u \geq 0, \\
\text { for each } x \in(a, b)_{\mathbb{T}},
\end{array}
$$

where $h(x) \leq 0$ and

$$
\frac{h(x)}{1+\nu g_{1}} \text { is bounded on any closed subinterval of }(a, b)_{\mathbb{T}} \text {. }
$$

If $u$ attains a nonnegative maximum $M$ in $(a, b)_{\mathbb{T}}$, then $u \equiv M$.

Proof. Assume that $u$ attains its nonnegative maximum $M$ at a point $x_{0}$ in $(a, b)_{\mathbb{T}}$ but does not identically equal $M$. Thus, we can choose $x_{0}, x_{p} \in(a, b)_{\mathbb{T}}$, such that $u\left(x_{0}\right)=M, u\left(x_{p}\right)<M$. If $x_{p}>x_{0}$, we define a function $z(x) \in \mathscr{D}(\Lambda)$ by

$$
z(x)=e_{\alpha}\left(x, x_{0}\right)-1 .
$$

Then

$$
\begin{aligned}
(L+h)[z] & \\
= & z^{\Delta \nabla}+g_{1} z^{\Delta}+g_{2} z^{\nabla}+h z \\
= & {\left[\left(1+v g_{1}\right) \alpha^{2}+\alpha \cdot\left(g_{1}+g_{2}\right)+h(1+\alpha v(x))\right.} \\
& \left.\times\left(1-e_{\alpha}\left(x_{0}, x\right)\right)\right] \frac{e_{\alpha}\left(x, x_{0}\right)}{1+\alpha \nu} \\
= & {\left[\alpha^{2}+\alpha \cdot \frac{g_{1}+g_{2}}{1+v g_{1}}\right.} \\
& \left.+\frac{h(1+\alpha v(x))\left(1-e_{\alpha}\left(x_{0}, x\right)\right)}{1+v g_{1}}\right] \\
& \times \frac{e_{\alpha}\left(x, x_{0}\right)\left(1+v g_{1}\right)}{1+\alpha \nu} .
\end{aligned}
$$

It is similar to the proof of Theorem 38; we choose sufficiently larger $\alpha$ such that

$$
(L+h)[z]>0
$$

holds on $\left(a_{1}, x_{p}\right)_{\mathbb{T}}$, where $x_{0} \in\left(a_{1}, x_{p}\right)_{\mathbb{T}} \subset\left(a, x_{p}\right)_{\mathbb{T}}$. Let us define a function $w(x) \in \mathscr{D}(\Lambda)$ by

$$
w(x):=u(x)+\varepsilon z(x),
$$

where $\varepsilon>0$ is chosen so that

$$
\varepsilon<\frac{M-u\left(x_{p}\right)}{z\left(x_{p}\right)} .
$$

Since $e_{\alpha}\left(a, x_{0}\right)<1$, we have

$$
\begin{aligned}
w(a) & =u(a)+\varepsilon z(a) \\
& <u(a) \\
& \leq M
\end{aligned}
$$

Moreover, the definition of $\varepsilon$ yields that

$$
\begin{aligned}
w\left(x_{p}\right)= & u\left(x_{p}\right)+\varepsilon z\left(x_{p}\right)<u\left(x_{p}\right) \\
& +M-u\left(x_{p}\right)=M .
\end{aligned}
$$

Finally, $e_{\alpha}\left(x_{0}, x_{0}\right)=1$ implies that $w\left(x_{0}\right)=M$. It implies that $w$ has a maximum in $\left(a, x_{p}\right)_{\mathbb{T}}$. However,

$$
\begin{aligned}
L[w] & \geq(L+h)[w] \\
& =(L+h)[u]+\varepsilon(L+h)[z]>0
\end{aligned}
$$

holds on $\left(a_{1}, x_{p}\right)_{\mathbb{T}}$. This is a contradiction with Theorem 37. Thus, we have proved that if $x_{0} \in(a, b)_{\mathbb{T}}$ is a maximum point, then $u(x)=M$ for any $x \geq x_{0}$. Let

$$
m=\inf \left\{x \geq a: u(t)=M, \forall t \in\left[x, x_{0}\right]_{\mathbb{T}}\right\} .
$$

From this, we obtain that $u(m)=M$ and $u^{\Delta}(m)=0$. Then we have that $x_{0} \geq m>x_{p}$ and $u(t)<M$ for any $x_{0} \in$ $(a, m)_{\mathbb{T}}$. If $m$ is left-scattered, then it is similar to the proof of Theorem 38; we have that

$$
\rho(m)<m,
$$

$$
\begin{aligned}
u^{\Delta}(\rho(m)) & =\frac{u(m)-u(\rho(m))}{m-\rho(m)}>0 \\
u^{\Delta \nabla}(m) & =\frac{u^{\Delta}(\rho(m))-u^{\Delta}(m)}{\rho(m)-m} \\
& =\frac{u^{\Delta}(\rho(m))}{\rho(m)-m}
\end{aligned}
$$

$$
\begin{aligned}
0 \leq & L[u](m) \cdot v(m) \\
= & -u^{\Delta}(\rho(m))+u^{\nabla}(m) g_{2}(m) v(m) \\
& +h(m) u(m) v(m) \\
= & -u^{\Delta}(\rho(m))+u^{\Delta}(\rho(m)) g_{2}(m) v(m) \\
& +h(m) u(m) v(m) \\
= & u^{\Delta}(\rho(m))\left[g_{2}(m) v(m)-1\right] \\
& +h(m) u(m) v(m)<0 .
\end{aligned}
$$


This is a contradiction. If $m$ is left-dense, let

$$
z(x)=e_{-\alpha}(x, m)-1,
$$

where $\alpha>0$, such that

$$
\alpha>\frac{g_{1}+g_{2}}{1+v g_{1}}-\frac{h}{1+v g_{1}}
$$

in $\left[x_{p}, m\right]_{\mathbb{V}}$. We choose $d$ closely enough to $m$, such that $1-$ $\alpha \mu>0,1-\alpha \nu>0$ on $[d, m]_{\mathbb{T}}$ and

$$
w(x):=u(x)+\varepsilon z(x),
$$

where $\varepsilon>0$ such that

$$
0<\varepsilon<\frac{M-u(d)}{z(d)} .
$$

Therefore, we have

$$
\begin{aligned}
L[z]= & z^{\Delta \nabla}+g_{1} z^{\Delta}+g_{2} z^{\nabla}+h z \\
= & {\left[\alpha^{2}\left(1+\nu g_{1}\right)-\alpha\left(g_{1}+g_{2}\right)\right] } \\
& \quad \times \frac{e_{-\alpha}(x, m)}{1-\alpha \nu}+h\left(e_{-\alpha}(x, m)-1\right) \\
= & \alpha\left\{\alpha-\frac{g_{1}+g_{2}}{1+\nu g_{1}}+\frac{h}{1+\nu g_{1}}(1-\alpha \nu)\right. \\
& \left.\quad \times\left(1-\frac{1}{e_{-\alpha}(x, m)}\right)\right\} \frac{e_{-\alpha}(x, m)}{1-\alpha \nu}\left(1+v g_{1}\right)>0 .
\end{aligned}
$$

Thus, $L[w]=L[u]+\varepsilon L[z]>0$ on $[d, m]_{\mathbb{T}}$. By Theorem 38 we know that $w$ cannot attain its maximum in $(d, m)_{\mathbb{T}}$. Note that

$$
w(d)=u(d)+\varepsilon z(d)<u(d)+M-u(d)=M
$$

we get that $w(m)=u(m)=M$ is the maximum of $w$ on $[d, m]_{\mathbb{T}}$. This implies that $w^{\Delta}(m) \geq 0$; however, we also have that

$$
w^{\Delta}(m)=u^{\Delta}(m)+\varepsilon z^{\Delta}(m)=\varepsilon z^{\Delta}(m)=-\alpha \varepsilon<0 .
$$

This is a contradiction. The proof is completed.

Corollary 48. Assume that $h(x)$ is not always equal to 0 in Theorem 47; if $u$ attains its nonnegative maximum $M$ in $(a, b)_{\mathbb{T}}$, then the nonnegative maximum $M=0$. Especially, if $u(a) \leq 0, u(b) \leq 0$, then $u(x)<0$ for $x \in(a, b)_{\mathbb{T}}$, unless $u(x) \equiv 0$.

In Theorem 47, if we take $\mathbb{T}=\mathbb{R}$, we have the following corollary which is the result that appeared in [3].

Corollary 49. Assuming that the functions $g, h:[a, b] \rightarrow \mathbb{R}$ onin $(a, b)$ and $h(x) \leq 0$ on $[a, b]$, if $u(x) \in \mathscr{D}(\Lambda)$ satisfies $(L+h)[u]:=u^{\prime \prime}+g u^{\prime}+h u \geq 0$ in $(a, b)$, then $u$ cannot attain its maximum $M$ in $(a, b)$, unless $u \equiv M$.
In Theorem 47 , if we take $\mathbb{T}=\mathbb{Z}$, where $\mathbb{Z}$ is the set of all integral numbers, we can obtain the following new maximum principle for second-order mixed $\Delta$ and $\nabla$ difference dynamic system.

Corollary 50. Assume that the functions $h, g_{1}$, and $g_{2}$ : $[a, b]_{\mathbb{Z}} \rightarrow \mathbb{R}$ satisfy

$$
1+g_{1}(k)>0, \quad 1-g_{2}(k)>0, \quad \forall k \in(a, b)_{\mathbb{Z}}
$$

and $h(k) \leq 0$ on $[a, b]_{\mathbb{Z} .}$ If $u(x) \in \mathscr{D}(\Lambda)$ satisfies

$$
\begin{gathered}
\nabla \Delta u(k)+g_{1}(k) \Delta u(k)+g_{2}(k) \nabla u(k) \\
+h(k) u(k) \geq 0, \quad \forall k \in(a, b)_{\mathbb{Z}},
\end{gathered}
$$

then $u$ cannot attain its maximum $M$ in $(a, b)_{\mathbb{Z}}$, unless $u \equiv M$.

All of the above results investigate the behavior of functions inside the considered interval. Now, we will discuss the behavior of functions by providing the information about the boundary points.

Theorem 51. Let $g_{1}, g_{2}$, and $h$ satisfy (91), (92), and $h(x) \leq 0$. Assume that $u(x) \in \mathscr{D}(\Lambda)$ is not constant, such that

$$
(L+h)[u]:=u^{\Delta \nabla}+g_{1} u^{\Delta}+g_{2} u^{\nabla}+h u \geq 0,
$$

for each $x \in(a, b)_{\mathbb{T}}$, u has unilateral derivative at points of $a, b$, and (93), (130) hold on $(a, b)_{\mathbb{T}}$.

(1) If $u$ attains its nonnegative maximum at a point of $a$, then $u^{\Delta}(a)<0$;

(2) If $u$ attains its nonnegative maximum at a point of $b$, then $u^{\nabla}(b)>0$.

Proof. We suppose that $u$ attains its nonnegative maximum $M$ at $a$, that is, $u(a)=M$, and there exists a point $x_{0} \in[a, b]_{\mathbb{T}}$, such that $u\left(x_{0}\right)<M$; we define a function $z(x) \in \mathscr{D}(\Lambda)$ by

$$
z(x)=e_{\alpha}(x, a)-1,
$$

where $\alpha>0$. It is similar to the proof of Theorem 38; we can choose a larger enough $\alpha$, such that

$$
(L+h)[z]>0 .
$$

Moreover, we define a function $w(x) \in \mathscr{D}(\Lambda)$ by

$$
w(x)=u(x)+\varepsilon z(x),
$$

where

$$
0<\varepsilon<\frac{M-u\left(x_{0}\right)}{z\left(x_{0}\right)} .
$$

Thus

$$
(L+h)[w]>0
$$

and by using Theorem 47 to $w$ on $\left[a, x_{0}\right]_{\mathbb{T}}$, we get that $w$ attains its maximum at $a$ or $x_{0}$. Note that $w(a)=M>w\left(x_{0}\right)$, 
and thus $w$ attains its maximum at $a$. Therefore, unilateral derivative of $w(a)$ is not positive:

$$
w^{\Delta}(a)=u^{\Delta}(a)+\varepsilon z^{\Delta}(a) \leq 0 .
$$

However,

$$
z^{\Delta}(a)=\alpha>0
$$

and hence

$$
u^{\Delta}(a)<0
$$

If $u(b)=M$, we can prove $u^{\nabla}(b)>0$ as the similar way above. The proof is completed.

In Theorem 51, if we take $\mathbb{T}=\mathbb{R}$, we have the following corollary which is the result that appeared in [3].

Corollary 52. Assuming that the functions $g, h:[a, b] \rightarrow \mathbb{R}$ are bounded in $(a, b)$ and $h(x) \leq 0$ on $[a, b]$, if $u(x) \in \mathscr{D}(\Lambda)$ satisfies $(L+h)[u]:=u^{\prime \prime}+g u^{\prime}+h u \geq 0$ in $(a, b)$, and $u$ has unilateral derivative at points of $a, b$.

(1) If $u$ attains its nonnegative maximum at a point of $a$, then $u^{\prime}(a)<0$;

(2) If $u$ attains its nonnegative maximum at a point of $b$, then $u^{\prime}(b)>0$.

In Theorem 51 , if we take $\mathbb{T}=\mathbb{Z}$, where $\mathbb{Z}$ is the set of all integral numbers, we can obtain the following new maximum principle for second-order mixed $\Delta$ and $\nabla$ difference dynamic system.

Corollary 53. Assume that the functions $h, g_{1}$, and $g_{2}$ : $[a, b]_{\mathbb{Z}} \rightarrow \mathbb{R}$ satisfy

$$
1+g_{1}(k)>0, \quad 1-g_{2}(k)>0, \quad \forall k \in(a, b)_{\mathbb{Z}},
$$

and $h(k) \leq 0$ on $[a, b]_{\mathbb{Z}}$, and assume that $u(x) \in \mathscr{D}(\Lambda)$ satisfies

$$
\begin{gathered}
\nabla \Delta u(k)+g_{1}(k) \Delta u(k)+g_{2}(k) \nabla u(k) \\
+h(k) u(k) \geq 0, \quad \forall k \in(a, b)_{\mathbb{Z}} .
\end{gathered}
$$

(1) If $u$ attains its nonnegative maximum at a point of $a$, then $u(a+1)<u(a)$;

(2) If $u$ attains its nonnegative maximum at a point of $b$, then $u(b-1)>u(b)$.

Next, we consider that $(L+h)[u] \geq 0$, and $h(x)$ may take positive value. Let $g_{1}, g_{2}$, and $h(x)$ satisfy (91) and (92). Assume that we can find a function $w(x) \in \mathscr{D}(\Lambda)$ which satisfies

$$
\begin{array}{cl}
w(x)>0 & \text { for each } x \in \Lambda, \\
(L+h)[w] \leq 0 & \text { for each } x \in(a, b)_{\mathbb{V}}
\end{array}
$$

Then there exists a function $F$ which is predifferentiable with region of differentiation $\Lambda$ such that

$$
F^{\nabla}(x)=w(x) \quad \forall x \in \Lambda,
$$

and therefore, $w^{\sigma \nabla}(x)=F^{\Delta \nabla}(x)$ since $w^{\sigma}(x)=F^{\nabla}(\sigma(x))=$ $F^{\Delta}(x)$.

We define a new function $v(x) \in \mathscr{D}(\Lambda)$ by

$$
v(x)=\frac{u(x)}{w(x)},
$$

and then $v(x)$ satisfies

$$
\begin{gathered}
v^{\Delta \nabla}+\frac{F^{\Delta \nabla}+g_{1} w^{\sigma}}{w^{\sigma \rho}} v^{\Delta}+\frac{w^{\Delta \rho}+g_{2} w^{\rho}}{w^{\sigma \rho}} v^{\nabla} \\
+\frac{1}{w^{\sigma \rho}}(L+h)[w] v \geq 0 .
\end{gathered}
$$

Lemma 54. Assume that (91), (92), and (93) hold, and a function $w(x)$ satisfies (162). Then, the following inequalities hold in $(a, b)_{\mathbb{T}}$;

(a) $\left(1 / w^{\sigma \rho}\right)(L+h)[w] \leq 0$,

(b) $1+v\left(\left(F^{\Delta \nabla}+g_{1} w^{\sigma}\right) / w^{\sigma \rho}\right)>0$,

(c) $-1+v\left(\left(w^{\Delta \rho}+g_{2} w^{\rho}\right) / w^{\sigma \rho}\right)<0$.

Moreover, if $g_{1}, g_{2}, w^{\Delta \nabla}$, and $h(x)$ are bounded on $(a, b)_{\mathbb{T}}$, and there exist $t_{1}>0$, such that

$$
1+v g_{1} \geq t_{1}>0, \quad \text { on }(a, b)_{\mathbb{T}}
$$

then $\left(G_{1}+G_{2}\right) /\left(1+\nu G_{1}\right)$ and $H /\left(1+\nu G_{1}\right)$ are bounded on $(a, b)_{\mathbb{T}}$, where

$$
\begin{gathered}
G_{1}=\frac{F^{\Delta \nabla}+g_{1} w^{\sigma}}{w^{\sigma \rho}}, \quad G_{2}=\frac{w^{\Delta \rho}+g_{2} w^{\rho}}{w^{\sigma \rho}}, \\
H=\frac{1}{w^{\sigma \rho}}(L+h)[w] .
\end{gathered}
$$

Proof. (a) Since

$$
\begin{gathered}
(L+h)[w] \leq 0 \\
w(x)>0
\end{gathered}
$$

then

$$
\frac{1}{w^{\sigma \rho}}(L+h)[w] \leq 0 .
$$

If $\nu(x)=0$, then (b) and (c) are satisfied at $x$, and so we suppose that $\nu(x)>0$.

(b) It is easy to see that

$$
\begin{aligned}
\nu F^{\Delta \nabla}(x) & =\nu \frac{F^{\Delta}(x)-F^{\Delta}(\rho(x))}{\nu} \\
& =F^{\Delta}(x)-F^{\nabla}(x) \\
& =w^{\sigma}(x)-w(x) .
\end{aligned}
$$

Since $\nu(x)>0$, and hence

$$
\sigma(\rho(x))=x,
$$


then

$$
w(x)=w^{\sigma \rho}(x)
$$

and, therefore,

$$
\begin{aligned}
1+v & \frac{F^{\Delta \nabla}+g_{1} w^{\sigma}}{w^{\sigma \rho}} \\
& =1+\frac{v w^{\sigma \nabla}+\nu g_{1} w^{\sigma}}{w^{\sigma \rho}} \\
& =1+\frac{w^{\sigma}-w^{\sigma \rho}+v g_{1} w^{\sigma}}{w^{\sigma \rho}} \\
& =\left(1+v g_{1}\right) \frac{w^{\sigma}}{w^{\sigma \rho}}>0 .
\end{aligned}
$$

(c) Finally,

$$
v w^{\Delta \rho}=[x-\rho(x)] \frac{w^{\sigma}(\rho(x))-w(\rho(x))}{\sigma(\rho(x))-\rho(x)} .
$$

Since $v(x)>0$, and hence

$$
\sigma(\rho(x))=x
$$

thus

$$
\begin{aligned}
-1+ & \nu \frac{w^{\Delta \rho}+g_{2} w^{\rho}}{w^{\sigma \rho}} \\
& =\frac{-w^{\sigma \rho}+w^{\sigma \rho}-w^{\rho}+\nu g_{2} w^{\rho}}{w^{\sigma \rho}} \\
& =\left(-1+v g_{2}\right) \frac{w^{\rho}}{w^{\sigma \rho}} \\
& <0 .
\end{aligned}
$$

The boundness of $\left(G_{1}+G_{2}\right) /\left(1+\nu G_{1}\right)$ and $H /\left(1+\nu G_{1}\right)$ can be deduced by (166) and (173). The proof is completed.

From Theorem 47, Theorem 51, and Lemma 54 we obtain the following theorem.

Theorem 55. Assume that $u(x) \in \mathscr{D}(\Lambda)$ satisfies $(L+h)[u] \geq$ 0 , and let $g_{1}, g_{2}, w^{\Delta \nabla}$, and $h(x)$ be bounded on $(a, b)_{\mathbb{T}}$, such that (92) and (166) hold. Assume that there exists a function $w(x) \in \mathscr{D}(\Lambda)$ satisfying $(162)$. If $v(x)=u(x) / w(x)$ attains its nonnegative maximum $M$ in $(a, b)_{\mathbb{T}}$, then $v(x)=u(x) / w(x) \equiv$ $M$. If $v(a)=M$, then $v^{\Delta}(a)<0$; if $v(b)=M$, then $v^{\nabla}(b)>0$.

In Theorem 55, if we take $\mathbb{T}=\mathbb{R}$, we have the following corollary which is the result that appeared in [3].

Corollary 56. Assume that the functions $g, h:[a, b] \rightarrow \mathbb{R}$ are bounded in $(a, b)$, and $u(x) \in \mathscr{D}(\Lambda)$ satisfies $(L+h)[u]:=$ $u^{\prime \prime}+g u^{\prime}+h u \geq 0$ in $(a, b)$. Assume that there exists a function $w(x) \in \mathscr{D}(\Lambda)$ such that

$$
\begin{array}{cc}
w(x)>0 & \text { for each } x \in \Lambda, \\
(L+h)[w] \leq 0 & \text { for each } x \in(a, b) .
\end{array}
$$

If $v(x)=u(x) / w(x)$ attains its nonnegative maximum $M$ in $(a, b)$, then $v(x)=u(x) / w(x) \equiv M$. If $v(a)=M$, then $v^{\prime}(a)<$ 0 ; if $v(b)=M$, then $v^{\prime}(b)>0$.

In Theorem 55, if we take $\mathbb{T}=\mathbb{Z}$, where $\mathbb{Z}$ is the set of all integral numbers, we can obtain the following new maximum principle for second-order mixed $\Delta$ and $\nabla$ difference inequality.

Corollary 57. Assume that the functions $h, w, g_{1}$, and $g_{2}$ : $[a, b]_{\mathbb{Z}} \rightarrow \mathbb{R}$ satisfy $w(k)>0, \forall k \in[a, b]_{\mathbb{Z}}$

$$
\begin{gathered}
1+g_{1}(k)>0, \quad 1-g_{2}(k)>0, \quad \forall k \in(a, b)_{\mathbb{Z}}, \\
\nabla \Delta w(k)+g_{1}(k) \Delta w(k)+g_{2}(k) \nabla w(k) \\
+h(k) w(k) \leq 0, \quad \forall k \in(a, b)_{\mathbb{Z} .}
\end{gathered}
$$

Let $u(x) \in \mathscr{D}(\Lambda)$ satisfy

$$
\begin{gathered}
\nabla \Delta u(k)+g_{1}(k) \Delta u(k)+g_{2}(k) \nabla u(k) \\
+h(k) u(k) \geq 0, \quad \forall k \in(a, b)_{\mathbb{Z}} .
\end{gathered}
$$

If $v(x)=u(x) / w(x)$ attains its nonnegative maximum $M$ in $(a, b)_{\mathbb{Z}}$, then $v(x)=u(x) / w(x) \equiv M$. If $v(a)=M$, then $v(a+$ $1)<v(a)$; if $v(b)=M$, then $v(b-1)>v(b)$.

To show the value of Theorem 55, we need the following definition.

Definition 58. One says that $x_{0}$ is a change sign point of $u$, if there exist $\delta>0$ and $\eta>0$, such that $u(x)$ has different sign on $\left(x_{0}-\delta, x_{0}\right)_{\mathbb{T}}$ and $\left[x_{0}, x_{0}+\eta\right)_{\mathbb{T}}$, that is, either $u(x)>0$ on $\left(x_{0}-\delta, x_{0}\right)_{\mathbb{T}}$ and $u(x) \leq 0$ on $\left[x_{0}, x_{0}+\eta\right)_{\mathbb{T}}$ or $u(x)<0$ on $\left(x_{0}-\delta, x_{0}\right)_{\mathbb{T}}$ and $u(x) \geq 0$ on $\left[x_{0}, x_{0}+\eta\right)_{\mathbb{T}}$.

Remark 59. Theorem 55 shows that a function $u$ which satisfies (151) cannot oscillate too rapidly. In fact, assuming that $u>0$ between two of its change sign points $x=a$, $x=b$, then $u / w$ must have a positive maximum between them. Hence, Theorem 55 will be violated. Thus, we have the following corollary.

Corollary 60. Assuming $u(x) \in \mathscr{D}(\Lambda)$ satisfies $(L+h)[u] \geq 0$, then $u$ can have at most two change sign points (between which $u$ is negative) in any interval $(a, b)_{\mathbb{T}}$ in which Theorem 55 holds.

By applying the same reasoning to both $u$ and $-u$, we can obtain the following corollary.

Corollary 61. If $u(x)$ is a solution of equation $u^{\Delta \nabla}+g_{1} u^{\Delta}+$ $g_{2} u^{\nabla}+h u=0$, then $u$ can have at most one change sign point in any interval $(a, b)_{\mathbb{T}}$ in which Theorem 55 holds.

Theorem 55 depends on the existence of the function $w$, and now, we discuss the existence of the function $w$.

Lemma 62. Assume that $g_{1}(x), g_{2}(x)$, and $h(x)$ satisfy the suppositions of Theorem 55, and there are positive numbers $M, L$, such that the following properties hold. 
(a) $2 M<\frac{1}{(b-a)^{2}}$.

(b) $\min _{x \in[a, b]_{\pi}}\left\{1+g_{1}(x) \int_{\sigma(a)}^{x} \nabla q+g_{2}(x) \int_{\sigma(a)}^{\rho(x)} \nabla q+\right.$ $\left.h(x) \int_{a}^{x}\left(\int_{\sigma(a)}^{y} \nabla q\right) \Delta y\right\}>L / 2$.

(c) $|h(x)| \leq M$.

Then there exists a function $w(x)$ satisfying (162) and $w^{\Delta \nabla}$ is bounded in $(a, b)_{\mathbb{T}}$.

Proof. We can choose

$$
w(x)=L-\beta \int_{a}^{x}\left(\int_{\sigma(a)}^{y} \nabla q\right) \Delta y,
$$

and then

$$
\begin{gathered}
w^{\nabla}(x)=-\beta \int_{\sigma(a)}^{\rho(x)} \nabla q, \quad w^{\Delta}(x)=-\beta \int_{\sigma(a)}^{x} \nabla q \\
w^{\Delta \nabla}(x)=-\beta .
\end{gathered}
$$

Moreover,

$$
\begin{array}{r}
(L+h)[w]=-\beta\left\{1+g_{1} \int_{\sigma(a)}^{x} \nabla q+g_{2} \int_{\sigma(a)}^{\rho(x)} \nabla q\right. \\
\left.+h \int_{a}^{x}\left(\int_{\sigma(a)}^{y} \nabla q\right) \Delta y\right\}+h L .
\end{array}
$$

Since $|h(x)| \leq M$,

$$
\begin{array}{r}
\min _{x \in[a, b]_{\mathbb{T}}}\left\{1+g_{1} \int_{\sigma(a)}^{x} \nabla q+g_{2} \int_{\sigma(a)}^{\rho(x)} \nabla q\right. \\
\left.+h \int_{a}^{x}\left(\int_{\sigma(a)}^{y} \nabla q\right) \Delta y\right\}>\frac{L}{2} .
\end{array}
$$

Then $\forall \beta: 2 M<\beta<\left(L /(b-a)^{2}\right)$; we have

$$
\begin{gathered}
(L+h)[w] \leq 0, \\
\beta \int_{a}^{x}\left(\int_{\sigma(a)}^{y} \Delta q\right) \nabla y<L,
\end{gathered}
$$

and hence

$$
w(x) \geq L-\beta(b-a)^{2}>0 .
$$

Lemma 63. Let $r(x)$ be a solution of equation

$$
r^{\Delta \nabla}+g_{1} r^{\Delta}+g_{2} r^{\nabla}+h r=0
$$

where $g_{1}, g_{2}, h$, and $w$ satisfy the conditions of Theorem 55. If $r$ is not identically zero and

$$
r(a)=0
$$

then $r$ cannot vanish in some right neighbourhood of a.
Proof. If $a$ is right-scattered, then $r(\sigma(a)) \neq 0$. Otherwise, we have that $r^{\Delta}(a)=0$; this shows that

$$
\begin{gathered}
v(a)=0, \\
v^{\Delta}(a)=\frac{r^{\Delta}(a) w(a)-r(a) w^{\Delta}(a)}{w(a) w(\sigma(a))}=0 .
\end{gathered}
$$

Then we can obtain $v(x) \equiv 0$. In fact, according to Theorem 55, $v=r / w$ cannot attain its maximum nor minimum at $a$. If $v$ attains its maximum in $(a, b)_{\mathbb{T}}$, then $v \equiv 0$ since $v(a)=0$. If $v$ attains its maximum at $b$, hence $-v$ attains its maximum in $(a, b)_{\mathbb{T}}$. Next we apply Theorem 47 to $-v$ and obtain that $-v(x)$ is constant; then $v(x) \equiv 0$ since $-v(a)=0$. Thus, in all cases we get that $v(x) \equiv 0$; this implies that $r(x) \equiv 0$ which is contradiction with the assumption.

If $a$ is right-dense, we obtain that $r$ cannot vanish in some right neighbourhood of $a$. In fact, if it is not so, then there exists a sequence $t_{n} \rightarrow a^{+}$, and $r\left(t_{n}\right)=0$; then $r^{\Delta}(a)=$ $\lim _{n \rightarrow \infty}\left(r(a)-r\left(t_{n}\right)\right) /\left(a-t_{n}\right)=0$. Again we obtain that $r(x) \equiv 0$ by a similar proof of above, which is contradiction with the assumption. Thus, $r$ cannot vanish in some right neighbourhood of $a$.

Remark 64. Under the conditions of Lemma 63, if $r$ has any change sign point at the right of $a$, we denote the first one by $a^{*}$ and call it the conjugate change sign point of $a$. Thus, $r$ does not change its sign in the interval $\left(a, a^{*}\right)_{\mathbb{T}}$. Without loss of the generality, we assume that

$$
r(x)>0 \text { for } x \in\left(a, a^{*}\right)_{\mathbb{T}} .
$$

Then function $r / w$ is positive in $\left(a, a^{*}\right)_{\mathbb{T}}$ and $a^{*}$ is also a change sign point of $r / w$. By the definition of change sign point, we have that $r\left(a^{*}\right) / w\left(a^{*}\right) \leq 0$. Hence, $r / w$ has a maximum in $\left(a, a^{*}\right)_{\mathbb{T}}$. Therefore by Theorem $55, w$ cannot satisfy $(L+h)[w] \leq 0$. That is, under these cases, there is no function $w$ satisfying the condition of Theorem 51 .

On the other hand, if $b$ is any point in $\left(a, a^{*}\right)_{\mathbb{T}}$, a function $w$ can be found so that $r / w$ satisfies the maximum principle of Theorem 55. To see this, we observe first that $r(x)$ is bounded from below by a positive number on any subinterval $[c, b]_{\mathbb{T}}$ contained in $\left(a, a^{*}\right)_{\mathbb{T}}$. Consequently, for sufficiently small $\varepsilon>$ 0 , the function $w(x)=r(x)+\varepsilon\left(2-e_{\alpha}(x, a)\right)$ is positive on $[a, b]_{\mathbb{T}}$. If $\alpha$ is selected so that $(L+h)\left[2-e_{\alpha}(x, a)\right] \leq 0$ in $(a, b)_{\mathbb{T}}$, then $w$ is a function for which Theorem 55 holds. Thus, we get the following result.

Theorem 65. If $a^{*}$ is the conjugate change sign point of $a$, letting $g_{1}, g_{2}$, and $h(x)$ be bounded on $(a, b)_{\mathbb{T}}$, such that (92) and (166) hold, then there exists a $w(x)>0$ such that Theorem 55 holds on the interval $[a, b]_{\mathbb{T}}$ if and only if $b<a^{*}$. If $r(x)$ (the solution of (186) which satisfies $r(a)=0$ ) has no change sign point at the right of $a$, one sets $a^{*}=\infty$, and Theorem 55 holds on every interval $[a, b]_{\mathbb{T}}$.

In Theorem 65 , if we take $\mathbb{T}=\mathbb{R}$, we have the following corollary which is the result that appeared in [3].

Corollary 66. Assume that $a^{*}$ is the conjugate change sign point of $a$, and the functions $g, h:[a, b] \rightarrow \mathbb{R}$ are bounded 
in $(a, b)$; then there exists a function $w(x)>0$ such that Corollary 52 holds on the interval $[a, b]$ if and only if $b<a^{*}$. If $r(x)$ (the solution of $r^{\prime \prime}+g r^{\prime}+h r=0$, which satisfies $r(a)=0$ ) has no change sign point at the right of $a$, one sets $a^{*}=\infty$, and Corollary 52 holds on every interval $[a, b]$.

In Theorem 65, if we take $\mathbb{Z}=\mathbb{Z}$, where $\mathbb{Z}$ is the set of all integral numbers, we can obtain the following new maximum principle for second-order mixed $\Delta$ and $\nabla$ difference inequality.

Corollary 67. Assuming that the functions $h, w, g_{1}$, and $g_{2}$ : $[a, b]_{\mathbb{Z}} \rightarrow \mathbb{R}$ satisfy

$$
1+g_{1}(k)>0, \quad 1-g_{2}(k) \geq 0, \quad \forall k \in(a, b)_{\mathbb{Z}},
$$

then there exists a function $w(k)>0, \forall k \in[a, b]_{\mathbb{Z}}$, such that Corollary 53 holds on the interval $[a, b]_{\mathbb{Z}}$ if and only if $b<a^{*}$. If $r(x)$ (the solution of the equation

$$
\begin{aligned}
& \nabla \Delta r(k)+g_{1}(k) \Delta r(k)+g_{2}(k) \nabla r(k) \\
& \quad+h(k) r(k)=0,
\end{aligned}
$$

which satisfies $r(a)=0$ ) has no change sign point at the right of $a$, one sets $a^{*}=\infty$, and Corollary 53 holds on every interval $[a, b]_{\mathbb{Z}}$

\section{Applications to Initial Value Problems}

In this section, as an application of the maximum principles established in section three, firstly, we will prove some uniqueness theorem of the solution for initial value problem:

$$
\begin{gathered}
u^{\Delta \nabla}+g_{1} u^{\Delta}+g_{2} u^{\nabla}+h u=f(x) \quad \text { on }(a, b)_{\mathbb{T}}, \\
u(a)=\gamma_{1}, \quad u^{\Delta}(a)=\gamma_{2},
\end{gathered}
$$

in $\mathscr{D}(\Lambda)$. Secondly, we will discuss the existence of the lower and upper solutions of (192). Thirdly, we will give a general scheme for obtaining upper and lower solutions.

Theorem 68. Assume that $g_{1}, g_{2}$, and $h$ satisfy (91) and (92), and $h \leq 0,(93)$, (130) hold on $(a, b)_{\mathbb{T}}$. If $u_{1}$ and $u_{2}$ are solutions of the initial value problem (192), then $u_{1} \equiv u_{2}$.

Proof. We define a function $v(x) \in \mathscr{D}(\Lambda)$ by

$$
v(x)=u_{1}(x)-u_{2}(x) .
$$

Since both $u_{1}$ and $u_{2}$ satisfy (192), the function $v$ satisfies

$$
\begin{aligned}
& v^{\Delta \nabla}+g_{1} v^{\Delta}+g_{2} v^{\nabla}+h v=0, \\
& v(a)=0, \quad v^{\Delta}(a)=0 .
\end{aligned}
$$

According to Theorem 51, $v$ cannot attain its maximum nor minimum at $a$. If $v$ attains its maximum at anterior point of $\Lambda, v \equiv 0$ since $v(a)=0$. If $v$ attains its maximum at $b$, hence $-v$ attains its maximum at an interior point of $\Lambda$. Next we apply Theorem 47 to $-v$ and obtain that $-v(x)$ is constant; then $v(x) \equiv 0$ since $-v(a)=0$. The proof is completed.

It follows from Theorem 65; we get Theorem 69.
Theorem 69. Let $g_{1}, g_{2}$, and $h(x)$ be bounded on $(a, b)_{\mathbb{T}}$, such that (92) and (166) hold. Assuming that $u_{1}$ and $u_{2}$ are solutions of the initial value problem (192), if $b<a^{*}$, where $a^{*}$ is the conjugate change sign point of $a$, then $u_{1} \equiv u_{2}$.

More generally, we can prove the following theorem which shows that the conclusion of Theorem 69 holds on any interval $[a, b]_{\mathbb{T}}$.

Theorem 70. Let $g_{1}, g_{2}$, and $h(x)$ be bounded on $(a, b)_{\mathbb{T}}$, such that (92) and (166) hold. Assuming that $u_{1}$ and $u_{2}$ are solutions of the initial value problem (192), then $u_{1} \equiv u_{2}$.

Proof. We define a function $u(x) \in \mathscr{D}(\Lambda)$ by

$$
u(x)=u_{1}(x)-u_{2}(x) .
$$

Since both $u_{1}$ and $u_{2}$ satisfy (192), the function $u$ satisfies (194). We give our proof by two steps.

(1) If $a$ is right-scattered, it follows from (194) that $u(\sigma(a))=0, u^{\nabla}(\sigma(a))=u^{\Delta}(a)=0$, and then we have that $u^{\Delta \nabla}(\sigma(a))+g_{1}(\sigma(a)) u^{\Delta}(\sigma(a))=0$. On the other hand,

$$
u^{\Delta \nabla}(\sigma(a))=\frac{u^{\Delta}(a)-u^{\Delta}(\sigma(a))}{a-\sigma(a)},
$$

this implies that

$$
\begin{aligned}
& \frac{u^{\Delta}(a)-u^{\Delta}(\sigma(a))}{a-\sigma(a)}+g_{1}(\sigma(a)) u^{\Delta}(\sigma(a)) \\
& =\frac{u^{\Delta}(\sigma(a))\left[1+v(\sigma(a)) g_{1}(\sigma(a))\right]}{v(\sigma(a))}=0 .
\end{aligned}
$$

Note that (166); we know that $1+\nu(\sigma(a)) g_{1}(\sigma(a))>0$. This shows that $u^{\Delta}(\sigma(a))=0$. If $a$ is right-dense, by using Lemma 62, there is a $\varepsilon>0$ enough small and a function $w(x)>0$ on $[a, a+\varepsilon]_{\mathbb{T}}$. Let $v=u / w$; then

$$
\begin{gathered}
v(a)=0, \\
v^{\Delta}(a)=\frac{u^{\Delta}(a) w(a)-u(a) w^{\Delta}(a)}{w(a) w(\sigma(a))}=0, \\
v^{\Delta \nabla}+\frac{F^{\Delta \nabla}+g_{1} w^{\sigma}}{w^{\sigma \rho}} v^{\Delta}+\frac{w^{\Delta \rho}+g_{2} w^{\rho}}{w^{\sigma \rho}} v^{\nabla} \\
+\frac{1}{w^{\sigma \rho}}(L+h)[w] v=0 .
\end{gathered}
$$

According to Theorem 55, $v=r / w$ cannot attain its maximum nor minimum at $a$. If $v$ attains its maximum in $(a, a+\varepsilon)_{\mathbb{T}}$, then $v \equiv 0$ since $v(a)=0$. If $v$ attains its maximum at $a+\varepsilon$, hence $-v$ attains its maximum in $(a, a+\varepsilon)_{\mathbb{T}}$. Next we apply Theorem 55 to $-v$ and obtain that $-v(x)$ is constant; then $v(x) \equiv 0$ since $-v(a)=0$. Thus, in all cases we get that $v(x) \equiv 0$; this implies that $u(x) \equiv 0$ on $[a, a+\varepsilon]_{\mathbb{T}}$. Thus, we can get that $u^{\Delta}(x) \equiv 0$ on $[a, a+\varepsilon)_{\mathbb{T}}$. If $a+\varepsilon$ is left-dense, by using the continuous $u^{\Delta}(x)$, we have that $u^{\Delta}(a+\varepsilon)=0$. If $a+\varepsilon$ is left-scattered, then $u^{\Delta}(\rho(a+\varepsilon))=0$, and $\rho(a+\varepsilon)$ is rightscattered; then similar to the above proof of $u^{\Delta}(\sigma(a))=0$, we 
have that $u^{\Delta}(a+\varepsilon)=u^{\Delta}(\sigma(\rho(a+\varepsilon)))=0$. Synthesizing the above proof, we have proven that there exists $\varepsilon>0$ (if $a$ is right-scattered, $\varepsilon=\mu(a))$, such that $u(x) \equiv 0$ and $u^{\Delta}(x) \equiv 0$ on $[a, a+\varepsilon]_{\mathbb{T}}$.

(2) Let

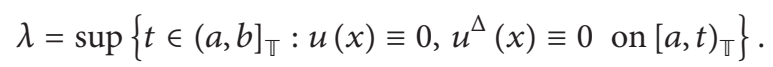

If $\lambda=b$, then the conclusion of Theorem 70 will be proved. If $\lambda<b$, then $u(x) \equiv 0$ and $u^{\Delta}(x) \equiv 0$ on $[a, \lambda)_{\mathbb{T}}$. If $\lambda$ is leftdense, by using the continuous $u^{\Delta}(x)$, we have that $u(\lambda)=$ 0 and $u^{\Delta}(\lambda)=0$. If $\lambda$ is left-scattered, then $u^{\Delta}(\rho(\lambda))=0$, and $\rho(\lambda)$ is right-scattered; then similar to the above proof of $u^{\Delta}(\sigma(a))=0$, we can prove that $u^{\Delta}(\lambda)=u^{\Delta}(\sigma(\rho(\lambda)))=0$. By using the conclusion of step (1), we have that there exists $\varepsilon>0$ such that $u(x) \equiv 0$ and $u^{\Delta}(x) \equiv 0$ on $[\lambda, \lambda+\varepsilon]_{T}$. Then we get that $u(x) \equiv 0$ and $u^{\Delta}(x) \equiv 0$ on $[a, \lambda+\varepsilon]_{\mathbb{T}}$. This is a contradiction with the definition of $\lambda$. Thus, $\lambda=b$; this shows that $u_{1} \equiv u_{2}$ on $[a, b]_{\mathbb{T}}$. The proof is completed.

Remark 71. Theorems 68 and 69 show that $(L+h)[u]=u^{\Delta \nabla}+$ $g_{1} u^{\Delta}+g_{2} u^{\nabla}+h u=f(x)$, at most, has one solution satisfying $u(a)=\gamma_{1}, u^{\Delta}(a)=\gamma_{2}$. On the other hand, in many cases, it is difficult to find a solution of the initial value problem directly, and therefore, it becomes important to find a lower and upper solution.

Assume that $g_{1}, g_{2}$, and $h$ are bounded on $(a, b)_{\mathbb{T}}, h(x) \leq 0$ on $(a, b)_{\pi}$ and satisfy (91), (92), (93), and (130) for each $x \in$ $(a, b)_{\mathbb{T}}$. If we can find a function $z_{1}(x) \in \mathscr{D}(\Lambda)$ satisfying

$$
\begin{gathered}
(L+h)\left[z_{1}\right] \geq f(x), \\
z_{1}(a) \geq \gamma_{1}, \quad z_{1}^{\Delta}(a) \geq \gamma_{2},
\end{gathered}
$$

we define a function $v_{1}(x) \in \mathscr{D}(\Lambda)$ by

$$
v_{1}(x) \equiv z_{1}(x)-u(x)
$$

where $u(x)$ is the solution of (192). Thus,

$$
\begin{gathered}
(L+h)\left[v_{1}\right] \geq 0, \\
v_{1}(a) \geq 0, \quad v_{1}^{\Delta}(a) \geq 0 .
\end{gathered}
$$

Since $v_{1}(a) \geq 0, v_{1}(x)$ has a nonnegative maximum at any interval $\left[a, x_{0}\right]_{\mathbb{T}}$, and using Theorem 47 , we know that the maximum point must be $a$ or $x_{0}$. However, $v_{1}^{\Delta}(a) \geq 0$, and from Theorem 51 maximum point cannot be $a$ unless $v_{1}(x) \equiv$ constant. Thus, we obtain

$$
\max _{x \in\left[a, x_{0}\right]_{\mathbb{T}}} v_{1}(x)=v_{1}\left(x_{0}\right) \geq v_{1}(a), \quad x_{0} \in(a, b)_{\mathbb{T}} .
$$

Since $x_{0} \in(a, b)_{\mathbb{T}}$ is arbitrary, we can deduce that

$$
v_{1}^{\Delta}(x) \geq 0, \quad x \in(a, b)_{\mathbb{T}} .
$$

Using $x$ to take the place of $x_{0}$, inequality (204) implies

$$
u(x) \leq \gamma_{1}+z_{1}(x)-z_{1}(a), \quad x \geq a,
$$

and inequality (205) implies

$$
u^{\Delta}(x) \leq z_{1}^{\Delta}(x), \quad x \in[a, b)_{\mathbb{V}} .
$$

Since

$$
z_{1}(a)-\gamma_{1} \geq 0
$$

inequality (206) implies

$$
u(x) \leq z_{1}(x) .
$$

Similarly, assume that we can find a function $z_{2}(x) \in \mathscr{D}(\Lambda)$ satisfying

$$
\begin{gathered}
(L+h)\left[z_{2}\right] \leq f(x), \\
z_{2}(a) \leq \gamma_{1}, \quad z_{2}^{\Delta}(a) \leq \gamma_{2} .
\end{gathered}
$$

The same as the above statement, define

$$
v_{2}(x) \equiv u(x)-z_{2}(x),
$$

and we obtain

$$
u(x) \geq z_{2}(x), \quad u^{\Delta}(x) \geq z_{2}^{\Delta}(x) .
$$

Therefore, we have established the following theorem, which gives a sufficient condition for the lower and upper solutions.

Theorem 72. Assume that $g_{1}, g_{2}$, and $h$ are bounded on $(a, b)_{\mathbb{T}}, h(x) \leq 0$ on $(a, b)_{\mathbb{T}}$ and satisfy (91), (92), (93), and (130) for each $x \in(a, b)_{\mathbb{T}}$. Let $u(x) \in \mathscr{D}(\Lambda)$ be a solution of (192). Let $z_{1}(x)$ and $z_{2}(x)$ satisfy (200), (201) and (210), (211). Then $z_{2}(x) \leq u(x) \leq z_{1}(x), z_{2}^{\Delta}(x) \leq u^{\Delta}(x) \leq z_{1}^{\Delta}(x)$.

In the following, we will discuss the existence of the lower and upper solutions.

Theorem 73. Assume that $g_{1}, g_{2}$, and $h$ are bounded on $(a, b)_{\mathbb{T}}$, are bounded on $(a, b)_{\mathbb{T}}$, and satisfy (166), (92), (93), and (130).

(1) If $f(x)$ is continuous on $\Lambda$, then there exist functions $z_{1}(x)$ and $z_{2}(x)$ which satisfy (200), (201) and (210), (211), respectively.

(2) Moreover, if $h(x) \leq 0$ on $(a, b)_{\mathbb{T}}$, then $z_{2}(x) \leq u(x) \leq$ $z_{1}(x), z_{2}^{\Delta}(x) \leq u^{\Delta}(x) \leq z_{1}^{\Delta}(x)$.

Proof. It follows from (166) that

$$
1+v g_{1} \geq t_{1}>0, \quad \text { on }(a, b)_{\mathbb{T}},
$$

so we can select $\alpha>0$ large enough, such that $k>0$, where $k$ is defined by

$$
\begin{aligned}
k= & \min _{x \in[a, b]_{\mathbb{T}}}\left[\left(1+\nu g_{1}\right) \alpha^{2}+\alpha\left(g_{1}+g_{2}\right)+h(1+\alpha \nu(x))\right] \\
& \times \frac{e_{\alpha}(x, a)}{1+\alpha \nu} .
\end{aligned}
$$


Let

$$
A=\max \left\{\gamma_{1}, \frac{\gamma_{2}}{\alpha}, \frac{1}{k} \cdot \max _{x \in[a, b]_{\top}}\{f(x)\}, 0\right\}
$$

we show that, under the stated assumptions, the function

$$
z_{1}(x):=A e_{\alpha}(x, a)
$$

satisfies (200) and (201). To see that (200) is satisfied, we note that

$$
\begin{aligned}
(L+ & h)\left[z_{1}\right] \\
= & A\left[\left(1+v g_{1}\right) \alpha^{2}+\alpha\left(g_{1}+g_{2}\right)+h(1+\alpha v)\right] \\
& \times \frac{e_{\alpha}(x, a)}{1+\alpha \nu} \\
\geq & A k \\
\geq & \frac{1}{k} \cdot \max _{x \in[a, b]_{\top}}\{f(x)\} \cdot k \\
\geq & f(x) .
\end{aligned}
$$

To see that (201) is satisfied, we note that

$$
z_{1}(a)=A \geq \gamma_{1}, \quad z_{1}^{\Delta}(a)=A \alpha \geq \gamma_{2} .
$$

Similarly, we can choose

$$
z_{2}(x):=B e_{\alpha}(x, a),
$$

where

$$
B=\min \left\{\gamma_{1}, \frac{\gamma_{2}}{\alpha}, \frac{1}{k} \cdot\left\{\min _{x \in[a, b]_{T}} f(x)\right\}, 0\right\} .
$$

To see that (210) is satisfied, we note that

$$
\begin{aligned}
(L+ & h)\left[z_{2}\right] \\
= & B\left[\left(1+\nu g_{1}\right) \alpha^{2}+\alpha\left(g_{1}+g_{2}\right)+h(1+\alpha \nu(x))\right] \\
& \times \frac{e_{\alpha}(x, a)}{1+\alpha \nu} \\
\leq & B k \\
\leq & \frac{1}{k} \cdot \min _{x \in[a, b]_{\top}}\{f(x)\} \cdot k \\
\leq & f(x) .
\end{aligned}
$$

To see that (211) is satisfied note that

$$
z_{2}(a)=B \leq \gamma_{1}, \quad z_{2}^{\Delta}(a)=B \alpha \leq \gamma_{2} .
$$

Thus, conclusion (1) holds. Conclusion (2) can be deduced from Theorem 72 .

The proof is completed.
As we all know, the accuracy of the approximation will depend on how well we can choose the functions $z_{1}(x)$ and $z_{2}(x)$. So we next search for the following general scheme for obtaining upper and lower bounds. Suppose we divide the interval $[a, b]_{\mathbb{T}}$ into $N$ subintervals

$$
a=x_{0}<x_{1}<\cdots<x_{N-1}<x_{N}=b .
$$

On each subinterval, we will select $z_{1}(x)$ as the following form:

$$
c \int_{t}^{x}\left(\int_{t}^{y} \nabla q\right) \Delta y+d \int_{t}^{x} \nabla q+e
$$

and choose the coefficients $c$, $d$, $e$ so that $z_{1}(a)=\gamma_{1}, z_{1}^{\Delta}(a)=$ $\gamma_{2}$, and $z_{1} \in \mathscr{D}(\Lambda)$. Also, $z_{1}$ will be selected so that inequality $(200)$ holds in each subinterval $\left(x_{i-1}, x_{i}\right)_{\mathbb{T}}$. We set

$$
\begin{array}{r}
z_{1}(x)=c_{i} \int_{x_{\mathrm{i}}}^{x}\left(\int_{x_{i}}^{y} \nabla q\right) \Delta y+d_{i} \int_{x_{i}}^{x} \nabla q+e_{i} \\
\text { for } x \in\left[x_{i}, x_{i+1}\right)_{\mathbb{T}}, i=0,1, \ldots, N-1 .
\end{array}
$$

The constants $c_{i}, d_{i}, e_{i}, i=0,1,2, \ldots, N-1$, and the number $N$ of subintervals will be chosen so that all the required conditions are satisfied. We proceed in a step by step manner starting with the interval $\left(x_{0}, x_{1}\right)_{\mathbb{T}}$. The initial conditions

$$
z_{1}(a)=\gamma_{1}, \quad z_{1}^{\Delta}(a)=\gamma_{2},
$$

require that $e_{0}=\gamma_{1}$ and $d_{0}=\gamma_{2}$. Next, we divide our proof into three parts.

(i) If $x_{0}$ is right-scattered and $\sigma\left(x_{0}\right)$ is also right-scattered, we let $x_{1}=\sigma\left(\sigma\left(x_{0}\right)\right)$, and then we only have one point $\sigma\left(x_{0}\right)$ in $\left(x_{0}, x_{1}\right)_{\mathbb{T}}$, and hence, in this point, the inequality

$$
(L+h)\left[z_{1}\right] \geq f(x)
$$

becomes

$$
\begin{aligned}
c_{0}[1 & \left.+g_{1}^{\sigma}\left(x_{0}\right) v\left(\sigma\left(x_{0}\right)\right)\right]+\gamma_{2}\left(g_{1}^{\sigma}\left(x_{0}\right)+g_{2}^{\sigma}\left(x_{0}\right)\right) \\
& +h\left(\sigma\left(x_{0}\right)\right)\left(\gamma_{2} \int_{x_{0}}^{\sigma\left(x_{0}\right)} \nabla q+\gamma_{1}\right) \\
\geq & f\left(\sigma\left(x_{0}\right)\right) .
\end{aligned}
$$

If $1+g_{1} v \geq t_{1}>0$ and $g_{1}, g_{2}, h$, and $f$ are bounded, then $c_{0}$ can be properly selected so that (229) is an equality. Thus, in this case, $z_{1}(x)$ is a solution of $(192)$ in $\left(x_{0}, x_{1}\right)_{\mathbb{T}}$.

(ii) If $x_{0}$ is right-scattered and $\sigma\left(x_{0}\right)$ is right-dense, we let $x_{1}>\sigma\left(x_{0}\right)$, and then the inequality

$$
(L+h)\left[z_{1}\right] \geq f(x), \quad x \in\left(x_{0}, x_{1}\right)_{\mathbb{T}},
$$

becomes

$$
\begin{aligned}
& c_{0}\left[1+g_{1}(x)\left(\int_{x_{0}}^{x} \nabla q\right)+g_{2}(x)\left(\int_{x_{0}}^{\rho(x)} \nabla q\right)\right. \\
& \left.+h(x) \int_{x_{0}}^{x}\left(\int_{x_{0}}^{y} \nabla q\right) \Delta y\right]+\gamma_{2}\left(g_{1}(x)+g_{2}(x)\right) \\
& +h(x)\left(\gamma_{2} \int_{x_{0}}^{x} \nabla q+\gamma_{1}\right) \\
& \geq f(x), \quad x \in\left(x_{0}, x_{1}\right)_{\mathbb{T}} .
\end{aligned}
$$


If $x=\sigma\left(x_{0}\right)$, we have that

$$
\begin{aligned}
c_{0}\left[1+g_{1}\left(\sigma\left(x_{0}\right)\right)\left(\int_{x_{0}}^{\sigma\left(x_{0}\right)} \nabla q\right)\right. \\
+g_{2}\left(\sigma\left(x_{0}\right)\right)\left(\int_{x_{0}}^{\rho\left(\sigma\left(x_{0}\right)\right)} \nabla q\right) \\
\left.+h\left(\sigma\left(x_{0}\right)\right) \int_{x_{0}}^{\sigma\left(x_{0}\right)}\left(\int_{x_{0}}^{y} \nabla q\right) \Delta y\right] \\
+\gamma_{2}\left(g_{1}\left(\sigma\left(x_{0}\right)\right)+g_{2}\left(\sigma\left(x_{0}\right)\right)\right) \\
+h\left(\sigma\left(x_{0}\right)\right)\left(\gamma_{2} \int_{x_{0}}^{\sigma\left(x_{0}\right)} \nabla q+\gamma_{1}\right) \\
=c_{0}\left[1+g_{1}\left(\sigma\left(x_{0}\right)\right) \nu\left(\sigma\left(x_{0}\right)\right)\right] \\
+\gamma_{2}\left(g_{1}\left(\sigma\left(x_{0}\right)\right)+g_{2}\left(\sigma\left(x_{0}\right)\right)\right) \\
+h\left(\sigma\left(x_{0}\right)\right)\left(\gamma_{2} v\left(\sigma\left(x_{0}\right)\right)+\gamma_{1}\right) \\
\geq
\end{aligned}
$$

Thus, if $g_{1}, g_{2}, f$, and $h$ are bounded, then $x_{1}$ can be selected so close to $\sigma\left(x_{0}\right)$, and $c_{0}$ can be taken so large that (231) holds for $x \in\left(x_{0}, x_{1}\right)_{\mathbb{T}}$. Moreover, when $x_{1}$ is sufficiently close to $\sigma\left(x_{0}\right)$, we can properly select $c_{0}$, such that (231) is close to an equality; then $z_{1}(x)$ is also close to the solution of (192) in $\left(x_{0}, x_{1}\right)_{\mathbb{T}}$.

(iii) If $x_{0}$ is right-dense, the inequality

$$
(L+h)\left[z_{1}\right] \geq f(x), \quad x \in\left(x_{0}, x_{1}\right)_{\mathbb{T}},
$$

becomes

$$
\begin{gathered}
c_{0}\left[1+g_{1}(x)\left(\int_{x_{0}}^{x} \nabla q\right)+g_{2}(x)\left(\int_{x_{0}}^{\rho(x)} \nabla q\right)\right. \\
\left.+h(x) \int_{x_{0}}^{x}\left(\int_{x_{0}}^{y} \nabla q\right) \Delta y\right] \\
\quad+\gamma_{2}\left(g_{1}+g_{2}\right)+h\left(\gamma_{2} \int_{x_{0}}^{x} \nabla q+\gamma_{1}\right) \\
\geq f(x), \quad x \in\left(x_{0}, x_{1}\right)_{\mathbb{T}} .
\end{gathered}
$$

If $g_{1}, g_{2}$, and $h$ are bounded, then $x_{1}$ can be selected so close to $x_{0}$ that

$$
\begin{gathered}
{\left[1+g_{1}(x)\left(\int_{x_{0}}^{x} \nabla q\right)+g_{2}(x)\left(\int_{x_{0}}^{\rho(x)} \nabla q\right)\right.} \\
\left.+h(x) \int_{x_{0}}^{x}\left(\int_{x_{0}}^{y} \nabla q\right) \Delta y\right] \\
\geq l>0, \quad \text { for } x \in\left(x_{0}, x_{1}\right)_{\mathbb{T}},
\end{gathered}
$$

where $l>0$ is a positive constant. If, in addition, $f$ is bounded, then $c_{0}$ can be taken so large that (234) holds for all $x$ in $\left(x_{0}, x_{1}\right)_{\mathbb{T}}$. Moreover, when $x_{1}$ is sufficiently close to $x_{0}$, we can properly select $c_{0}$, such that (234) is close to an equality; then $z_{1}(x)$ is also close to the solution of (192) in $\left(x_{0}, x_{1}\right)_{\mathbb{T}}$.

Following all of the above proof, we have proved that there exists an $x_{1}>x_{0}$ and a large enough $c_{0}$, such that (200) holds for all $x$ in $\left(x_{0}, x_{1}\right)_{\mathbb{T}}$ for

$$
\begin{aligned}
z_{1}(x)= & c_{0} \int_{x_{0}}^{x}\left(\int_{x_{0}}^{y} \nabla q\right) \Delta y \\
& +\gamma_{2} \int_{x_{0}}^{x} \nabla q+\gamma_{1} \quad \text { for } x \in\left(x_{0}, x_{1}\right)_{\mathbb{T}} .
\end{aligned}
$$

We now turn to the interval $\left(x_{1}, x_{2}\right)_{\mathbb{T}}$, with $z_{1}(x)$ being defined by

$$
\begin{aligned}
z_{1}(x)= & c_{1} \int_{x_{1}}^{x}\left(\int_{x_{1}}^{y} \nabla q\right) \Delta y \\
& +d_{1} \int_{x_{1}}^{x} \nabla q+e_{1} \quad \text { for } x \in\left(x_{1}, x_{2}\right)_{\mathbb{T}} .
\end{aligned}
$$

To insure the continuity of $z_{1}, z_{1}^{\Delta}$, and $z_{1}^{\nabla}$ at $x_{1}$, we choose

$$
\begin{aligned}
& e_{1}=c_{0} \int_{x_{0}}^{x_{1}}\left(\int_{x_{0}}^{y} \nabla q\right) \Delta y+\gamma_{2} \int_{x_{0}}^{x_{1}} \nabla q+\gamma_{1}, \\
& d_{1}=c_{0} \int_{x_{0}}^{x_{1}} \nabla q+\gamma_{2} .
\end{aligned}
$$

In fact, by computing we get that

$$
\begin{aligned}
& \lim _{x \rightarrow x_{1}} z_{1}(x)=e_{1}=z_{1}\left(x_{1}\right), \\
& \lim _{x \rightarrow x_{1}} z_{1}^{\Delta}(x)=d_{1}=c_{0} \int_{x_{0}}^{x_{1}} \nabla q+\gamma_{2}=z_{1}^{\Delta}\left(x_{1}\right), \\
& \lim _{x \rightarrow x_{1}^{+}} z_{1}^{\nabla}(x)=d_{1}=c_{0} \int_{x_{0}}^{x_{1}} \nabla q+\gamma_{2}, \\
& \lim _{x \rightarrow x_{1}^{-}} z_{1}^{\nabla}(x)=c_{0} \int_{x_{0}}^{\rho\left(x_{1}\right)} \nabla q+\gamma_{2}=z_{1}^{\nabla}\left(x_{1}\right) .
\end{aligned}
$$

Thus, $z_{1}, z_{1}^{\Delta}$ are continuous at $x_{1}$, and $z_{1}^{\nabla}$ is left-dense continuous at $x_{1}$. In the interval $\left(x_{1}, x_{2}\right)_{\mathbb{T}}$, we apply the same reasoning of $\left(x_{0}, x_{1}\right)_{\mathbb{T}}$ to $\left(x_{1}, x_{2}\right)_{\mathbb{T}}$ and get that there exists an $x_{2}>x_{1}$ and a large enough $c_{1}$, such that (200) holds for all $x$ in $\left(x_{1}, x_{2}\right)_{\mathbb{T}}$.

Proceeding in this fashion, we determine each $d_{i}, e_{i}$ so that $z_{1}$ and $z_{1}^{\Delta}$ are continuous everywhere; $z_{1}^{\nabla}$ is leftdense continuous everywhere, and if $x_{i}$ is a left-dense point, we always take interval $\left(x_{i}, x_{i+1}\right)_{\mathbb{T}}$ so small, such that the coefficient of $c_{i}$ satisfies:

$$
\begin{gathered}
{\left[1+g_{1}(x)\left(\int_{x_{i}}^{x} \nabla q\right)+g_{2}(x)\left(\int_{x_{i}}^{\rho(x)} \nabla q\right)\right.} \\
\left.+h(x) \int_{x_{i}}^{x}\left(\int_{x_{i}}^{y} \nabla q\right) \Delta y\right] \\
\geq l_{i}>0, \quad \text { for } x \in\left(x_{0}, x_{1}\right)_{\mathbb{T}},
\end{gathered}
$$


where $l_{i}>0$ is a positive constant. Also, we take the constant $c_{i}$ to be large enough, so that $(L+h)\left[z_{1}\right] \geq f(x)$ holds on $\left(x_{i}, x_{i+1}\right)_{\mathbb{T}}$. In fact, the quantities $d_{i}, e_{i}$ are determined by the recursion formulas

$$
\begin{aligned}
& e_{i}=c_{i-1} \int_{x_{i-1}}^{x_{i}}\left(\int_{x_{i}-1}^{y} \nabla q\right) \Delta y+d_{i-1} \int_{x_{i-1}}^{x_{i}} \nabla q+e_{i-1}, \\
& d_{i}=c_{i-1} \int_{x_{i-1}}^{x} \nabla q+d_{i-1} .
\end{aligned}
$$

In an actual computation to determine the $c_{i}$, it is convenient to replace $f$ by its maximum in the $i$ th subinterval and to replace $g_{1}, g_{2}$, and $h$ by either their maximum or minimum, whichever may be appropriate for making $(L+h)\left[z_{1}\right] \geq f(x)$ throughout.

In a similar manner we may construct lower bounds. The constants $d_{i}, e_{i}$ are selected in exactly the same way, and the quantities $-c_{i}$ are taken so large that $(L+h)\left[z_{2}\right] \leq f(x)$ holds everywhere.

If $f, g_{1}, g_{2}$, and $h$ are continuous, by the above process, it can be shown that, as the maximum length of the subintervals, the upper and lower bounds both tend to the solution $u$. The above discussion leads to the following theorem.

Theorem 74. Assume that $g_{1}, g_{2}$, and $h$ are bounded and continuous on $(a, b)_{\mathbb{T}}$ and satisfy (166), (92), (93), and (130). If $f(x)$ is continuous on $\Lambda$ and $h(x) \leq 0$ on $(a, b)_{\mathbb{T}}$, then there exist the upper solution sequence $\left\{z_{1}^{(n)}(x)\right\}$ and lower solution $\left\{z_{2}^{(n)}(x)\right\}$; they both tend to the solution $u$ of (192).

Thus far in this section, we have assumed that $h(x) \leq 0$. We now take up the problem of approximating the solution of the equation

$$
(L+h)[u]=u^{\Delta \nabla}+g_{1} u^{\Delta}+g_{2} u^{\nabla}+h u=f(x)
$$

with initial conditions

$$
u(a)=\gamma_{1}, \quad u^{\Delta}(a)=\gamma_{2}
$$

when the function $h(x)$ may be positive. Under these circumstances we employ the generalized maximum principle (Theorem 51). To do so, we suppose that there is a function $w$ which is positive on $[a, b]_{\mathbb{T}}$ and which has property that

$$
(L+h)[w] \leq 0 \quad \text { for each } x \in[a, b]_{\mathbb{T}} .
$$

For example, we can take the function

$$
w(x)=L-\beta \int_{a}^{x}\left(\int_{\sigma(a)}^{y} \nabla q\right) \Delta y,
$$

defined in Lemma 62.

We saw in Section 3 that $v=u / w$ satisfies an equation of the form

$$
(\bar{L}+H)[v]=v^{\Delta \nabla}+G_{1} v^{\Delta}+G_{2} v^{\nabla}+H v=\frac{f}{w^{\sigma \rho}}
$$

with $G_{1}=\left(F^{\Delta \nabla}+g_{1} w^{\sigma}\right) / w^{\sigma \rho}, G_{2}=\left(w^{\Delta \rho}+g_{2} w^{\rho}\right) / w^{\sigma \rho}$, $H=\left(1 / w^{\sigma \rho}\right)(L+h)[w] \leq 0$. Now, we define the comparison functions $z_{1}(x)$ and $z_{2}(x)$, so that $z_{1} / w$ and $z_{2} / w$ provide the bounds for $u / w$. First, we take $z_{1}(x)$ and $z_{2}(x)$, such that the inequalities

$$
\begin{aligned}
& (L+h)\left[z_{1}\right] \geq f(x), \quad z_{1}(a) \geq \gamma_{1} \\
& z_{1}^{\Delta}(a) w(a)-z_{1}(a) w^{\Delta}(a) \\
& \quad \geq \gamma_{2} w(a)-\gamma_{1} w^{\Delta}(a), \\
& (L+h)\left[z_{2}\right] \leq f(x), \quad z_{2}(a) \leq \gamma_{1} \\
& z_{2}^{\Delta}(a) w(a)-z_{2}(a) w^{\Delta}(a) \\
& \quad \leq \gamma_{2} w(a)-\gamma_{1} w^{\Delta}(a)
\end{aligned}
$$

hold. Then, at $x=a$,

$$
\frac{z_{2}}{w} \leq \frac{u}{w} \leq \frac{z_{1}}{w}, \quad\left(\frac{z_{2}}{w}\right)^{\Delta} \leq\left(\frac{u}{w}\right)^{\Delta} \leq\left(\frac{z_{1}}{w}\right)^{\Delta} .
$$

Moreover, it is easily seen by computing that

$$
(\bar{L}+H)\left[\frac{z_{2}}{w}\right] \leq(\bar{L}+H)\left[\frac{u}{w}\right] \leq(\bar{L}+H)\left[\frac{z_{1}}{w}\right]
$$

Hence, if the conditions of Lemma 54 hold, by using Lemma 54 and Theorem 72, we know that, for $x \in[a, b]_{\mathbb{T}}$,

$$
\begin{gathered}
\frac{z_{2}}{w} \leq \frac{u}{w} \leq \frac{z_{1}}{w}, \\
\left(\frac{z_{2}}{w}\right)^{\Delta} \leq\left(\frac{u}{w}\right)^{\Delta} \leq\left(\frac{z_{1}}{w}\right)^{\Delta} .
\end{gathered}
$$

The first of these sets of inequalities gives the bounds

$$
z_{2}(x) \leq u(x) \leq z_{1}(x)
$$

The second set yields

$$
\begin{aligned}
& z_{2}^{\Delta}(x) w(x)-z_{2}(x) w^{\Delta}(x) \\
& \quad \leq u^{\Delta}(x) w(x)-u(x) w^{\Delta}(x) \\
& \quad \leq z_{1}^{\Delta}(x) w(x)-z_{1}(x) w^{\Delta}(x) .
\end{aligned}
$$

Since $w$ is positive on $[a, b]_{\mathbb{T}}$, we find

$$
\begin{aligned}
& z_{2}^{\Delta}(x)+\frac{w^{\Delta}(x)}{w(x)}\left[u(x)-z_{2}(x)\right] \\
& \leq u^{\Delta}(x) \leq z_{1}^{\Delta}(x)-\frac{w^{\Delta}(x)}{w(x)} \\
& \quad \times\left[z_{1}(x)-u(x)\right]
\end{aligned}
$$

If $w^{\Delta}(x) \leq 0$, we may substitute the upper bound of $u(x)$ as given in (251) into the left side of (253) and we may substitute the lower bound of $u(x)$ into the right side of (253). 
If $w^{\Delta}(x) \geq 0$, we use the lower bound of $u(x)$ on the left and the upper bound of $u(x)$ on the right. We thus find that

$$
\begin{aligned}
& z_{2}^{\Delta}(x)-\frac{-w^{\Delta}(x)}{w(x)}\left[z_{1}(x)-z_{2}(x)\right] \\
& \leq u^{\Delta}(x) \\
& \leq z_{1}^{\Delta}(x)+\frac{-w^{\Delta}(x)}{w(x)}\left[z_{1}(x)-z_{2}(x)\right], \\
& z_{2}^{\Delta}(x) \leq u^{\Delta}(x) \leq z_{1}^{\Delta}(x), \\
& \text { if } w^{\Delta}(x) \geq 0 .
\end{aligned}
$$

Inequalities (251) and (254) give the bounds for $u(x)$ and $u^{\Delta}(x)$ which are more precise when $z_{1}(x)-z_{2}(x)$ and $z_{1}^{\Delta}(x)-$ $z_{2}^{\Delta}(x)$ are smaller.

It is always possible to find a positive function $w$ which satisfies $(L+h)[w] \leq 0$ on a sufficiently small interval, but in general, there is no such function if the interval is too large. Once more we resort to breaking up the interval and piecing together functions defined on subintervals. Let $w>0$ and $(L+h)[w] \leq 0$ on an interval $\left[a, x^{*}\right]_{\mathbb{T}}$, and let $w^{*}$ be another positive function which satisfies $(L+h)\left[w^{*}\right] \leq 0$ on an interval $\left[x^{*}, b\right]_{\mathbb{T}}$. We wish to find bounds for the solution $u$ of the initial value problem (192), on the whole interval $[a, b]_{\mathbb{T}}$.

Let $z_{1}(x)$ and $z_{2}(x)$ satisfy the conditions

$$
(L+h)\left[z_{2}\right] \leq f(x) \leq(L+h)\left[z_{1}\right]
$$

on the interval $\left[a, x^{*}\right]_{\mathbb{T}}$, and

$$
\begin{aligned}
& z_{2}(a) \leq \gamma_{1} \leq z_{1}(a) \\
& z_{2}^{\Delta}(a) w(a)-z_{2}(a) w^{\Delta}(a) \\
& \quad \leq \gamma_{2} w(a)-\gamma_{1} w^{\Delta}(a) \\
& \quad \leq z_{1}^{\Delta}(a) w(a)-z_{1}(a) w^{\Delta}(a) .
\end{aligned}
$$

Then

$$
\begin{gathered}
z_{2} \leq u \leq z_{1}, \\
\left(\frac{z_{2}}{w}\right)^{\Delta} \leq\left(\frac{u}{w}\right)^{\Delta} \leq\left(\frac{z_{1}}{w}\right)^{\Delta} \text { for } x \in\left[a, x^{*}\right]_{\mathbb{V}} .
\end{gathered}
$$

From these, we get the bounds for $u\left(x^{*}\right)$ and $u^{\Delta}\left(x^{*}\right)$. In addition, if a function $w^{*}$ which satisfies $(L+h)\left[w^{*}\right] \leq 0$ on an interval $\left[x^{*}, b\right]_{\mathbb{T}}$ is given, we can then find bounds for $u / w^{*}$ and $\left(u / w^{*}\right)^{\Delta}$, as before. Let the functions $z_{1}^{*}$ and $z_{2}^{*}$ be defined on $\left[x^{*}, b\right]_{\mathbb{T}}$ and they satisfy

$$
\begin{aligned}
& (L+h)\left[z_{2}^{*}\right] \leq f(x) \leq(L+h)\left[z_{1}^{*}\right] \quad \text { in }\left[x^{*}, b\right]_{\mathbb{T}}, \\
& z_{2}^{*}\left(x^{*}\right) \leq u\left(x^{*}\right) \leq z_{1}^{*}\left(x^{*}\right), \\
& \left(\frac{z_{2}^{*}}{w^{*}}\right)^{\Delta} \leq\left(\frac{u}{w^{*}}\right)^{\Delta} \leq\left(\frac{z_{1}^{*}}{w^{*}}\right)^{\Delta} \quad \text { at } x=x^{*} .
\end{aligned}
$$

Then we find, as we did previously, that

$$
\begin{gathered}
z_{2}^{*}(x) \leq u(x) \leq z_{1}^{*}(x) \\
\left(\frac{z_{2}^{*}}{w^{*}}\right)^{\Delta} \leq\left(\frac{u}{w^{*}}\right)^{\Delta} \leq\left(\frac{z_{1}^{*}}{w^{*}}\right)^{\Delta} \text { for } x \in\left[x^{*}, b\right]_{\mathbb{T}} .
\end{gathered}
$$

While we do not know $u\left(x^{*}\right)$ and $\left(u / w^{*}\right)^{\Delta}$ at $x^{*}$, but we know their bounds, therefore, we can give explicit conditions on the values of $z_{1}^{*},\left(z_{1}^{*}\right)^{\Delta}, z_{2}^{*}$, and $\left(z_{2}^{*}\right)^{\Delta}$ at $x^{*}$ which assure that the above inequalities are satisfied.

In fact, since at $x^{*}$ we should have that

$$
\begin{aligned}
\left(\frac{u}{w^{*}}\right)^{\Delta} & =\left(\frac{u}{w} \cdot \frac{w}{w^{*}}\right)^{\Delta} \\
& =\left(\frac{u}{w}\right)^{\Delta} \cdot \frac{w}{w^{*}}+\frac{u^{\sigma}}{w^{\sigma}} \cdot\left(\frac{w}{w^{*}}\right)^{\Delta} \\
& \leq\left(\frac{z_{1}}{w}\right)^{\Delta} \cdot \frac{w}{w^{*}}+\frac{u^{\sigma}}{w^{\sigma}} \cdot\left(\frac{w}{w^{*}}\right)^{\Delta} \\
& =\left(\frac{z_{1}}{w}\right)^{\Delta} \cdot \frac{w}{w^{*}}-\frac{u^{\sigma}}{w^{* \sigma}} \frac{w}{w^{\sigma}}\left(\frac{\left(w^{*}\right)^{\Delta}}{w^{*}}-\frac{w^{\Delta}}{w}\right), \\
\left(\frac{u}{w^{*}}\right)^{\Delta} & =\left(\frac{w}{w} \cdot \frac{z^{*}}{w^{*}}\right)^{\Delta} \cdot \frac{w}{w^{*}}-\frac{u^{\sigma}}{w^{* \sigma}} \frac{w}{w^{\sigma}}\left(\frac{\left(w^{*}\right)^{\Delta}}{w^{*}}-\frac{w^{\Delta}}{w}\right),
\end{aligned}
$$

if $\left(w^{*}\right)^{\Delta} / w^{*} \geq w^{\Delta} / w$, then we can give the conditions on the values of $z_{1}^{*},\left(z_{1}^{*}\right)^{\Delta}, z_{2}^{*}$, and $\left(z_{2}^{*}\right)^{\Delta}$ at $x^{*}$ which assure that inequality (258) holds as follows:

$$
\begin{aligned}
& z_{1}^{*} \geq z_{1}, \\
& \begin{aligned}
w^{*}\left(\frac{z_{1}^{*}}{w^{*}}\right)^{\Delta} \geq & w\left(\frac{z_{1}}{w}\right)^{\Delta}-\frac{w^{*}}{w^{* \sigma}} \frac{w}{w^{\sigma}} \\
& \times\left(\frac{\left(w^{*}\right)^{\Delta}}{w^{*}}-\frac{w^{\Delta}}{w}\right) z_{2}^{* \sigma} \text { at } x=x^{*}, \\
z_{2}^{*} \leq z_{2}, & \\
w^{*}\left(\frac{z_{2}^{*}}{w^{*}}\right)^{\Delta} \leq & w\left(\frac{z_{2}}{w}\right)^{\Delta}-\frac{w^{*}}{w^{* \sigma}} \frac{w}{w^{\sigma}} \\
& \times\left(\frac{\left(w^{*}\right)^{\Delta}}{w^{*}}-\frac{w^{\Delta}}{w}\right) z_{1}^{* \sigma} \text { at } x=x^{*} .
\end{aligned}
\end{aligned}
$$

If $\left(w^{*}\right)^{\Delta} / w^{*} \leq w^{\Delta} / w$, we replace $z_{2}$ by $z_{1}$ in the coefficient of $\left(\left(w^{*}\right)^{\Delta} / w^{*}-w^{\Delta} / w\right)$ in the first row of the inequalities and replace $z_{1}$ by $z_{2}$ in the second row. If these conditions are satisfied, we have the bounds

$$
\begin{aligned}
& z_{2}^{*}(x) \leq u(x) \leq z_{1}^{*}(x), \\
& \left(\frac{z_{2}^{*}}{w^{*}}\right)^{\Delta} \leq\left(\frac{u}{w^{*}}\right)^{\Delta} \leq\left(\frac{z_{1}^{*}}{w^{*}}\right)^{\Delta} .
\end{aligned}
$$


We now consider $w^{*}$ as an extension of $w$ to the interval $\left[x^{*}, b\right]_{\mathbb{T}}, z_{1}^{*}$ as an extension $z_{1}$ to the interval $\left[x^{*}, b\right]_{\mathbb{T}}$, and $z_{2}^{*}$ as an extension of $z_{2}$ to the interval $\left[x^{*}, b\right]_{\mathbb{T}}$. Then these extended functions are, in general, discontinuous at $x^{*}$. However, the above inequalities relating $w, w^{*}, z_{1}^{*}, z_{1}, z_{2}^{*}$, and $z_{2}$ at $x=x^{*}$ establish the relation between the right and left limits at discontinuous points. It may, of course, be necessary or desirable to divide the interval $[a, b]_{\mathbb{T}}$ into more than two subintervals.

The above discussion leads to the following theorem.

Theorem 75. Let $z_{1}(x), z_{2}(x)$, and $w(x)$ be piecewise continuous functions on the interval $[a, b]_{\mathbb{T}}$. Moreover, we assume that $z_{i}^{\Delta}(x), z_{i}^{\Delta \nabla}(x)(i=1,2)$ and $w^{\Delta}(x), w^{\Delta \nabla}(x)$ are piecewise continuous on the interval $[a, b]_{\mathbb{T}}, z_{i}^{\nabla}(x)$ and $w^{\nabla}(x)$ are piecewise left-dense continuous on the interval $[a, b]_{\mathbb{T}}$, and $g_{1}$, $g_{2}$, and $h$ satisfy the conditions of Lemma 54. If the following properties hold:

(a) $w>0$ on $[a, b]_{\mathbb{T}}$;

(b) $z_{2}(a) \leq \gamma_{1} \leq z_{1}(a)$;

(c) $z_{2}^{\Delta}(a) w(a)-z_{2}(a) w^{\Delta}(a) \leq \gamma_{2} w(a)-\gamma_{1} w^{\Delta}(a) \leq$ $z_{1}^{\Delta}(a) w(a)-z_{1}(a) w^{\Delta}(a)$

(d) $(L+h)[w] \leq 0,(L+h)\left[z_{2}\right] \leq f(x) \leq(L+h)\left[z_{1}\right]$ hold at all points where the derivatives occurring in these formulas are continuous;

(e) at each point of discontinuity $x^{*}$ the functions $z_{1}$, $-z_{2}$, and $w^{\Delta} / w$ have nonnegative jumps, the jump of $w\left(z_{1} / w\right)^{\Delta}$ is at least $-\left(\left(w^{*} / w^{* \sigma}\right)\left(w / w^{\sigma}\right)\right) z_{2}^{* \sigma}$ times as many as jump of $w^{\Delta} / w$, and the jump of $w\left(z_{2} / w\right)^{\Delta}$ is at most $-\left(\left(w^{*} / w^{* \sigma}\right)\left(w / w^{\sigma}\right)\right) z_{1}^{* \sigma}$ times as many as jump of $w^{\Delta} / w$

then

$$
\begin{gathered}
\frac{z_{2}}{w} \leq \frac{u}{w} \leq \frac{z_{1}}{w} \\
\left(\frac{z_{2}}{w}\right)^{\Delta} \leq\left(\frac{u}{w}\right)^{\Delta} \leq\left(\frac{z_{1}}{w}\right)^{\Delta} \text { on }[a, b]_{\mathbb{T}} .
\end{gathered}
$$

\section{Applications to Boundary Value Problems}

In this section, by using the maximum principles proved in Section 3 to some general boundary value problems, the uniqueness of the solutions, the existence of the upper and lower solutions, and some necessary and sufficient conditions for the existence of the approximation solutions are discussed. First, we consider the following boundary value problems:

$$
\begin{gathered}
u^{\Delta \nabla}+g_{1} u^{\Delta}+g_{2} u^{\nabla}+h u=f(x), \quad \text { on }(a, b)_{\mathbb{T}}, \\
u(a)=\gamma_{1}, \quad u(b)=\gamma_{2} .
\end{gathered}
$$

Theorem 76. Assume that $g_{1}, g_{2}$, and h are bounded on $(a, b)_{\mathbb{T}}$ such that (91), (92), (93), and (130) hold and $h(x) \leq 0$ at each $x \in(a, b)_{\mathbb{T}}$. If $u_{1}, u_{2} \in \mathscr{D}(\Lambda)$ are solutions of (264) and satisfy the boundary value problem (265), then $u_{1} \equiv u_{2}$.
Proof. We define a function $v(x) \in \mathscr{D}(\Lambda)$ by

$$
v(x)=u_{1}(x)-u_{2}(x) .
$$

Since both $u_{1}$ and $u_{2}$ satisfy (264) and (265), the function $v(x)$ satisfies

$$
\begin{aligned}
& v^{\Delta \nabla}+g_{1} v^{\Delta}+g_{2} u^{\nabla}+h v=0, \\
& v(a)=0, \quad v(b)=0 .
\end{aligned}
$$

It follows from Theorem 47 that $v(x) \leq 0$, for each $x \in(a, b)_{\mathbb{T}}$. Since $-v(x)$ satisfies the same boundary value problem, we have $-v(x) \leq 0$, for each $x \in(a, b)_{\mathbb{T}}$, and thus $v(x) \equiv 0$, for each $x \in[a, b]_{\mathbb{T}}$. form

Next we study general boundary value problems of the

$$
\begin{gathered}
u^{\Delta \nabla}+g_{1} u^{\Delta}+g_{2} u^{\nabla}+h u=f(x), \\
-u^{\Delta}(a) \cos \theta+u(a) \sin \theta=\gamma_{1}, \\
u^{\nabla}(b) \cos \phi+u(b) \sin \phi=\gamma_{2},
\end{gathered}
$$

where $\theta, \phi, \gamma_{1}, \gamma_{2}$ are all constant, and $0 \leq \theta \leq \pi / 2,0 \leq \phi \leq$ $\pi / 2$. If $\theta=\phi=\pi / 2$, (269) becomes (265).

Theorem 77. Assume that $g_{1}, g_{2}$, and h are bounded on $(a, b)_{\mathbb{T}}$ such that (91), (92), (93), and (130) hold. If $u_{1}$ and $u_{2}$ are solutions of (268) and (269), then $u_{1} \equiv u_{2}$, except that $u_{1}(x)-$ $u_{2}(x)$ is a constant when $h(x) \equiv 0, \theta=\phi=0$.

Proof. We define a function $v(x) \in \mathscr{D}(\Lambda)$ by

$$
v(x)=u_{1}(x)-u_{2}(x) .
$$

Since both $u_{1}$ and $u_{2}$ satisfy (268) and (269), the function $v(x)$ satisfies

$$
\begin{aligned}
& v^{\Delta \nabla}+g_{1} v^{\Delta}+g_{2} u^{\nabla}+h v=0, \\
& -v^{\Delta}(a) \cos \theta+v(a) \sin \theta=0, \\
& v^{\nabla}(b) \cos \phi+v(b) \sin \phi=0 .
\end{aligned}
$$

It is clear that $v(x) \equiv C$ satisfies all the above conditions, if and only if $h(x) \equiv 0, \theta=\phi=0$. Then we assume first that $v(x)>0$ at some point and $v(x)$ is not constant. Using Theorem 47 we know that $v(x)$ attains its maximum $M$ at $a$ or $b$. Suppose that $v(a)=M$, and by using Theorem 51 we get $v^{\Delta}(a)<0$, which do not satisfy (272). Suppose that $v(b)=M$, and by using Theorem 51 we get $v^{\nabla}(b)>0$, which do not satisfy (273). Thus, we obtain $v(x) \leq 0$. We can also prove that $-v(x) \leq 0$, and then $v(x) \equiv 0$, for each $x \in[a, b]_{\mathbb{T}}$.

Similar to the initial value problems, in most cases it is impossible to find such a solution explicitly. But, it is frequently desirable to approximate a solution in such a way that an explicit bound for the error is known. Such an approximation is equivalent to the determination of both upper and lower bounds for the values of the solution. Thus, 
in the following, we will discuss the existence of the upper and lower solutions for boundary value problems.

We will assume that the functions $f, g_{1}, g_{2}, h$, are bounded and $h(x) \leq 0$ in $[a, b]_{\mathbb{T}}$. Under these circumstances it is possible to use the maximum principle in Theorem 55 to obtain a bound for a solution $u$ without any actual knowledge of $u$ itself.

Suppose we can find a function $z_{1}(x) \in \mathscr{D}(\Lambda)$ satisfying

$$
\begin{gathered}
(L+h)\left[z_{1}\right] \leq f(x), \\
z_{1}(a) \geq \gamma_{1}, \quad z_{1}(b) \geq \gamma_{2} .
\end{gathered}
$$

Then the function

$$
v_{1}(x) \equiv u(x)-z_{1}(x)
$$

satisfies

$$
\begin{gathered}
(L+h)\left[v_{1}\right] \geq 0, \\
v_{1}(a) \leq 0, \quad v_{1}(b) \leq 0 .
\end{gathered}
$$

The maximum principles as given in Theorem 47 in Section 3 may be applied to $v_{1}$, and we conclude that $v_{1}(x) \leq 0$ on $[a, b]_{\mathbb{T}}$. That is,

$$
u(x) \leq z_{1}(x) \text { for } x \in[a, b]_{\mathbb{T}} .
$$

The function $z_{1}(x)$ is an upper bound for $u(x)$.

Similarly, a lower bound for $u(x)$ may be obtained by finding a function $z_{2}(x)$ with the properties

$$
\begin{gathered}
(L+h)\left[z_{2}\right] \geq f(x), \\
z_{2}(a) \leq \gamma_{1}, \quad z_{2}(b) \leq \gamma_{2} .
\end{gathered}
$$

By using the maximum principle (Theorem 51) to $z_{2}(x)-u(x)$, we can get that

$$
u(x) \geq z_{2}(x) \text { for } x \in[a, b]_{\mathbb{T}} .
$$

Functions $z_{1}(x), z_{2}(x)$ with the desired properties are easily constructed. For example, we may set

$$
z_{1}(x)=A\left(2-\widehat{e}_{\alpha}(a, x)\right)
$$

with $\alpha<0$ and try to select $A$ and $\alpha$ so that (274) and (275) are satisfied. In fact, if (166) holds, we can choose $-\alpha$ to be so large that

$$
\begin{aligned}
(L+h)\left[\widehat{e}_{\alpha}(a, x)\right] & \\
= & {\left[\alpha^{2}\left(1+\nu g_{1}\right)-\alpha\left(g_{1}+g_{2}\right)+h(1-\alpha \nu(x))\right] } \\
& \times \frac{\widehat{e}_{\alpha}(a, x)}{1-\alpha \nu(x)}>0
\end{aligned}
$$

for $x \in[a, b]_{\mathbb{T}}$. Let

$$
\begin{aligned}
k=\min _{x \in[a, b]_{\mathbb{T}}}[ & {\left[\alpha^{2}\left(1+\nu g_{1}\right)-\alpha\left(g_{1}+g_{2}\right)+h(1-\alpha \nu(x))\right] } \\
& \left.\times \frac{\widehat{e}_{\alpha}(a, x)}{1-\alpha \nu(x)}\right]
\end{aligned}
$$

for $x \in[a, b]_{\mathbb{T}}$, and

$$
A=\max \left\{\gamma_{1}, \gamma_{2}, \frac{1}{k} \max _{x \in[a, b]_{\mathbb{T}}}\{-f(x)\}, 0\right\} .
$$

For the selections of $A$ and $\alpha$ as just described, the function $z_{1}(x)=A\left(l-\widehat{e}_{\alpha}(a, x)\right)$ satisfies (274) and (275), where $l=$ $1+\widehat{e}_{\alpha}(a, b)$.

To determine a lower bound, we choose

$$
z_{2}(x)=B\left(l-\widehat{e}_{\alpha}(a, x)\right),
$$

with $\alpha<0$, and try to select $B$ and $\alpha$ such that (279) and (280) are satisfied. In fact, we choose $-\alpha$ to be so large that (283) holds. Let

$$
B=\min \left\{\gamma_{1}, \gamma_{2},-\frac{1}{k} \max _{x \in[a, b]_{\mathbb{T}}}\{f(x)\}, 0\right\} .
$$

With the selections of $B$ and $\alpha$ as just described, the function $z_{2}(x)=B\left(l-\widehat{e}_{\alpha}(a, x)\right)$ satisfies (279) and (280). Then

$$
B\left(l-\widehat{e}_{\alpha}(a, x)\right) \leq u(x) \leq A\left(l-\widehat{e}_{\alpha}(a, x)\right) .
$$

In particular, we have

$$
|u(x)| \leq l \max \left[\left|\gamma_{1}\right|,\left|\gamma_{2}\right|, \frac{1}{k} \max _{x \in[a, b]_{\mathbb{T}}}|f(x)|, 0\right] .
$$

If $u$ is a solution of (264) and (265) and $\bar{u}$ is a solution of the related problem

$$
\begin{aligned}
& \bar{u}^{\Delta \nabla}+g_{1} \bar{u}^{\Delta}+g_{2} \bar{u}^{\nabla}+h \bar{u}=\bar{f}(x), \\
& \bar{u}(a)=\overline{\gamma_{1}}, \quad \bar{u}(b)=\overline{\gamma_{2}},
\end{aligned}
$$

then the difference $u-\bar{u}$ satisfies

$$
\begin{aligned}
& (u-\bar{u})^{\Delta \nabla}+g_{1}(u-\bar{u})^{\Delta}+g_{2}(u-\bar{u})^{\nabla} \\
& \quad+h(u-\bar{u})=f-\bar{f}, \\
& u(a)-\bar{u}(a)=\gamma_{1}-\overline{\gamma_{1}}, \quad u(b)-\bar{u}(b)=\gamma_{2}-\overline{\gamma_{2}} .
\end{aligned}
$$

Inequality (289) shows that

$$
\begin{aligned}
|u(x)-\bar{u}(x)| & \\
\leq l \max [ & \left|\gamma_{1}-\overline{\gamma_{1}}\right|,\left|\gamma_{2}-\overline{\gamma_{2}}\right|, \\
& \left.\frac{1}{k} \max _{x \in[a, b]_{\pi}}|f(x)-\bar{f}(x)|\right] .
\end{aligned}
$$

Therefore, if the quantities

$$
\left[\left|\gamma_{1}-\overline{\gamma_{1}}\right|,\left|\gamma_{2}-\overline{\gamma_{2}}\right|, \max _{x \in[a, b]_{\pi}}|f(x)-\bar{f}(x)|\right]
$$

are all small, then $|u(x)-\bar{u}(x)|$ is small for all $x$ in the interval $[a, b]_{\mathbb{T}}$. Under these circumstances, we say that the solution $u$ of the problem (264), (265) depends continuously on $f(x)$ and the boundary values $\gamma_{1}, \gamma_{2}$.

Combining the above discussions, we get the following result. 
Theorem 78. If $g_{1}, g_{2}$, and $h$ are bounded on $(a, b)_{\mathbb{T}}$ such that (92), (93), (130), and (166) hold and $h(x) \leq 0$ in $[a, b]_{\mathbb{T}}$, then the following conclusions hold:

(1) there exist functions $z_{1}(x), z_{2}(x) \in \mathscr{D}(\Lambda)$ satisfying (274), (275) and (279), (280), respectively;

(2) the solution $u$ of the problem (264), (265) satisfies $z_{1}(x) \leq u(x) \leq z_{2}(x), x \in[a, b]_{\mathbb{T}} ;$

(3) the solution $u$ of the problem (264), (265) depends continuously on $f(x)$ and the boundary values $\gamma_{1}, \gamma_{2}$.

Next, we consider the question of approximations of solutions of (268) and (269).

If we can find a function $z_{1}(x) \in \mathscr{D}(\Lambda)$ satisfying

$$
\begin{gathered}
(L+h)\left[z_{1}\right] \leq f(x), \\
-z_{1}^{\Delta}(a) \cos \theta+z_{1}(a) \sin \theta \geq \gamma_{1}, \\
z_{1}^{\nabla}(b) \cos \phi+z_{1}(b) \sin \phi \geq \gamma_{2},
\end{gathered}
$$

we define a function $v_{1}(x) \in \mathscr{D}(\Lambda)$ by

$$
v_{1}(x) \equiv u(x)-z_{1}(x) .
$$

Thus,

$$
\begin{gathered}
(L+h)\left[v_{1}\right] \geq 0, \\
-v_{1}^{\Delta}(a) \cos \theta+v_{1}(a) \sin \theta \leq 0, \\
v_{1}^{\nabla}(b) \cos \phi+v_{1}(b) \sin \phi \leq 0 .
\end{gathered}
$$

It follows from Theorem 51, (297) we know that $v_{1}(x)$ cannot attain its maximum at $a$ or $b$. If $v_{1}(x)>0$ at some point $x \in(a, b)_{\mathbb{T}}$, then by using Theorem 47 , we have that $v_{1}(x)$ is positive constant which implies that $\theta=\phi=0$ and $h(x) \equiv 0$. Otherwise, if $h(x) \equiv 0, \theta=0, \phi=0$ are not all hold, then we have that $v_{1}(x) \leq 0$, that is $u(x) \leq z_{1}(x)$. Similarly, we assume that we can find a function $z_{2}(x) \in \mathscr{D}(\Lambda)$ satisfying:

$$
\begin{gathered}
(L+h)\left[z_{2}\right] \geq f(x), \\
-z_{2}^{\Delta}(a) \cos \theta+z_{2}(a) \sin \theta \leq \gamma_{1}, \\
z_{2}^{\nabla}(b) \cos \phi+z_{2}(b) \sin \phi \leq \gamma_{2} .
\end{gathered}
$$

The same as the above statement, we define

$$
v_{2}(x) \equiv z_{2}(x)-u(x),
$$

and we obtain

$$
u(x) \geq z_{2}(x) .
$$

Therefore, we establish an approximation theorem as in the following.

Theorem 79. Assume that $g_{1}, g_{2}$, and h are bounded on $(a, b)_{\mathbb{T}}$ and satisfy (91), (92), (93), and (130) at each $x \in(a, b)_{\mathbb{T}}$. If $u(x)$ is a solution of (268) and (269), where $h(x) \leq 0,0 \leq \theta \leq \pi / 2$, $0 \leq \phi \leq \pi / 2$. Let $z_{1}(x)$ and $z_{2}(x) \in \mathscr{D}(\Lambda)$ satisfy (294), (295), (298), and (299). If $h(x) \equiv 0, \theta=0, \phi=0$ are not all hold, then $z_{2}(x) \leq u(x) \leq z_{1}(x)$.
Now, we consider deleting the conditions $h(x) \leq 0,0 \leq$ $\theta \leq \pi / 2,0 \leq \phi \leq \pi / 2$ in Theorem 79. Without loss of generality, we can assume that $-\pi / 2 \leq \theta \leq \pi / 2,-\pi / 2 \leq \phi \leq$ $\pi / 2$.

In order to use the generalized maximum principle established in Section 3, we suppose that there is a positive function $w(x)$ which satisfies the inequalities

$$
\begin{gathered}
(L+h)[w] \leq 0 \quad \text { in }[a, b]_{\mathbb{T}}, \\
-w^{\Delta}(a) \cos \theta+w(a) \sin \theta \geq 0, \\
w^{\nabla}(b) \cos \phi+w(b) \sin \phi \geq 0 .
\end{gathered}
$$

We set

$$
v=\frac{u}{w}
$$

where $u(x)$ is a solution of (268) and (269). Then $v$ must satisfy

$$
(\bar{L}+H)[v]=v^{\Delta \nabla}+G_{1} v^{\Delta}+G_{2} v^{\nabla}+H v=\frac{f}{w^{\sigma \rho}}
$$

with $G_{1}=\left(F^{\Delta \nabla}+g_{1} w^{\sigma}\right) / w^{\sigma \rho}, G_{2}=\left(w^{\Delta \rho}+g_{2} w^{\rho}\right) / w^{\sigma \rho}, H=$ $\left(1 / w^{\sigma \rho}\right)(L+h)[w] \leq 0$, and

$$
\begin{gathered}
-\left[w^{\Delta}(a) v(a)+w(\sigma(a)) v^{\Delta}(a)\right] \cos \theta \\
+v(a) w(a) \sin \theta=\gamma_{1}, \\
{\left[w^{\nabla}(b) v(b)+w(\rho(b)) v^{\nabla}(b)\right] \cos \phi} \\
+v(b) w(b) \sin \phi=\gamma_{2} .
\end{gathered}
$$

We can rewrite (306) to be

$$
\begin{aligned}
& -v^{\Delta}(a) \cos \tilde{\theta}+v(a) \sin \tilde{\theta}=\widetilde{\gamma_{1}}, \\
& v^{\nabla}(b) \cos \widetilde{\phi}+v(b) \sin \widetilde{\phi}=\widetilde{\gamma_{2}},
\end{aligned}
$$

where

$$
\begin{aligned}
& \tan \tilde{\theta}=\frac{-w^{\Delta}(a) \cos \theta+w(a) \sin \theta}{w(\sigma(a)) \cos \theta} \geq 0, \\
& \tan \tilde{\phi}=\frac{w^{\nabla}(b) \cos \phi+w(b) \sin \phi}{w(\rho(b)) \cos \phi} \geq 0, \\
& \widetilde{\gamma_{1}}=\frac{\gamma_{1}}{\sqrt{\left[w(a) \sin \theta-w^{\Delta}(a) \cos \theta\right]^{2}+[w(\sigma(a)) \cos \theta]^{2}}}, \\
& \widetilde{\gamma_{2}}=\frac{\gamma_{2}}{\sqrt{\left[w^{\nabla}(b) \cos \phi+w(b) \sin \phi\right]^{2}+[w(\rho(b)) \cos \phi]^{2}}} .
\end{aligned}
$$

By (303) we may choose $\widetilde{\theta}$ and $\widetilde{\phi}$ in the range $0 \leq \widetilde{\theta} \leq \pi / 2$, $0 \leq \tilde{\phi} \leq \pi / 2$.

Suppose that there is a function $w(x)$, which is positive on $[a, b]_{\mathbb{T}}$ and satisfies conditions (302) and (303). If $z_{1}(x)$ and 
$z_{2}(x)$ satisfy (294), (295) and (298), (299), respectively, then the functions

$$
\frac{z_{1}}{w}, \quad \frac{z_{2}}{w}
$$

satisfy the analogous conditions with respect to (305) with boundary (307); that is, the following inequalities hold:

$$
\begin{gathered}
(\bar{L}+H)\left[\frac{z_{1}}{w}\right] \leq \frac{f}{w^{\sigma \rho}}, \\
-\left[\frac{z_{1}}{w}\right]^{\Delta}(a) \cos \tilde{\theta}+\frac{z_{1}}{w}(a) \sin \tilde{\theta} \geq \tilde{\gamma}_{1}, \\
{\left[\frac{z_{1}}{w}\right]^{\nabla}(b) \cos \tilde{\phi}+\frac{z_{1}}{w}(b) \sin \tilde{\phi} \geq \tilde{\gamma}_{2},} \\
-\left[\frac{z_{2}}{w}\right]^{\Delta}(a) \cos \tilde{\theta}+\frac{z_{2}}{w}(a) \sin \tilde{\theta} \leq \tilde{\gamma}_{1}, \\
{\left[\frac{z_{2}}{w}\right]^{\nabla}(b) \cos \tilde{\phi}+\frac{z_{2}}{w}(b) \sin \phi \leq \tilde{\gamma}_{2} .}
\end{gathered}
$$

Hence, by Theorem 79, we have the inequalities

$$
\frac{z_{2}}{w} \leq \frac{u}{w} \leq \frac{z_{1}}{w}
$$

unless $\widetilde{\theta}=\widetilde{\phi}=0$ and $H(x) \equiv 0$.

If $w(x)$ satisfies (302) and (303) with equality rather than inequality, we may add a multiple of $w$ to a solution $u$ of (268) and (269) to obtain another solution. That is, the solution is not unique. Of course, there may be no solution at all, but if there is at least one, then there are many. Therefore, if there is a positive function $w(x)$ that satisfies (302) and (303) but such that not all the inequalities are equations, we obtain the bounds

$$
z_{2}(x) \leq u(x) \leq z_{1}(x)
$$

as before.

If inequality (312) holds for the solution of (268) and (269), then, particularly, the solution $w$ of

$$
(L+h)[w]=0 \quad \text { in }(a, b)_{\mathbb{T}},
$$

which satisfies the boundary conditions

$$
\begin{aligned}
& -w^{\Delta}(a) \cos \theta+w(a) \sin \theta=1, \\
& w^{\nabla}(b) \cos \phi+w(b) \sin \phi=1,
\end{aligned}
$$

must be nonnegative.

In fact, if we select $z_{2}(x) \equiv 0$, then with respect to (313) and (314), $z_{2}(x)$ satisfies (298) and (299) for $f(x) \equiv 0, \gamma_{1}=$ $\gamma_{2}=1$. Then we have that $z_{2}(x) \leq w(x)$, that is, $w(x) \geq 0$. Moreover, if $w\left(x_{0}\right)=0, x_{0}$ in $(a, b)_{\mathbb{T}}$, then $-w\left(x_{0}\right)=0$ is a maximum of $-w$ in $(a, b)_{\mathbb{T}}$; by using Lemma 34 to $-w$ we obtain

$$
w^{\Delta}\left(x_{0}\right) \geq 0, \quad w^{\nabla}\left(x_{0}\right) \leq 0 .
$$

If $x_{0}$ is left-scattered, since

$$
(L+h)[w]=0
$$

we have

$$
\begin{aligned}
(L & +h)\left[w\left(x_{0}\right)\right] \\
& =w^{\Delta \nabla}\left(x_{0}\right)+g_{1} w^{\Delta}\left(x_{0}\right)+g_{2} w^{\nabla}\left(x_{0}\right)+h w\left(x_{0}\right) \\
& =\frac{w^{\Delta}\left(x_{0}\right)-w^{\nabla}\left(x_{0}\right)}{\nu\left(x_{0}\right)}+g_{1} w^{\Delta}\left(x_{0}\right)+g_{2} w^{\nabla}\left(x_{0}\right) \\
& =0
\end{aligned}
$$

and hence

$$
\left(1+v\left(x_{0}\right) g_{1}\right) w^{\Delta}\left(x_{0}\right)=-\left(-1+v\left(x_{0}\right) g_{2}\right) w^{\nabla}\left(x_{0}\right)
$$

by (166), (92), and (315), we obtain $w^{\Delta}\left(x_{0}\right)=0$ (if $-1+$ $v\left(x_{0}\right) g_{2}<0$, we can also have that $\left.w^{\nabla}\left(x_{0}\right)=0\right)$. If $x_{0}$ is leftdense, by Lemma 34, we get that

$$
\begin{gathered}
w^{\Delta}\left(x_{0}\right)=0 \\
w^{\nabla}\left(x_{0}\right)=w^{\Delta}\left(\rho\left(x_{0}\right)\right)=w^{\Delta}\left(x_{0}\right)=0 .
\end{gathered}
$$

So we have proved that $w^{\Delta}\left(x_{0}\right)=0$ if $w\left(x_{0}\right)=0$. The uniqueness theorem for the initial value problem implies that $w \equiv 0$, which violates the boundary conditions (314). So $w$ cannot vanish in $(a, b)_{\mathbb{T}}$. If $w$ vanishes at endpoint, say at $a$, then the first condition in (314) becomes $-w^{\Delta}(a) \cos \theta=1$, which is a contradiction. Therefore $w(a)>0$ and, similarly, $w(b)>0$. Hence $w>0$ on $[a, b]_{\mathbb{T}}$.

Thus far, under the hypothesis that the problem (313), (314) has a solution, we have established the following result.

Theorem 80. Let $u$ be a solution of (268), (269) with $-\pi / 2 \leq$ $\theta \leq \pi / 2,-\pi / 2 \leq \phi \leq \pi / 2$. Let $z_{1}(x)$ and $z_{2}(x) \in \mathscr{D}(\Lambda)$ satisfy (294), (295) and (298), (299), respectively; $g_{1}, g_{2}$, and $h$ are bounded on $(a, b)_{\mathbb{T}}$ and satisfy (91), (92). If problem (313), (314) has a solution, then the bounds

$$
z_{2}(x) \leq u(x) \leq z_{1}(x)
$$

hold in $(a, b)_{\mathbb{T}}$ if and only if there exists a positive function $w$ on $[a, b]_{\mathbb{T}}$ which satisfies inequalities (302) and (303) in such a way that not all the inequalities in (302) and (303) are equalities.

Remark 81. Moreover, if $h(x) \leq 0,0 \leq \theta \leq \pi / 2$, and $0 \leq \phi \leq$ $\pi / 2$, then the function $w \equiv 1$ satisfies conditions (302) and (303); thus Theorem 79 can be deduced from Theorem 80 .

The function $w$ does not appear in the inequality (320). Therefore, it is of interest to obtain a theorem which eliminates $w$ entirely and which provides conditions on $z_{1}$ and $z_{2}$ guaranteeing that they form the upper and lower bounds of the solution of (268), (269). The next result gives a necessary and sufficient condition for this case. 
Theorem 82. Let $u$ be a solution of (268), (269) with $-\pi / 2<$ $\theta<\pi / 2,-\pi / 2<\phi<\pi / 2$. Suppose $z_{1}(x)$ and $z_{2}(x) \in$ $\mathscr{D}(\Lambda)$ satisfy (294), (295) and (298), (299), respectively, and in all these inequalities, at least there is one which is a strict inequality. Let $g_{1}, g_{2}$ be bounded and satisfy $1+v g_{1}>0$, $-1+v g_{2}<0$. Then the bounds

$$
z_{2}(x) \leq u(x) \leq z_{1}(x)
$$

hold if and only if $z_{2}(x) \leq z_{1}(x)$ for $x \in[a, b]_{\mathbb{T}}$.

Proof. If (321) holds, then it is clear that $z_{2}(x) \leq z_{1}(x)$. We now assume that $z_{1}(x)-z_{2}(x)$ is nonnegative, and we must show that (321) holds. If

$$
q(x) \equiv z_{1}(x)-z_{2}(x)
$$

is strictly positive on $[a, b]_{\mathbb{T}}$, then we may select $q$ as the function $w$ of Theorem 80 . All the requirements are fulfilled and Theorem 80 implies that (321) holds. Therefore, we need only to investigate the possibility that $q$ has a zero point on $[a, b]_{\mathbb{T}}$.

According to (294), (295), (298), and (299), $q$ satisfies the inequalities

$$
\begin{gathered}
(L+h)[q] \leq 0 \quad \text { in }[a, b]_{\mathbb{T}}, \\
-q^{\Delta}(a) \cos \theta+q(a) \sin \theta \geq 0, \\
q^{\nabla}(b) \cos \phi+q(b) \sin \phi \geq 0,
\end{gathered}
$$

and, in view of the hypotheses, at least there is one which is a strict inequality in (323), (324).

First suppose $q(c)=0, c \in(a, b)_{\mathbb{T}}$. Then $q$ has a minimum at $c$, which shows that $-q$ has a maximum at $c$. So by Lemma 34 we obtain

$$
q^{\Delta}(c) \geq 0, \quad q^{\nabla}(c) \leq 0 .
$$

If $c$ is left-scattered, since

$$
(L+h)[q] \leq 0
$$

we have

$$
\begin{aligned}
(L & +h)[q(c)] \\
& =q^{\Delta \nabla}(c)+g_{1} q^{\Delta}(c)+g_{2} q^{\nabla}(c)+h q(c) \\
& =\frac{q^{\Delta}(c)-q^{\nabla}(c)}{\nu(c)}+g_{1} q^{\Delta}(c)+g_{2} q^{\nabla}(c) \\
& \leq 0
\end{aligned}
$$

and hence

$$
0 \leq\left(1+\nu g_{1}\right) q^{\Delta}(c) \leq-\left(-1+v(x) g_{2}\right) q^{\nabla}(c) \leq 0 .
$$

Therefore

$$
q^{\Delta}(c)=q^{\nabla}(c)=0
$$

If $c$ is left-dense by Lemma 34, we have that

$$
q^{\nabla}(c)=q^{\Delta}(c)=0 .
$$

And we conclude from Theorem 68 that $q \equiv 0$. Then equality holds in all the conditions (323), (324) contrary to our hypotheses.

The only remaining possibility is that $q>0$ in $(a, b)_{\mathbb{T}}$ but that $q=0$ at an endpoint, say at $x=a$. Then according to Theorem 55, $q^{\Delta}(a)>0$. But then the first inequality in (324) is violated unless $\theta= \pm \pi / 2$, which is a contradiction with $\theta \neq \pm \pi / 2$. Similarly, if $q$ vanishes at $b$, then $\phi= \pm \pi / 2$, which is a contradiction with $\phi \neq \pm \pi / 2$. Therefore, we have proved that $q$ is positive on $[a, b]_{\mathbb{T}}$, and it can be used as an auxiliary function in Theorem 80 . The proof is completed.

\section{Applications to Nonlinear Operator}

In this section, we discuss nonlinear equations. We can extend the results of linear operator in Sections 4 and 5 to nonlinear operator.

Suppose that $u(x) \in \mathscr{D}(\Lambda)$ is a solution of

$$
u^{\Delta \nabla}+H\left(x, u, u^{\Delta}, u^{\nabla}\right)=0 .
$$

Assume that

$$
\begin{array}{ll}
H(x, y, z, p), & \frac{\partial H(x, y, z, p)}{\partial y} \\
\frac{\partial H(x, y, z, p)}{\partial z}, & \frac{\partial H(x, y, z, p)}{\partial p}
\end{array}
$$

are all continuous functions in their domain and satisfy

$$
1+v \frac{\partial H(x, y, z, p)}{\partial z}>0, \quad-1+v \frac{\partial H(x, y, z, p)}{\partial p}<0
$$

where $H(x, y, z, p)$ is defined on $\mathbb{T} \times \mathbb{R} \times \mathbb{R} \times \mathbb{R}$. Besides, we suppose that

$$
H\left(x, y_{1}, z, p\right) \leq H\left(x, y_{2}, z, p\right) \quad \text { when } y_{1} \geq y_{2}
$$

for each $x, z, p$, or equivalently

$$
\frac{\partial H(x, y, z, p)}{\partial y} \leq 0
$$

Remark 83. Note that if

$$
H\left(x, u, u^{\Delta}, u^{\nabla}\right)=g_{1} u^{\Delta}+g_{2} u^{\nabla}+h u-f(x),
$$

then (331) is the linear equation considered in the previous sections.

Assume that $w(x) \in \mathscr{D}(\Lambda)$ satisfies

$$
w^{\Delta \nabla}+H\left(x, w, w^{\Delta}, w^{\nabla}\right) \geq 0
$$


We define a function $v(x) \in \mathscr{D}(\Lambda)$ by

$$
v(x)=w(x)-u(x) .
$$

Subtracting (331) from (337), we derive that

$$
v^{\Delta \nabla}+H\left(x, w, w^{\Delta}, w^{\nabla}\right)-H\left(x, u, u^{\Delta}, u^{\nabla}\right) \geq 0 .
$$

Applying mean value theorem to $H$, we obtain

$$
\begin{aligned}
v^{\Delta \nabla}+ & \frac{\partial H(x, y, z, p)}{\partial z} v^{\Delta}+\frac{\partial H(x, y, z, p)}{\partial p} v^{\nabla} \\
+ & \frac{\partial H(x, y, z, p)}{\partial y} v \geq 0,
\end{aligned}
$$

where $\quad \partial H(x, y, z, p) / \partial y, \quad \partial H(x, y, z, p) / \partial z, \quad$ and $\partial H(x, y, z, p) / \partial p$ are calculated at the point of $(x, u+\alpha(w-u)$, $\left.u^{\Delta}+\alpha\left(w^{\Delta}-u^{\Delta}\right), u^{\nabla}+\alpha\left(w^{\nabla}-u^{\nabla}\right)\right)$, and $0<\alpha<1$. Obviously, the function $v(x)$ satisfies a linear equation. We can use Theorems 47 and 72 to get the following results.

Theorem 84. Assume that $w(x), u(x) \in \mathscr{D}(\Lambda)$ satisfy

$$
w^{\Delta \nabla}+H\left(x, w, w^{\Delta}, w^{\nabla}\right) \geq u^{\Delta \nabla}+H\left(x, u, u^{\Delta}, u^{\nabla}\right),
$$

where $H, \partial H / \partial y, \partial H / \partial z, \partial H / \partial p$, are all continuous functions such that (333) and (335) hold and

$$
\begin{gathered}
\frac{\partial H(x, y, z, p) / \partial y}{1+v(\partial H(x, y, z, p) / \partial z)} \\
\frac{\partial H(x, y, z, p) / \partial y+\partial H(x, y, z, p) / \partial p}{1+v(\partial H(x, y, z, p) / \partial z)}
\end{gathered}
$$

are bounded on $\Lambda \times w(\Lambda) \times w^{\Delta}(\Lambda) \times w^{\nabla}(\Lambda)$. If $w(x)-u(x)$ attains its maximum $M$ in $(a, b)_{\mathbb{T}}$, then $w(x)-u(x) \equiv M$.

Theorem 85. Assume that $u(x) \in \mathscr{D}(\Lambda)$ is a solution of

$$
\begin{gathered}
u^{\Delta \nabla}+H\left(x, u, u^{\Delta}, u^{\nabla}\right)=0 \quad x \in(a, b)_{\mathbb{T}}, \\
u(a)=\gamma_{1}, \quad u^{\Delta}(a)=\gamma_{2},
\end{gathered}
$$

where $H, \partial H / \partial y, \partial H / \partial z$, and $\partial H / \partial p$ are all continuous functions such that (333) and (335) hold and

$$
\begin{gathered}
\frac{\partial H(x, y, z, p) / \partial y}{1+\nu(\partial H(x, y, z, p) / \partial z)} \\
\frac{\partial H(x, y, z, p) / \partial y+\partial H(x, y, z, p) / \partial p}{1+v(\partial H(x, y, z, p) / \partial z)}
\end{gathered}
$$

are bounded on $\Lambda \times w(\Lambda) \times w^{\Delta}(\Lambda) \times w^{\nabla}(\Lambda)$. If a function $z_{1}(x)$ satisfies

$$
\begin{aligned}
& z_{1}^{\Delta \nabla}+H\left(x, z_{1}, z_{1}^{\Delta}, z_{1}^{\nabla}\right) \geq 0, \\
& z_{1}(a) \geq \gamma_{1}, \quad z_{1}^{\Delta}(a) \geq \gamma_{2},
\end{aligned}
$$

and a function $z_{2}(x) \in \mathscr{D}(\Lambda)$ satisfies

$$
\begin{aligned}
& z_{2}^{\Delta \nabla}+H\left(x, z_{2}, z_{2}^{\Delta}, z_{2}^{\nabla}\right) \leq 0, \\
& z_{2}(a) \leq \gamma_{1}, \quad z_{2}^{\Delta}(a) \leq \gamma_{2},
\end{aligned}
$$

then we have

$$
z_{2}(x) \leq u(x) \leq z_{1}(x), \quad z_{2}^{\Delta}(x) \leq u^{\Delta}(x) \leq z_{1}^{\Delta}(x) .
$$

Remark 86. Theorem 85 implies that the solution of (343), (344) is unique. In fact, if $u_{1}$ and $u_{2}$ are solutions of (343), (344), we just need to let $z_{2}(x)=z_{1}(x)=u_{2}$, and then we can obtain $u_{1} \equiv u_{2}$.

By using Theorem 79, we have the following result.

Theorem 87. Assume that $u(x) \in \mathscr{D}(\Lambda)$ is a solution of

$$
\begin{gathered}
u^{\Delta \nabla}+H\left(x, u, u^{\Delta}, u^{\nabla}\right)=0 \quad x \in(a, b)_{\mathbb{T}}, \\
-u^{\Delta}(a) \cos \theta+u(a) \sin \theta=\gamma_{1}, \\
u^{\nabla}(b) \cos \phi+u(b) \sin \phi=\gamma_{2},
\end{gathered}
$$

where $0 \leq \theta \leq \pi / 2,0 \leq \phi \leq \pi / 2$, and $\theta$, $\phi$ are not all equal to $0, H, \partial H / \partial y, \partial H / \partial z$, and $\partial H / \partial p$ are all continuous functions such that (333) and (335) hold, and

$$
\begin{aligned}
& \frac{\partial H(x, y, z, p) / \partial y}{1+\nu(\partial H(x, y, z, p) / \partial z)}, \\
& \frac{\partial H(x, y, z, p) / \partial y+\partial H(x, y, z, p) / \partial p}{1+\nu(\partial H(x, y, z, p) / \partial z)}
\end{aligned}
$$

are bounded on $\Lambda \times w(\Lambda) \times w^{\Delta}(\Lambda) \times w^{\nabla}(\Lambda)$. If a function $z_{1}(x) \epsilon$ $\mathscr{D}(\Lambda)$ satisfies

$$
\begin{aligned}
& z_{1}^{\Delta \nabla}+H\left(x, z_{1}, z_{1}^{\Delta}, z_{1}^{\nabla}\right) \leq 0, \\
& -z_{1}^{\Delta}(a) \cos \theta+z_{1}(a) \sin \theta \geq \gamma_{1}, \\
& z_{1}^{\nabla}(b) \cos \phi+z_{1}(b) \sin \phi \geq \gamma_{2},
\end{aligned}
$$

and a function $z_{2}(x) \in \mathscr{D}(\Lambda)$ satisfies

$$
\begin{aligned}
& z_{2}^{\Delta \nabla}+H\left(x, z_{2}, z_{2}^{\Delta}, z_{2}^{\nabla}\right) \geq 0, \\
& -z_{2}^{\Delta}(a) \cos \theta+z_{2}(a) \sin \theta \leq \gamma_{1}, \\
& z_{2}^{\nabla}(b) \cos \phi+z_{2}(b) \sin \phi \leq \gamma_{2},
\end{aligned}
$$

then we have

$$
z_{2}(x) \leq u(x) \leq z_{1}(x) .
$$

Remark 88. Theorem 87 implies that the solution of (349), (350) is unique. In fact, if $u_{1}$ and $u_{2}$ are solutions of (349), (350), we just need to let $z_{2}(x)=z_{1}(x)=u_{2}$, and then we obtain $u_{1} \equiv u_{2}$. 


\section{Conflict of Interests}

The authors declare that there is no conflict of interests regarding the publication of this paper.

\section{Authors' Contribution}

All authors contributed equally and significantly to writing this paper. All authors read and approved the final paper.

\section{Acknowledgments}

First, the authors are very grateful to the referees for their careful reading of the paper and the valuable comments and suggestions, which greatly improved it. This work was supported by the National Natural Science Foundation of China (no. 11171286) and by Jiangsu Province Colleges and Universities Graduate Scientific Research Innovative Program (no. CXZZ12-0974).

\section{References}

[1] S. S. Cheng, Partial Difference Equations, Taylor \& Francis, London, UK, 2003.

[2] H. J. Kuo and N. S. Trudinger, "On the discrete maximum principle for parabolic difference operators," Modélisation Mathématique et Analyse Numérique, vol. 27, no. 6, pp. 719-737, 1993.

[3] M. H. Protter and H. F. Weinberger, Maximum Principles in Differential Equations, Prentice-Hall, Englewood Cliffs, NJ, USA, 1967.

[4] M. Renardy and R. C. Rogers, An Introduction to Partial Differential Equations, Springer, Berlin, Germany, 2004.

[5] M. E. Mincsovics and T. L. Horváth, "On the differences of the discrete weak and strong maximum principles for elliptic operators," in Large-Scale Scientific Computing, I. Lirkov, S. Margenov, and J. Waśniewski, Eds., pp. 614-621, Springer, Heidelberg, Germany, 2012.

[6] P. Pucci and J. Serrin, The Maximum Principle, Birkhäuser, Basel, Switzerland, 2007.

[7] P. G. Ciarlet, "Discrete maximum principle for finite-difference operators," Aequationes Mathematicae, vol. 4, pp. 338-352, 1970.

[8] G. Apostolakis and G. F. Dargush, "Variational methods in irreversible thermoelasticity: theoretical developments and minimum principles for the discrete form," Acta Mechanica, vol. 224, no. 9, pp. 2065-2088, 2013.

[9] F. Dong, "Maximum principle and applications of parabolic partial differential equations," IERI Procedia, vol. 3, International Conference on Mechanical and Electronic Engineering, pp. 198-205, 2012.

[10] I. Faragó, S. Korotov, and T. Szabó, “On continuous and discrete maximum principles for elliptic problems with the third boundary condition," Applied Mathematics and Computation, vol. 219, no. 13, pp. 7215-7224, 2013.

[11] J. Karátson and S. Korotov, "Discrete maximum principles for finite element solutions of nonlinear elliptic problems with mixed boundary conditions," Numerische Mathematik, vol. 99, no. 4, pp. 669-698, 2005.

[12] J. Karátson and S. Korotov, "Discrete maximum principles for finite element solutions of some mixed nonlinear elliptic problems using quadratures," Journal of Computational and Applied Mathematics, vol. 192, no. 1, pp. 75-88, 2006.

[13] J. Karátson, S. Korotov, and M. Křížek, "On discrete maximum principles for nonlinear elliptic problems," Mathematics and Computers in Simulation, vol. 76, no. 1-3, pp. 99-108, 2007.

[14] A. Mareno, "Maximum principles and bounds for a class of fourth order nonlinear elliptic equations," Journal of Mathematical Analysis and Applications, vol. 377, no. 2, pp. 495-500, 2011.

[15] P. Pucci and J. Serrin, "The strong maximum principle revisited," Journal of Differential Equations, vol. 196, no. 1, pp. 1-66, 2004.

[16] S. Hilger, "Analysis on measure chains-a unified approach to continuous and discrete calculus," Results in Mathematics, vol. 18, no. 1-2, pp. 18-56, 1990.

[17] R. Agarwal, M. Bohner, D. O’Regan, and A. Peterson, "Dynamic equations on time scales: a survey," Journal of Computational and Applied Mathematics, vol. 141, no. 1-2, pp. 1-26, 2002.

[18] A. Slavík, "Dynamic equations on time scales and generalized ordinary differential equations," Journal of Mathematical Analysis and Applications, vol. 385, no. 1, pp. 534-550, 2012.

[19] B. Jackson, "Partial dynamic equations on time scales," Journal of Computational and Applied Mathematics, vol. 186, no. 2, pp. 391-415, 2006.

[20] C. D. Ahlbrandt and C. Morian, "Partial differential equations on time scales," Journal of Computational and Applied Mathematics, vol. 141, no. 1-2, pp. 35-55, 2002.

[21] M. Bohner and A. Peterson, Dynamic Equations on Time Scales: An Introduction with Applications, Birkhäuser, Boston, Mass, USA, 2001.

[22] M. Bohner and A. Peterson, Advances in Dynamic Equations on Time Scales, Birkhäuser, Boston, Mass, USA, 2003.

[23] N. Martins and D. F. M. Torres, "Calculus of variations on time scales with nabla derivatives," Nonlinear Analysis: Theory, Methods \& Applications, vol. 71, no. 12, pp. e763-e773, 2009.

[24] P. Stehlík, "Maximum principles for elliptic dynamic equations," Mathematical and Computer Modelling, vol. 51, no. 9-10, pp. 1193-1201, 2010.

[25] P. Stehlík and B. Thompson, "Maximum principles for second order dynamic equations on time scales," Journal of Mathematical Analysis and Applications, vol. 331, no. 2, pp. 913-926, 2007. 


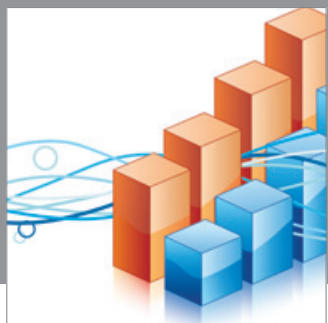

Advances in

Operations Research

mansans

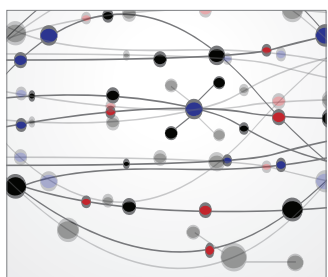

The Scientific World Journal
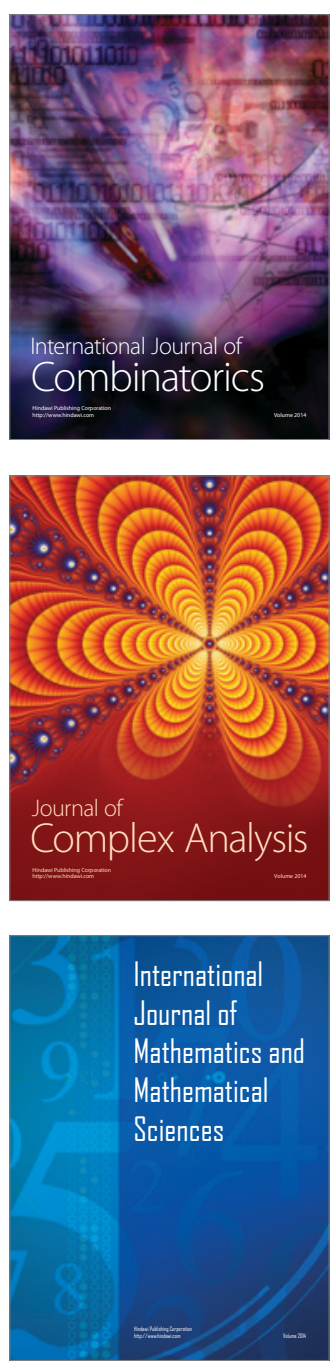
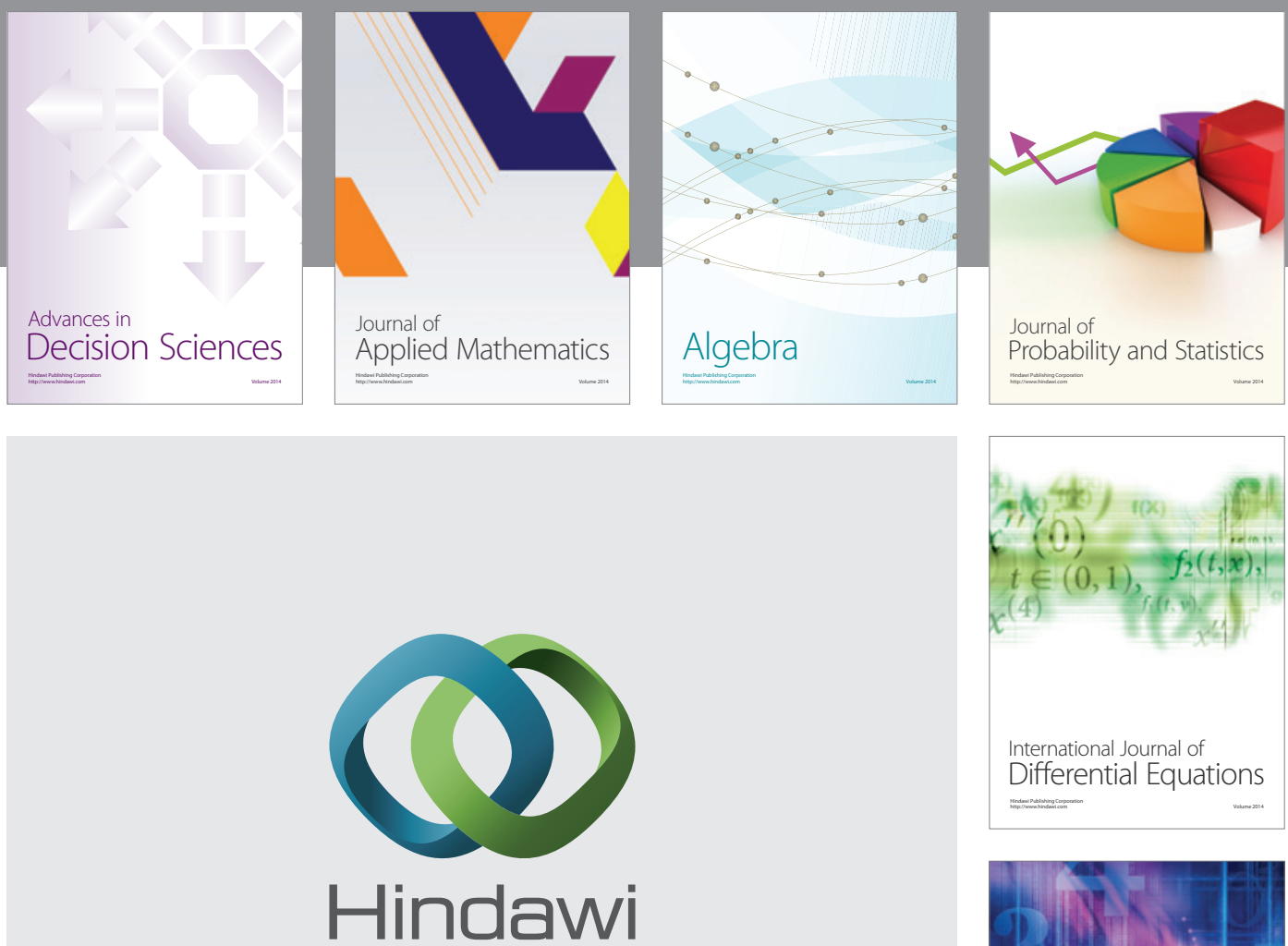

Submit your manuscripts at http://www.hindawi.com
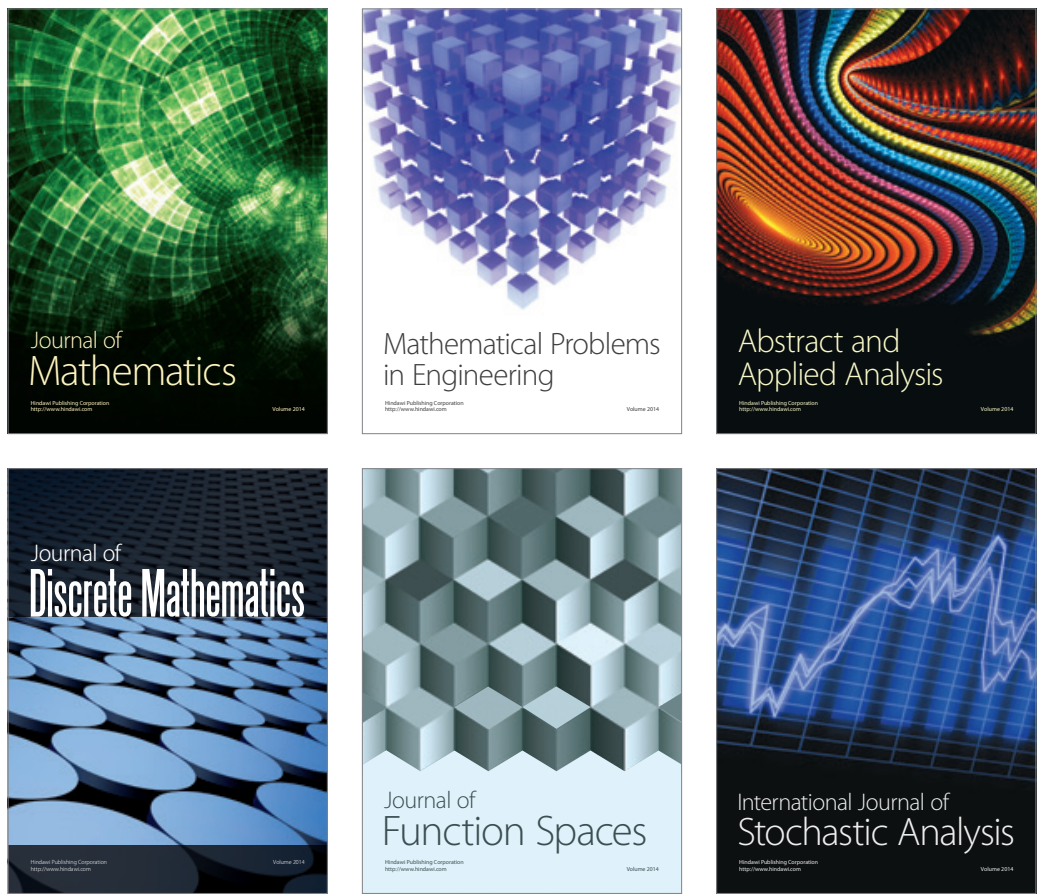

Journal of

Function Spaces

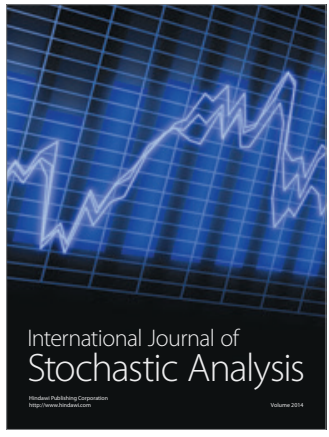

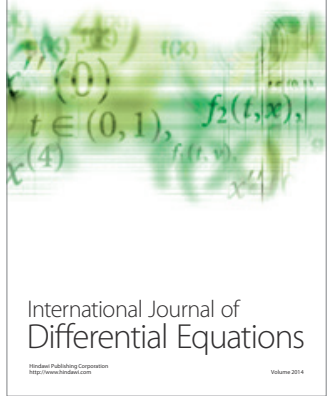
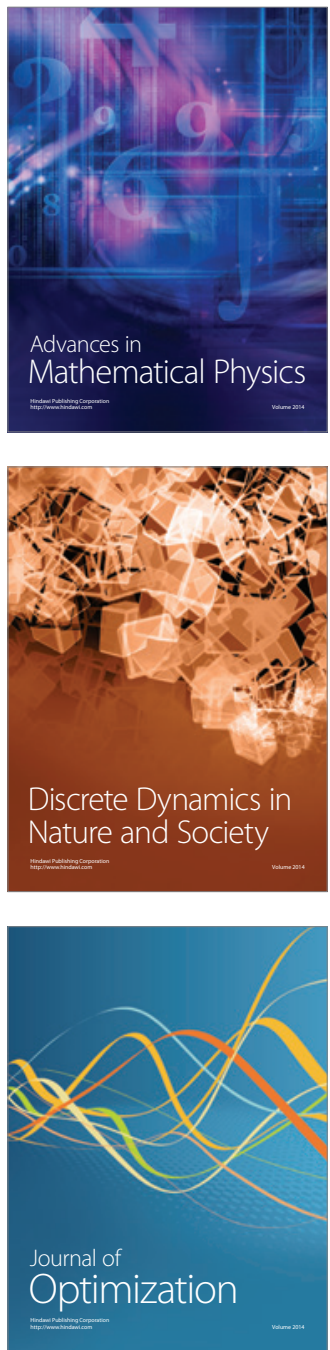